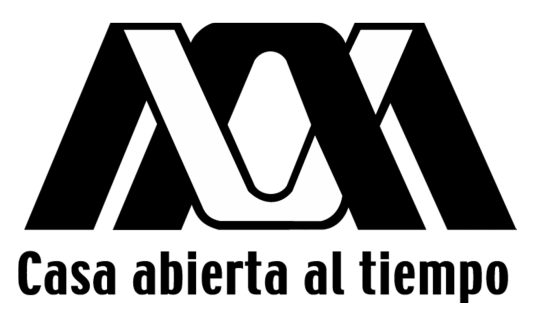

Universidad Autónoma Metropolitana

UNIDAD IZTAPALAPA

\title{
MODELADO DE LA DIFUSIÓN EN EMULSIONES DOBLES
}

TESIS QUE PARA OBTENER EL GRADO DE MAESTRO EN CIENCIAS (INGENIERÍA QUÍMICA)

Presenta:

I.Q.I. Karla María Hernández Verdín

Asesor:

Dr. José de Jesús Álvarez Ramírez 


\section{Agradecimientos}

A Dios por darme la oportunidad de tener un logro más en mi vida.

A mi asesor el Doctor José de Jesús Álvarez Ramírez

A mis sinodales los Doctores Rafael Escalera Pérez y Guillermo Fernández Anaya.

A mis padres (Zenaida, Chony y Gerardo), por estar conmigo, por su apoyo incondicional, por sus palabras de aliento, por creer en mí, pero sobre todo por compartir conmigo este logro, el cual es de ustedes también, los amo.

A mis hermanos (Luis, Fabiola, Cinthia y Zulema) por acompañarme y apoyarme siempre a pesar de la distancia.

A la memoria de mi mami costa porque sin ese ángel que intercede por mí quizá esto no sería posible.

A mis amigos en especial a mi compadre José Pablo, por todos esos momentos en que compartimos risas y quejas, echamos chisme, pero sobre todo por no dejar que tirara la toalla, los aprecio de verdad.

A mi hijo Carlos Giovanny por acompañarme en la elaboración de esta tesis, soportando el ir y venir de la escuela, por ser mi inspiración y mi fuerza, por esas sonrisas y por iluminar mi vida, te amo.

A mi esposo Carlos porque esta tesis tiene mucho de sus conocimientos, por decirme si se puede, cuando yo pensé que no podía, por no dejar que me diera por vencida, pero sobre todo por estar siempre conmigo y ser el pilar más sólido en mi vida, te amo. 


\section{Resumen}

El presente trabajo se plantea la solución numérica del modelo de transferencia de masa de Morales-Zárate et al. (2008) en sistemas de emulsión doble. El modelo se resolvió utilizando el método de diferencias finitas con lenguaje de programación en Matlab. Se hizo un análisis de estabilidad numérica del modelo discretizado. Además, mediante comparación con datos experimentales de la literatura, se hicieron ajustes del modelo y se encontraron los valores de los parámetros del modelo para diferentes sistemas de emulsión doble. Se encontró que el modelo de Morales-Zárate es adecuado para la predicción de la evolución de la concentración. 


\section{Índice general}

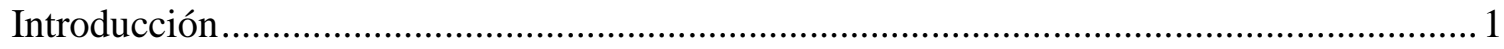

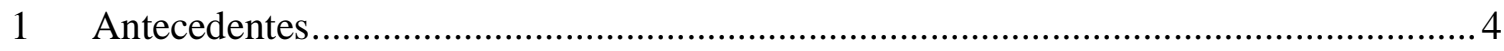

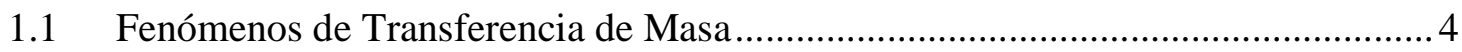

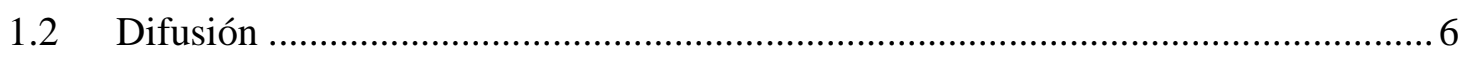

1.3 Ecuaciones de Fick de la difusión....................................................................

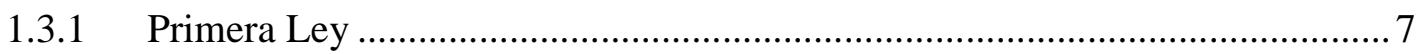

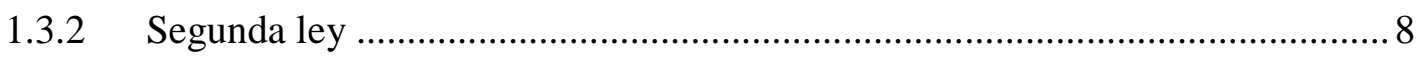

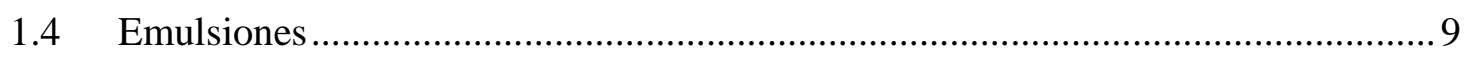

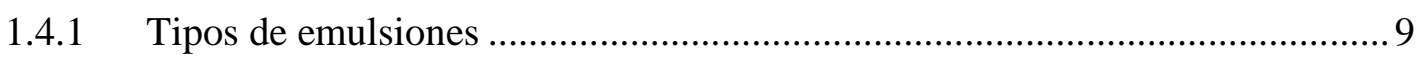

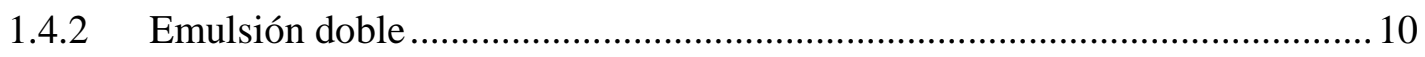

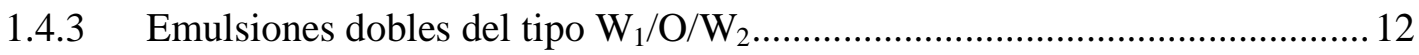

1.5 Solución de ecuaciones diferenciales por métodos numéricos ............................. 14

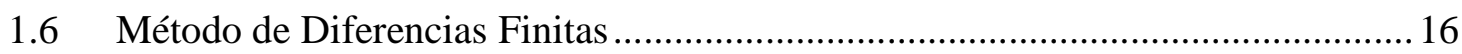

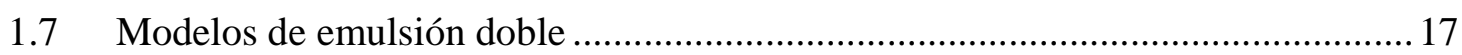

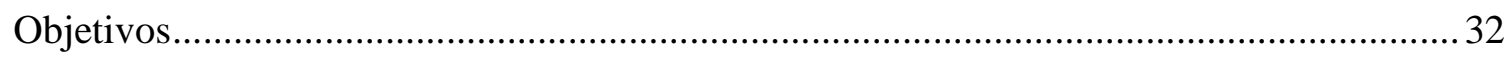

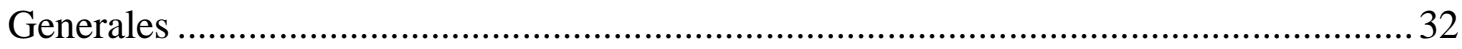

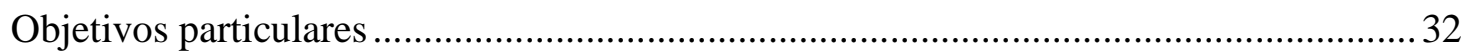

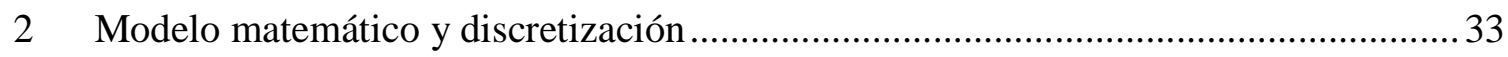

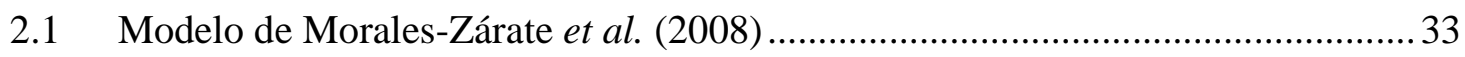

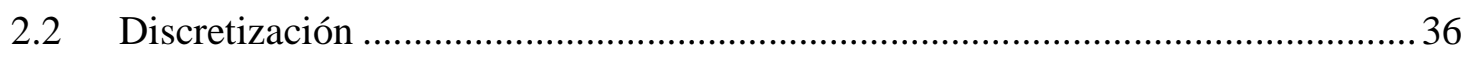

3 Análisis de la estabilidad y consistencia de la solución numérica ................................39

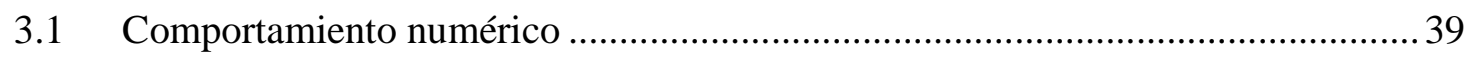

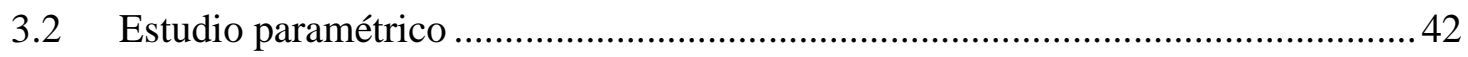

$4 \quad$ Resultados y discusión sobre el ajuste de datos experimentales .................................. 48

Karla María Hernández Verdín $\quad$ Tesis de Maestría 


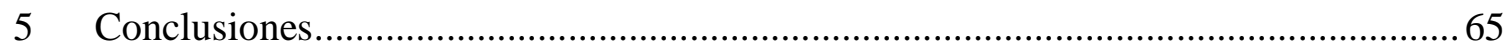

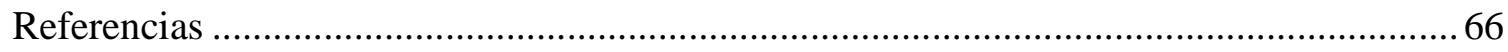

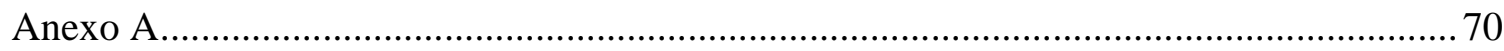

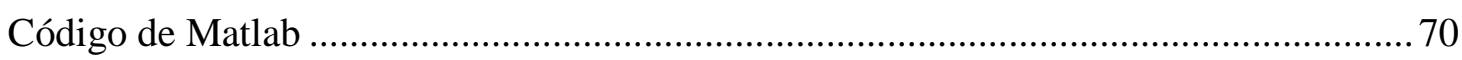

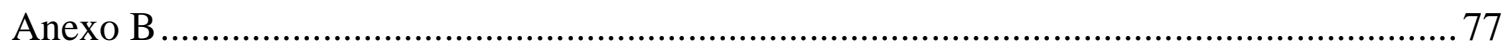

Modelado de la transferencia de masa en la microescala .................................................. 77 


\section{Índice de Figuras}

Figura 1. Planteamiento esquemático de la Ley de Fick. ................................................ 8

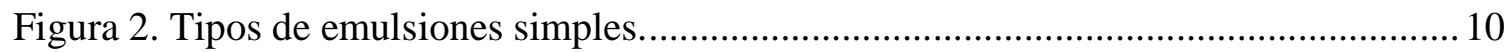

Figura 3. Esquema de una emulsión doble del tipo a) $\mathrm{W}_{1} / \mathrm{O} / \mathrm{W}_{2}$ o b) $\mathrm{O}_{1} / \mathrm{W} / \mathrm{O}_{2} \ldots \ldots \ldots \ldots \ldots \ldots . . . . . . .10$

Figura 4.- Transferencia de masa en emulsiones. (Izquierda) Transferencia de masa en emulsión simple; (Derecha) Transferencia de masa en emulsiones dobles ((Kabalnov, 1994)

Figura 5. Sistema trifásico. Escala I: corresponde al sistema macroscópico; Escala II: representa una muestra de las gotas de membrana líquida rodeada de la fase externa $\gamma$, mientras que la escala III muestra las gotas en la fase de la membrana..............................26

Figura 6. Esquema del proceso de recubrimiento de perlas de hidrogel ..............................2 28

Figura 7. Esquema de un sistema de emulsión doble del tipo W/O/W................................ 33

Figura 8. Evolución de la concentración en la región $\eta$ para diferentes valores de $\gamma$. Aquí se tomó $N_{r}=100, c_{\eta}^{0}=0$ y $B i_{m}=\psi=1$.

Figura 9. Perfiles de concentración en el dominio $\omega$ como función del radio adimensional y tiempo. Se fijó el número de nodos en el tiempo para ser $N_{r} 100, c_{\eta}^{0}=0$ y $B i_{m}=\psi=1 . .40$ Figura 10. Efecto del número de Biot de masa y el parámetro $\psi$ en la relación mínima de tamaños de paso $\gamma$. Aquí se tomó $\mathrm{N}_{\mathrm{r}}=50$ y $c_{\eta}^{0}=0$. 42

Figura 11. Efecto de a) el número de Biot de masa y b) el parámetro $\psi$ en la concentración de soluto no dimensional en el flujo externo. Aquí se usó $\mathrm{N}_{\mathrm{r}}=50$, número de nodos en el tiempo $=1000 \mathrm{~N}_{\mathrm{r}}, c_{\eta}^{0}=0$ y siempre que sea aplicable $\mathrm{Bi}_{\mathrm{m}} \mathrm{o} \psi=1$

Figura 12. Efecto de a) el número de Biot de masa y b) el parámetro $\psi$ en la concentración de soluto no dimensional en el dominio $\omega$. Aquí se usó $\mathrm{N}_{\mathrm{r}}=50$, número de nodos en el tiempo $=1000 \mathrm{~N}_{\mathrm{r}}, c_{\eta}^{0}=0$ y siempre que sea aplicable $\mathrm{Bi}_{\mathrm{m}}$ o $\psi=1$ 45

Figura 13. Tiempo necesario para llegar a la solución de estado estacionario en función de número de Biot de masa y el parámetro $\psi$. Aquí se tomó $c_{\eta}^{0}=0, N_{r}=50$ y $\gamma=5.88 \times 10^{-4}$. 
Figura 14. a) Perfiles de liberación de emulsiones dobles del tipo $\mathrm{W}_{1} / \mathrm{O} / \mathrm{W}_{2}$ con $2 \%$ de $\mathrm{NaCl}, \mathrm{b}$ ) perfil de liberación de $\mathrm{NaCl}$ en la gota interior para diferentes tiempos. 49

Figura 15. a) Perfiles de liberación de emulsiones del tipo $\mathrm{W}_{1} / \mathrm{O} / \mathrm{W}_{2}$ con $4 \%$ de $\mathrm{NaCl}$, b) Perfil de liberación de $\mathrm{NaCl}$ en la gota interior para diferentes tiempos.

Figura 16. a) Perfiles de liberación de emulsión doble del tipo $\mathrm{W}_{1} / \mathrm{O} / \mathrm{W}_{2}$ con $6 \%$ de $\mathrm{NaCl}$, b) Perfil de liberación de $\mathrm{NaCl}$ de la gota interior para diferentes tiempos.

Figura 17. a) Perfil de liberación de emulsión doble del tipo $\mathrm{W}_{1} / \mathrm{O} / \mathrm{W}_{2}$ con $8 \%$ de $\mathrm{NaCl}$, b) Perfil de liberación de $\mathrm{NaCl}$ en la gota interna para diferentes tiempos.

Figura 18. Perfiles de liberación de emulsiones dobles del tipo $\mathrm{W}_{1} / \mathrm{O} / \mathrm{W}_{2}$ con $3 \%$ de gelatina, b) Perfil de liberación de $\mathrm{NaCl}$ de la gota interna para diferentes tiempos. .53

Figura 19. Perfiles de liberación de emulsiones dobles del tipo $\mathrm{W}_{1} / \mathrm{O} / \mathrm{W}_{2}$ con $10 \%$ de gelatina, b) Perfil de liberación de $\mathrm{NaCl}$ para diferentes tiempos. .54

Figura 20. Perfiles de liberación de emulsiones dobles del tipo $\mathrm{W}_{1} / \mathrm{O} / \mathrm{W}_{2}$ con $0 \%$ de gelatina, b) Perfil de liberación de $\mathrm{NaCl}$ de la gota interna para diferentes tiempos .55 Figura 21. a) Perfil de liberación (\%) de $\mathrm{NaCl}$, para una concentración de $4.4 \%$ de $\mathrm{NaCl}$, estabilizada con WPI, b) Perfil de liberación de $\mathrm{NaCl}$ de la partícula interna para diferentes tiempos. .56

Figura 22. Perfil de liberación de $\mathrm{NaCl}$, para una concentración de $1 \%$ de $\mathrm{NaCl}$, estabilizada con WPI, b) Perfil de liberación de $\mathrm{NaCl}$ de la partícula interna para diferentes tiempos.

Figura 23. Perfil de liberación de $\mathrm{NaCl}$, para una concentración de $1.5 \%$ de $\mathrm{NaCl}$, estabilizada con WPI, b) Perfil de liberación de $\mathrm{NaCl}$ de la partícula interna para diferentes tiempos 58

Figura 24. Perfil de liberación de $\mathrm{NaCl}$, para una concentración de $4.4 \%$ de $\mathrm{NaCl}$, estabilizada con WPI, b) Perfil de liberación de $\mathrm{NaCl}$ de la partícula interna para diferentes tiempos

Figura 25. Perfil de liberación de $\mathrm{NaCl}$, para una concentración de $4.4 \%$ de $\mathrm{NaCl}$, estabilizada con WPI/C63, b) Perfil de liberación de $\mathrm{NaCl}$ de la partícula interna para diferentes tiempos. 
Figura 26. Perfil de liberación de $\mathrm{NaCl}$, para una concentración de $4.4 \%$ de $\mathrm{NaCl}$, estabilizada con WPI/U63, b) Perfil de liberación de $\mathrm{NaCl}$ de la partícula interna para

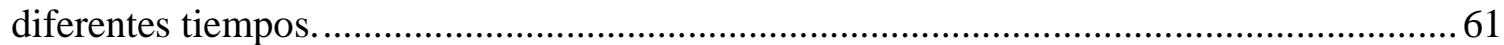

Figura 27. Geometría en 3D para un sistema de emulsión doble........................................... 77

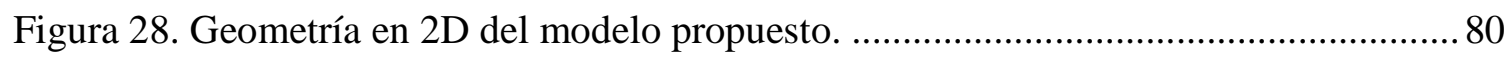

Figura 29. Esquema de liberación de soluto para diferentes tiempos. ................................ 81

Figura 30. Perfiles de concentración en la dirección radial................................................. 82

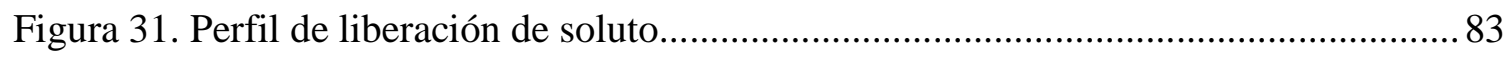




\section{Índice de Tablas}

Tabla 1. Condiciones de frontera para el modelo de Simal et al. (2001) ............................... 31

Tabla 2. Algunas reglas heurísticas para aumentar o disminuir la liberación de soluto........47

Tabla 3. Parámetros estimados y errores calculados. 63 


\section{Nomenclatura}

\section{Letras latinas no negritas}

a

$a_{a}$

$\bar{a}_{a}$

$a_{c}$

$\bar{a}_{c}$

$a_{w}$

$\bar{a}_{w}$

$a_{\eta \omega}$

A

$B i_{m}$

$\mathrm{C}$

$C_{C l}$

$\mathrm{C}_{1}$

$\mathrm{C}_{2}$

$C_{A \gamma}$

$C_{A \mu}$

$C_{A \sigma}$

$C_{B \sigma}$

$C_{e}$

$C_{e}^{*}$

$C_{e q}$
Parámetro de corrección de origen de tiempo [ecuación (58)]

Actividad en la solución (anión)

Actividad en la membrana (anión)

Actividad en la solución (catión)

Actividad en la membrana (catión)

Actividad en la solución (agua)

Actividad en la membrana (agua)

Área interfacial entre la región $\eta$ y la región $\omega$

Área efectiva de la membrana

Número de Biot de masa

Concentración del soluto en la fase membrana

Concentración de iones cloruro en la solución

Concentración de sal en la cámara 1

Concentración de sal en la cámara 2

Concentración local de A en la fase $\gamma$

Concentración local de A en la fase $\mu$

Concentración local de A en la fase $\sigma$

Concentración local de B en la fase $\sigma$

Concentración del soluto en la fase externa

Concentración del soluto en la interfaz de la membrana en la fase externa.

Concentración de equilibrio de $\mathrm{NaCl}$ en la fase continúa.
Adimensional

Adimensional

Adimensional

Adimensional

Adimensional

Adimensional

Adimensional

$\mathrm{m}$

$\mathrm{m}$

Adimensional

$\mathrm{Kmol} / \mathrm{m}^{3}$

$\mathrm{mol} / \mathrm{l}$

$\mathrm{mol} / \mathrm{l}$

$\mathrm{mol} / \mathrm{l}$

$\mathrm{mol} / \mathrm{m}^{3}$

$\mathrm{mol} / \mathrm{m}^{3}$

$\mathrm{mol} / \mathrm{m}^{3}$

$\mathrm{mol} / \mathrm{m}^{3}$

$\mathrm{mol} / \mathrm{dm}^{3}$

$\mathrm{mol} / \mathrm{dm}^{3}$

$\mathrm{mol} / 1$ 


\begin{tabular}{|c|c|c|}
\hline$C_{i}$ & Concentración del soluto en la fase interna & $\mathrm{mol} / 1$ \\
\hline$C_{\eta}$ & Concentración en la región $\eta$ & $\mathrm{mol} / \mathrm{m}^{3}$ \\
\hline$C_{\omega}$ & Concentración del soluto & $\mathrm{mol} / \mathrm{m}^{3}$ \\
\hline$c_{1}^{0}$ & Concentración inicial en la cámara diluida & $\mathrm{mol} / \mathrm{m}^{3}$ \\
\hline$c_{2}^{0}$ & Concentración inicial en la cámara concentrada & $\mathrm{mol} / \mathrm{m}^{3}$ \\
\hline$C_{w}$ & Concentración de agua en la gota de hidrogel. & $\mathrm{mol} / \mathrm{L}$ \\
\hline$C_{w 0}$ & Concentración inicial de agua en la gota & $\mathrm{mol} / \mathrm{L}$ \\
\hline$C_{m}$ & $\begin{array}{l}\text { Concentración del complejo soluto-portador en la } \\
\text { fase de emulsión. }\end{array}$ & $\mathrm{mol} / \mathrm{dm}^{3}$ \\
\hline$C_{m}^{*}$ & $\begin{array}{l}\text { Concentración del complejo soluto-portador en la } \\
\text { interfaz de la fase exterior de la membrana. }\end{array}$ & $\mathrm{mol} / \mathrm{m}^{3}$ \\
\hline$C_{N a}^{m}$ & Concentración de iones de sodio en la membrana & $\mathrm{mol} / \mathrm{l}$ \\
\hline$C_{N a}$ & Concentración de iones sodio en la solución & $\mathrm{mol} / 1$ \\
\hline$C_{\infty}$ & Concentración inicial de agua en la fase de parafina & $\mathrm{mol} / \mathrm{L}$ \\
\hline$C_{R \sigma}$ & Concentración local de $\mathrm{R}$ en la fase $\sigma$ & $\mathrm{mol} / \mathrm{m}^{3}$ \\
\hline$C_{s}=C_{s}^{*}$ & $\begin{array}{l}\text { Concentración equivalente de la especie en } \\
\text { microesferas respecto a la concentración de una } \\
\text { especie en líquido en la gota de la emulsión. }\end{array}$ & $\mathrm{mol} / \mathrm{m}^{3}$ \\
\hline$C_{t}$ & $\begin{array}{l}\text { Concentración de } \mathrm{NaCl} \text { en la fase continua en el } \\
\text { tiempo } \mathrm{t}\end{array}$ & $\mathrm{mol} / \mathrm{m}^{3}$ \\
\hline$D_{A B}$ & Coeficiente de difusión aparente & $m^{2} / s$ \\
\hline$D_{e}$ & $\begin{array}{l}\text { Difusividad efectiva del complejo soluto-portador } \\
\text { en la fase membrana. [ecuación (9)] }\end{array}$ & $m^{2} / s$ \\
\hline$\left.D_{e}\left(\frac{\partial C_{m}}{\partial r}\right)\right|_{r=R}$ & $\begin{array}{l}\text { Flujo de difusión de masa dentro de la interfaz de la } \\
\text { membrana externa. }\end{array}$ & $m^{2} / s$ \\
\hline$D_{e}$ & $\begin{array}{l}\text { Coeficiente de difusión dependiente de la } \\
\text { concentración de } \mathrm{NaCl} \text { de la emulsión interna }\end{array}$ & $m^{2} / s$ \\
\hline
\end{tabular}


[ecuación (14)]

$\begin{array}{lll}D_{e q} & \begin{array}{l}\text { Coeficiente de difusión de NaCl de la emulsión } \\ \text { doble en el equilibrio. }\end{array} & \mathrm{m}^{2} / \mathrm{s} \\ D_{2} \equiv D_{a} & \begin{array}{l}\text { Coeficiente de difusión del fármaco en la fase de la } \\ \text { emulsión. }\end{array} & \mathrm{m}^{2} / \mathrm{s} \\ D_{A \sigma} & \text { Difusividad molecular de la especie A en la fase } \sigma & \mathrm{m}^{2} / \mathrm{s} \\ D_{R \sigma} & \text { Difusividad molecular de la especie Ren la fase } \sigma & \mathrm{m}^{2} / \mathrm{s} \\ D_{A \mu} & \text { Difusividad molecular de la especie A en la fase } \mu & \mathrm{m}^{2} / \mathrm{s} \\ D_{B \sigma} & \text { Difusividad molecular de la especie B en la fase } \sigma & \mathrm{m}^{2} / \mathrm{s} \\ D_{A \gamma} & \text { Difusividad molecular de la especie A en la fase } \gamma & \mathrm{m}^{2} / \mathrm{s} \\ D_{\mathrm{w}} & \text { Coeficiente de difusión efectiva del agua } & \mathrm{m}^{2} / \mathrm{s} \\ D_{\mathrm{s}} & \text { Coeficiente de difusión efectiva del NaCl } & \mathrm{m}^{2} / \mathrm{s} \\ D_{\eta} & \text { Difusividad molecular en la región } \eta & \mathrm{m}^{2} / \mathrm{s} \\ D_{\omega} & \text { Difusividad efectiva } & \mathrm{m}^{2} / \mathrm{s}\end{array}$

Fracción eluída

$\mathrm{h}$

Coeficiente de transferencia de masa externo de las gotas al ambiente

Flujo de sal a través de la membrana

$\mathrm{kg} / \mathrm{s}$

Coeficiente de distribución de $\mathrm{H}_{2} \mathrm{O}$ en las gotas de

K hidrogel y la capa de revestimiento del polímero. [ecuación (51)]

Coeficiente de distribución de equilibrio para el soluto entre la fase $\mu$ y la fase $\gamma$

Coeficiente de distribución de equilibrio para el soluto entre la fase $\mu$ y la fase $\sigma$

$K_{\text {eff }}^{\eta \omega} \quad$ Coeficiente de equilibrio

$K_{\mu} \quad$ Constante de reacción de equilibrio en la fase $\mu$

$k \quad$ Coeficiente de masa externo.

$\mathrm{m} / \mathrm{s}$ 


\begin{tabular}{|c|c|c|}
\hline$k\left(C_{e}-C_{e}^{*}\right)$ & $\begin{array}{l}\text { Flujo de transferencia de masa fuera de la interfaz } \\
\text { de la membrana externa. }\end{array}$ & $\mathrm{kg} / \mathrm{s}$ \\
\hline$k_{f}$ & Tasa de adsorción directa & \\
\hline$k_{r}$ & Tasa de adsorción inversa & \\
\hline$k_{l} \cdot a$ & $\begin{array}{l}\text { Coeficiente volumétrico de transferencia de masa } \\
\text { entre las microesferas y la fase continúa de una } \\
\text { gota. }\end{array}$ & $1 / s$ \\
\hline$k_{\sigma}$ & Constante de velocidad de reacción en la fase $\sigma$ & $\left(m^{3}(\mathrm{~mol} \cdot \mathrm{s})^{-1}\right)$ \\
\hline$k_{\mu}$ & Constante de velocidad de reacción en la fase $\mu$ & $s^{-1}$ \\
\hline$k_{c p}$ & Coeficiente de transferencia de masa & $\mathrm{m} / \mathrm{s}$ \\
\hline$k_{c}$ & Coeficiente de transferencia de masa & $\left(\mathrm{kg}\right.$ de agua $\left./ m^{2} \cdot s\right)$ \\
\hline $\mathrm{m}$ & $\begin{array}{l}\text { Coeficiente de distribución de extracción en la } \\
\text { interfaz de la fase externa de la membrana. }\end{array}$ & \\
\hline $\mathrm{M}_{\mathrm{W}}$ & Peso molecular del agua & $18 \mathrm{~kg} / \mathrm{kmol}$ \\
\hline $\mathrm{N}$ & Número total de glóbulos de la emulsión & \\
\hline $\mathrm{P}_{\mathrm{ms}}$ & Coeficiente de permeabilidad integral del sistema & $\mathrm{m} / \mathrm{s}$ \\
\hline $\mathrm{P}_{\mathrm{S}}$ & Presión de vapor & atm \\
\hline$P_{\mu \sigma}$ & Permeabilidad de la membrana en la interface $\mu$ & $\mathrm{m} / \mathrm{s}$ \\
\hline$P_{\eta \mu}$ & Permeabilidad de la membrana en la interface $\gamma$ & $\mathrm{m} / \mathrm{s}$ \\
\hline$P_{e f f}^{\eta}$ & Permeabilidad efectiva en la región $\eta$ & $\mathrm{m} / \mathrm{s}$ \\
\hline q & $\begin{array}{l}\text { Coeficiente de distribución de extracción en la } \\
\text { interfase de la membrana en la fase interna. }\end{array}$ & \\
\hline $\mathrm{R}$ & Radio de los glóbulos. & $\mathrm{m}$ \\
\hline $\mathrm{R}$ & Constante de los gases [ecuación (63)] & $0.082 \mathrm{~atm} \cdot \mathrm{m}^{3} / \mathrm{kmol} \cdot \mathrm{K}$ \\
\hline$R_{x}$ & $\begin{array}{l}\text { Velocidad de desaparición del complejo soluto- } \\
\text { portador en la fase membrana en la fase interna por } \\
\text { extracción. }\end{array}$ & $\mathrm{mol} / \mathrm{dm}^{3} / \mathrm{s}$ \\
\hline
\end{tabular}




\begin{tabular}{|c|c|c|}
\hline$R_{e q}$ & Tamaño del glóbulo en equilibrio. & $\mathrm{m}$ \\
\hline$R_{\sigma}$ & Velocidad de reacción local en la fase $\sigma$ & $\left(\operatorname{mol}\left(s \cdot m^{3}\right)^{-1}\right)$ \\
\hline$R_{\mu}$ & Velocidad de reacción local en la fase $\mu$ & $\left(\operatorname{mol}\left(s \cdot m^{3}\right)^{-1}\right)$ \\
\hline$R_{\omega}$ & Velocidad de reacción local en la fase $\omega$ & $\left(m o l\left(s \cdot m^{3}\right)^{-1}\right)$ \\
\hline $\mathrm{r}$ & Coordenada radial [ecuación (75)] & \\
\hline $\mathrm{r}$ & $\begin{array}{l}\text { Radio interior en el glóbulo de la emulsión } \\
\text { [ecuación (9)] }\end{array}$ & $\mathrm{m}$ \\
\hline $\mathrm{r}$ & $\begin{array}{l}\text { Coordenada radial discretizada a lo largo del radio } \\
\text { del depósito [ecuación (14)] }\end{array}$ & \\
\hline$r_{0}$ & Radio del volumen promedio & $\mathrm{m}$ \\
\hline$S$ & Concentración local del $\mathrm{NaCl}$ & $k g \mathrm{NaCl} / \mathrm{kg}$ de agua \\
\hline $\mathrm{s}$ & $\begin{array}{l}\text { Concentración del coloide adsorbida } \\
\text { reversiblemente sobre superficies sólidas. }\end{array}$ & \\
\hline $\mathrm{T}$ & Temperatura [ecuación (63)] & $\mathrm{K}$ \\
\hline $\mathrm{t}$ & Tiempo & $\mathrm{s}$ \\
\hline $\mathrm{V}$ & Volumen de cada cámara & $d m^{3}$ \\
\hline$V_{a}$ & Volumen molar parcial (anión) & $d m^{3}$ \\
\hline$V_{c}$ & Volumen molar parcial (catión) & $d m^{3}$ \\
\hline$V_{e}$ & Volumen de la fase externa & $d m^{3}$ \\
\hline$V_{m}$ & Volumen de la fase membrana & $d m^{3}$ \\
\hline$V_{i}$ & Volumen de la fase interna & $d m^{3}$ \\
\hline $\mathrm{W}$ & Concentración de humedad local & $k g$ de agua $/ k g$ inicial \\
\hline
\end{tabular}

\section{Letras latinas negritas}

$\mathbf{D}_{\omega}$

Tensor de difusividad efectiva en la región

$\omega$ 
Vector normal unitario que apunta desde la

$\mathbf{n}_{\mu \sigma}$

región $\mu$ a la región $\sigma$

Vector normal unitario que apunta desde la región $\sigma$ a la región $\mu$

Vector normal unitario que apunta desde la

$\mathbf{n}_{\mu \gamma}$ región $\mu$ a la región $\gamma$

Vector normal unitario que apunta desde la región $\gamma$ a la región $\mu$

Vector normal unitario que apunta desde la región $\omega$ a la región $\eta$

\section{Símbolos griegos}

$\beta$

Parámetro de escala que relaciona la difusividad inicial y de equilibrio.

Adimensional

$\theta \quad$ Porosidad

$\rho_{b} \quad$ Densidad del coloide

$\mathrm{kg} / \mathrm{m}^{3}$

$\lambda$

Coeficiente de filtración

$\varphi$

Fracción de volumen de microesferas en la gota de la emulsión.

$\varepsilon_{\mu \omega}$

Fracción volumen de la fase membrana continua dispersa dentro de la región $\omega$

Adimensional

Fracción volumen de las gotas pequeñas

$\varepsilon_{\sigma \omega}$ dentro de la región $\omega$

Adimensional 


\section{Introducción}

Existen numerosos ejemplos cotidianos de transporte de materia: la difusión de humo y otros contaminantes en la atmósfera; la transferencia de soluto entre las fases de un absorbedor de gas, un extractor o una torre de enfriamiento; el secado de la ropa (difusión del vapor de agua en el aire); el intercambio de oxígeno-gas carbónico en los pulmones.

Así como en el transporte de calor, el transporte de masa puede ocurrir tanto por difusión como por convección, está última representa el transporte de masa que resulta del movimiento global del fluido y la primera el transporte debido a gradientes de concentración.

El transporte convectivo de masa consiste de dos tipos: convección forzada, en la que el movimiento es generada por una fuerza externa, y convección libre, un efecto de flotación en el cual el movimiento global se desarrolla naturalmente como consecuencia de cambios de densidad originados en las diferencias de concentración del medio.

La transferencia de masa por difusión tiene gran importancia en muchos procesos químicos y biológicos. Los modelos matemáticos que describen estos procesos de difusión en la mayoría de los casos son de gran complejidad y su solución analítica no siempre se encuentra disponible, por lo que es necesario utilizar técnicas numéricas para obtener los perfiles de concentración de interés.

Los modelos de difusión son planteados con ayuda de herramientas matemáticas que permiten la descripción de los procesos difusivos, estas herramientas son las leyes de conservación y las ecuaciones diferenciales ordinarias y parciales. Algunos procesos biológicos descritos a partir del modelo de difusión pueden encontrase en (EdelsteinKeshet, 1988; Murray, 1991; Okubo, 1980).

Cuando en un sistema termodinámico multicomponente hay un gradiente de concentraciones, se origina un flujo irreversible de materia, desde las altas concentraciones a las bajas. A este flujo se le llama difusión. La difusión tiende a devolver al sistema a su estado de equilibrio, de concentración constante. 
La difusión es más compleja que el flujo viscoso o la transmisión de calor debido a la innovación de tener que operar con mezclas. En una mezcla que difunde las velocidades de los componentes individuales son distintas y existen varios métodos adecuados para promediar las velocidades de los componentes con el fin de obtener la velocidad local de la mezcla. La elección de esta velocidad local es necesaria para poder definir las velocidades de difusión (Betancourt-Grajales, 1991).

En el análisis de fenómenos de transporte continuamente aparecen ecuaciones cuya resolución analítica es prácticamente imposible debido a la cantidad de operaciones que involucran; es por esto que la investigación científica y tecnológica en esta rama es fundamentalmente impulsada por la adecuada selección de una herramienta de cómputo cuya implementación permita la interpretación de dicho fenómeno.

En ingeniería, muchos problemas se resuelven utilizando ecuaciones diferenciales, pero existe el inconveniente que no todos los problemas pueden ser resueltos analíticamente, por su complejidad, es por esto que se emplean métodos numéricos para encontrar una aproximación a la solución.

Una de las ventajas de utilizar métodos numéricos es que se pueden programar en una computadora de tal manera que sea ésta la que haga la mayoría del trabajo (es decir, los cálculos necesarios para obtener la solución del problema). Esto hace que el trabajo del ingeniero o estudiante se limite solamente al análisis de los resultados, y obviamente preparar el problema para introducir los datos al programa que utilizará en la computadora para resolver el problema.

En este trabajo se propone y evalúa un modelo matemático para la descripción del fenómeno de difusión de un soluto conocido $(\mathrm{NaCl})$ en un sistema de emulsión doble. Esto con el propósito de compararse los resultados obtenidos al resolverse el modelo con datos experimentales encontrados en la literatura.

El modelo hace referencia al trabajo de Morales-Zárate et al. (2008), utilizando las ecuaciones que regulan la transferencia de masa difusiva en coordenadas esféricas. La discretización del modelo se realiza mediante el método de diferencias finitas utilizando un método explícito, y para la solución de dichas ecuaciones se utiliza el lenguaje de 
programación MATLAB. Previo a esto, el modelo matemático se resolvió con el método de diferencias finitas utilizando un método explícito. Al modelo discretizado se le evaluó la consistencia y estabilidad numérica en función de parámetros pertinentes.

Para encontrar la mejor comparación entre las predicciones teóricas y datos experimentales de la literatura, se implementó un procedimiento numérico para minimizar el error. De esta forma, se reportan los valores de los parámetros de transporte contenidos en el modelo de transporte de masa para varios sistemas de emulsión doble publicados. 


\section{CAPÍTULO 1}

\section{Antecedentes}

En este capítulo se presenta la revisión de los trabajos más relevantes relacionados con la investigación. El tema principal a revisar se enfoca en las distintas propuestas y desarrollos sobre los modelos matemáticos aplicados a la solución numérica de emulsiones dobles.

\subsection{Fenómenos de Transferencia de Masa}

Existen en la naturaleza algunos procesos físicos, llamados Fenómenos de Transporte, que involucran transferencia de materia, energía o momentum. Ejemplos de estos procesos son la viscosidad, propagación del calor y la difusión de masa.

Los fenómenos de transporte (Bird et al., 1987) tienen lugar en aquellos procesos conocidos como de transferencia, en los que se establece el movimiento de una propiedad en una o varias direcciones bajo la acción de una fuerza impulsora. Los procesos de transferencia de masa son importantes ya que la mayoría de los procesos químicos requieren de la purificación inicial de las materias primas o de la separación final de productos y subproductos.

La transferencia de masa juega un papel fundamental en la industria. Muchas son las aplicaciones que industrialmente están gobernadas por los principios de transferencia de materia. Algunos de los más importantes son: Destilación, extracción liquido-liquido secado, absorción, adsorción y humidificación.

Debe distinguirse entre la transferencia de masa y el movimiento de masas de fluido (o flujo de fluidos) que se presenta en un nivel macroscópico conforme un fluido se transporta de un lugar a otro. Cuando existen diferencias de concentraciones en una mezcla, se produce una migración selectiva de los componentes de la mezcla cuya distribución de concentraciones no sea uniforme. Este movimiento se da específicamente desde las zonas de alta concentración hasta las zonas de baja concentración (de la misma forma como en transferencia de calor el flujo se da desde la zona de alta temperatura hacia la zona de baja temperatura) y esto es lo que en fenómenos de transporte se conoce como transferencia de masa. 
La fuerza impulsora primaria para el flujo de fluidos es la diferencia de presión, en tanto que, para la transferencia de masa, es la diferencia de concentración (Yunus y Afshin, 2011).

La transferencia de masa cambia la composición de soluciones y mezclas mediante métodos que no implican necesariamente reacciones químicas (Salvadori y Baron, 1992) y se caracteriza por transferir una sustancia a través de una u otra escala molecular. Cuando se ponen en contacto dos fases que tienen diferente composición, la sustancia que se difunde abandona un lugar de una región de alta concentración y pasa a un lugar de baja concentración. La velocidad de transferencia de masa depende de una fuerza impulsora (diferencia de concentración) sobre una resistencia, que indica la dificultad de las moléculas para transferirse en el medio. Existen dos modos de transferencia de masa:

- Molecular: la masa se transfiere por medio del movimiento molecular fortuito en los fluidos debido a una diferencia de concentraciones. La difusión molecular puede ocurrir en sistemas de fluidos estancados o en fluidos que se están moviendo.

- Convectiva: la masa se transfiere debido al movimiento global del fluido. Puede ocurrir que el movimiento se efectúe en régimen laminar o turbulento. El flujo turbulento resulta del movimiento de grandes grupos de moléculas y es influenciado por las características dinámicas del flujo. Tales como densidad, viscosidad, etc.

Usualmente, ambos mecanismos actúan simultáneamente. Sin embargo, uno puede ser cuantitativamente dominante y por lo tanto, para el análisis de un problema en particular, es necesario considerar solo a dicho mecanismo.

La rapidez con la cual un componente se transfiere de una fase a otra depende del coeficiente de transferencia de masa. El fenómeno de difusión molecular conduce finalmente a una concentración completamente uniforme de sustancias a través de una solución que inicialmente no era uniforme. La transferencia se termina cuando se alcanza el equilibrio.

Los coeficientes de transferencia de masa tienen mucha importancia, pues al regular la rapidez con la cual se alcanza el equilibrio, controlan el tiempo que se necesita para la difusión. Los coeficientes de rapidez para los diferentes componentes en una fase dada difieren entre sí en mayor grado bajo condiciones en donde prevalece la difusión molecular. 
En condiciones de turbulencia, en que la difusión molecular carece relativamente de importancia, los coeficientes de transferencia se vuelven más parecidos para todos los componentes.

\subsection{Difusión}

La difusión es un proceso físico irreversible, en el que partículas materiales se introducen en un medio que inicialmente estaba ausente, aumentando la entropía del sistema conjunto formado por las partículas difundidas y el medio donde se difunden.

Cuando en un sistema termodinámico multicomponente hay un gradiente de concentraciones, se origina un flujo irreversible de materia, desde las altas concentraciones a las bajas.

La difusión aparece como consecuencia de la no-existencia de equilibrio químico; tiende a devolver al sistema a su estado de equilibrio de concentración constante.

Normalmente los procesos de difusión están sujetos a la ley de Fick. La membrana permeable puede permitir el paso de partículas y disolvente siempre a favor del gradiente de concentración. La difusión, proceso que no requiere aporte energético, es frecuente como forma de intercambio celular.

La difusión se produce por dos mecanismos básicos: difusión molecular o Browniana y difusión turbulenta. La primera, es aquella en la cual las partículas suspendidas en un fluido, experimentan un movimiento aleatorio, producido por colisiones entre ellas; este fenómeno se conoce como movimiento browniano. La segunda, es la difusión que incluye procesos inducidos por agentes externos al fluido, los cuales, entregando energía de alguna forma al fluido, lo vuelven homogéneo, esto hace que en la difusión turbulenta las partículas se difundan más rápidamente que en la difusión molecular.

Como no todos los procesos de difusión ocurren a la misma velocidad, se ha definido un número llamado Coeficiente de difusión, usualmente denotado por $\mathrm{D}$, que mide la velocidad de la difusión de una sustancia en otra. 
Las leyes que rigen los procesos de difusión de materia o masa son: la ley de conservación de masa (enunciada por Lavoiser en el siglo XIX) y la ley de Fick (enunciada por Fick en $1855)$.

La ley de conservación de materia, afirma que: la tasa de variación de la concentración de partículas en una región, en un instante dado, es igual a la tasa de entrada de las partículas, menos la tasa de salida de las partículas, en la región considerada.

La ley de conservación de masa expresada en términos matemáticos es:

$$
\frac{\partial C(x, t)}{\partial t}=-\frac{\partial J(x, t)}{\partial x}
$$

\subsection{Ecuaciones de Fick de la difusión}

\subsubsection{Primera Ley}

La primera ley de Fick fue deducida por Fick en 1855, por analogía con la ley de Fourier sobre la conducción de calor, pero con la importante diferencia de que la transferencia de materia, contrariamente al calor, mantiene a todo el fluido en movimiento, excepto en circunstancias especiales en las que los componentes se mueven por igual en todas direcciones.

Se utiliza en estado estacionario, es decir, cuando la concentración dentro del volumen de la difusión no cambia con respecto al tiempo.

Establece que el flujo difusivo que atraviesa una superficie $(\mathrm{J})$ es directamente proporcional al gradiente de concentración. Si se considera la difusión unidimensional de una sustancia y se supone que la concentración varía con la posición a lo largo del eje x y se denomina $\mathrm{J}$ a la densidad de corriente de partículas es decir, al número efectivo de partículas que atraviesan por unidad de tiempo un área unitaria perpendicular a la dirección en la que tiene lugar la difusión. La ley de Fick se puede enunciar:

$$
J=-D \frac{\partial C}{\partial x}
$$


El signo negativo hace hincapié en que la difusión ocurre en el sentido del decremento en la concentración, y el gradiente es negativo pero el flujo de masa debe ser positivo. La difusividad es una característica de un componente y su entorno (temperatura, presión, concentración; ya sea en solución líquida, gaseosa o sólida y la naturaleza de los otros componentes). Obsérvese la representación esquemática de la Ley de Fick en la Figura 1.

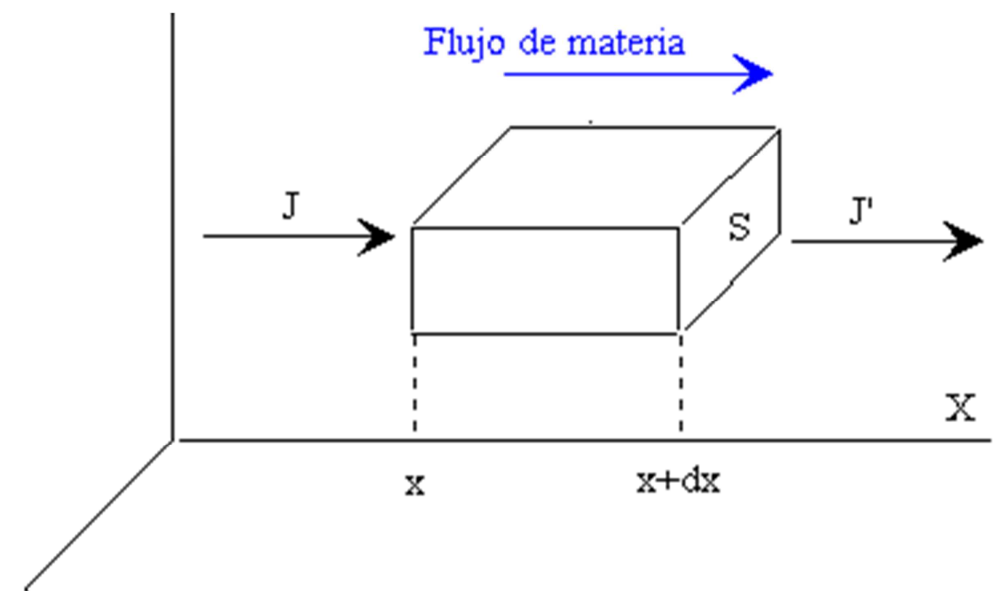

Figura 1. Planteamiento esquemático de la Ley de Fick.

\subsubsection{Segunda ley}

Al derivar la ecuación (1) con respecto a x y reemplazando en la ecuación (2), se obtiene:

$$
\frac{\partial C(x, t)}{\partial t}=D \frac{\partial^{2} C(x, t)}{\partial x^{2}}
$$

La ecuación (3) es llamada la segunda ley de Fick o Ley de Difusión Unidimensional.

La segunda ley de Fick se utiliza en la difusión no-constante, es decir cuando la difusión es en estado no estacionario, en los que el coeficiente de difusión es independiente del tiempo.

Esta ley establece que la velocidad de cambio de la composición de la muestra es igual al coeficiente de difusión por la velocidad de cambio del gradiente de concentración. La solución de la segunda ley de Fick es:

$$
\frac{C_{s}-C_{x}}{C_{s}-C_{0}}=\operatorname{erf}\left(\frac{x}{2 \sqrt{D t}}\right)
$$


Dónde:

$\mathrm{C}_{\mathrm{s}}=$ Concentración superficial del elemento en el gas que difunde dentro de la superficie.

$\mathrm{C}_{0}=$ Concentración inicial uniforme del elemento en el sólido .

$\mathrm{C}_{\mathrm{x}}=$ Concentración del elemento a la distancia $\mathrm{x}$ de la superficie en el tiempo $\mathrm{t}$.

$\mathrm{x}=$ Distancia desde la superficie

$\mathrm{D}=$ Coeficiente de difusión del elemento o soluto que difunde .

$\mathrm{t}=$ tiempo.

\subsection{Emulsiones}

Una emulsión es un sistema que contiene dos fases líquidas inmiscibles, una de las cuales está dispersada en la otra, y cuya estructura es estabilizada por una agente surfactante conocido también como emulsionante. La noción de estabilidad es por supuesto relativa, pero se refiere a una casi ausencia de cambio durante un período de tiempo suficientemente largo para el propósito de la aplicación práctica, lo cual puede variar de algunos minutos a algunos años (Salager, 1999).

\subsubsection{Tipos de emulsiones}

La primera clasificación fue propuesta por W. Ostwald, y se basa en la distribución que se hace de acuerdo a la naturaleza de sus fases (continua y dispersa), las cuales pueden ser acuosa (W: agua) u oleosa (O: aceite). El término acuoso se refiere a cualquier líquido hidrofílico, altamente polar, y oleoso a cualquier líquido hidrofóbico o no polar (Becher, 1972).

Se tiene entonces que las emulsiones pueden clasificarse en dos tipos generales (Figura 2):

- Emulsiones W/O: Dónde la fase continua es aceite y la fase dispersa es agua.

- Emulsiones $\mathrm{O} / \mathrm{W}$ : la fase continua es agua y la fase dispersa es aceite. 

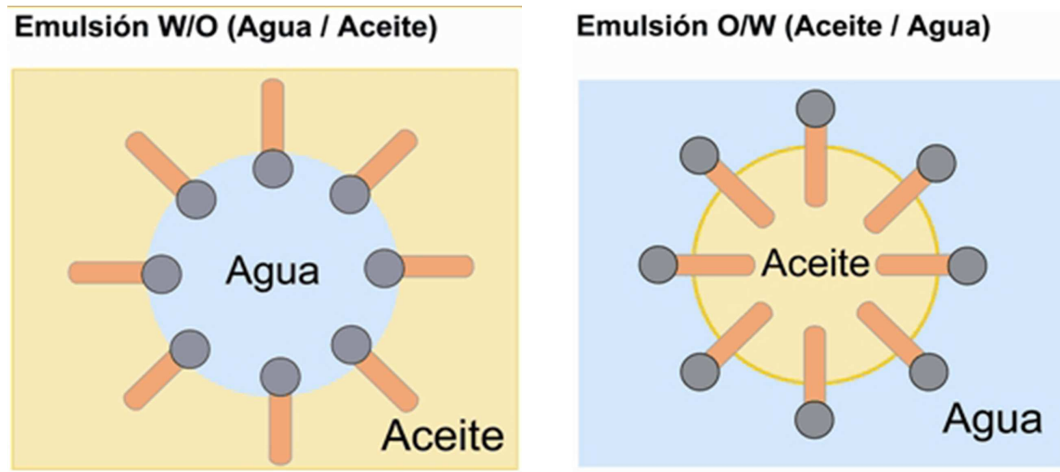

Figura 2. Tipos de emulsiones simples.

\subsubsection{Emulsión doble}

Las emulsiones múltiples o emulsiones de emulsiones, son también conocidas como membranas líquidas emulsionadas, descubiertas por Seifritz (1925) son sistemas dispersos complejos en los que la fase dispersa es una emulsión y están caracterizadas por tener una baja estabilidad termodinámica. Cada uno de los glóbulos en estos sistemas contiene uno o más compartimientos de una fase, la cual está separada de una fase químicamente similar, a través de una membrana de una fase diferente e inmiscible, es por ello que también suele dárseles el nombre de membranas dobles. Igual que las emulsiones simples, se pueden clasificar según la naturaleza de las fases, de modo que los dos tipos más comunes de estos sistemas son las emulsiones agua-en-aceite-en-agua $\left(\mathrm{W}_{1} / \mathrm{O} / \mathrm{W}_{2}\right)$ o las aceite-agua-en-aceite $\left(\mathrm{O}_{1} / \mathrm{W} / \mathrm{O}_{2}\right)$ (Muschiolik, 2007), se utilizan los números 1 y 2 para indicar que la fase externa continua y la interna, si bien son miscibles, pueden tener composiciones distintas (ver Figura 3).
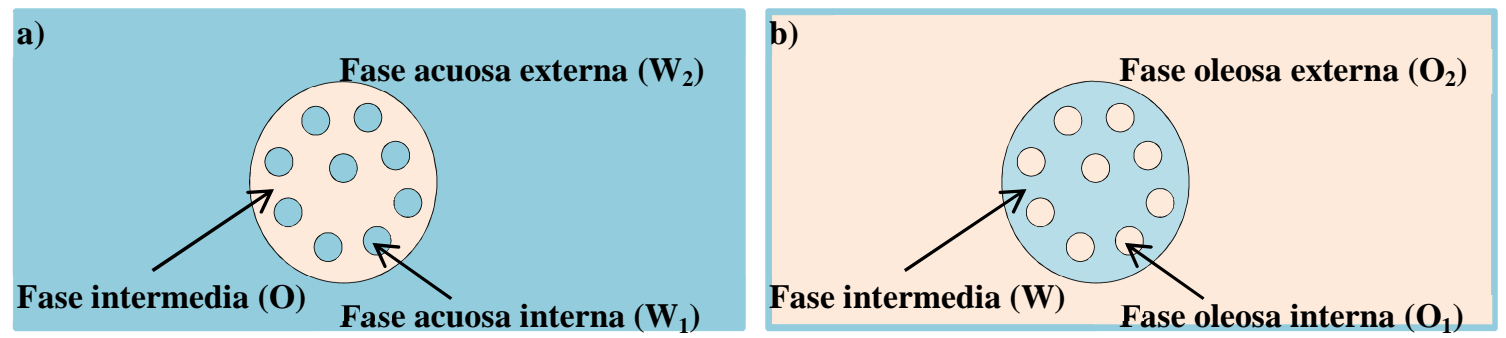

Figura 3. Esquema de una emulsión doble del tipo a) $W_{1} / O / W_{2}$ o b) $O_{1} / W / O_{2}$. 
En ambos casos puede verse que existe una fase líquida que separa dos fases líquidas miscibles entre sí, esta fase líquida inmiscible en las otras dos que puede actuar como una membrana y es lo que se conoce con el nombre de membrana líquida emulsionada (ELM por sus siglas en inglés).

La comparación de las ELM con otro tipo de membranas, muestra una serie de ventajas competitivas, entre ellas:

- La gran superficie de transferencia de masa.

- Menor resistencia a la transferencia de masa en comparación a la resistencia a la difusión que ofrecen ciertos polímeros sólidos, debido al reducido espesor de la fase membrana y la poca resistencia a la difusión que ofrecen los líquidos.

- Alta selectividad. Las ELM pueden actuar como sustrato muy selectivo debido a la utilización de transportadores y a la modificación de ciertos parámetros como el $\mathrm{pH}$.

- La relación de la fase membrana a la fase externa es generalmente muy baja, de allí que se requieren pequeñas cantidades de solvente y agente transportador, cuyos costos son generalmente elevados.

La estructura de las emulsiones dobles permite la posibilidad de encapsular sustancias de diferente naturaleza tanto en la fase interna como en la intermedia (Raynal et al., 1993) protegiéndolas de agentes externos y a su vez pudiendo controlar su liberación mediante estímulos (temperatura, $\mathrm{pH}$, entre otros). Es por este motivo que su estudio en campos como el farmacéutico y cosmético entre otros es de gran interés, pudiéndose formular un sistema de encapsulación con una liberación controlada en el tiempo y localizada.

Ya que las emulsiones dobles son sistemas complicados, generalizar los resultados experimentales relativos a la transferencia de masa en dichos sistemas es una tarea difícil (Wronski et al., 2012). Por lo tanto, parece ser razonable considerar un modelo matemático que podría hacer más fácil la evaluación preliminar de la influencia de determinados parámetros en el proceso de liberación de las microesferas internas. En general, los modelos se basan en la segunda ley de Fick, introduciendo el coeficiente de difusión efectiva que se refiere al volumen de la gota de emulsión que contiene las microesferas. 
Estos planteamientos no describen el mecanismo real de la transferencia de masa en estos sistemas.

La transferencia de masa en emulsiones no sólo se dirige por la diferencia en la curvatura de las gotas, sino también por la diferencia en sus composiciones. Esto se observa cuando dos aceites químicamente diferentes son emulsificados por separado y las emulsiones resultantes son mezcladas. La maduración de composición o composición "ripening" involucra el intercambio de moléculas de aceite entre gotas de emulsiones con diferentes composiciones ya que este término se utiliza cuando la composición de las gotas no es uniforme y los gradientes de concentración dirigen el intercambio de masa, por tanto inducen cambios en el tamaño de la gota (Kabalnov, 1994).

La fuerza termodinámica que dirige este mecanismo es la diferencia en el potencial químico de las moléculas de aceite en los diferentes entornos.

La transferencia de masa de una emulsión a otra es controlada por la entropía de la mezcla y procederá hasta que la composición de las gotas sea idéntica (Figura 4).

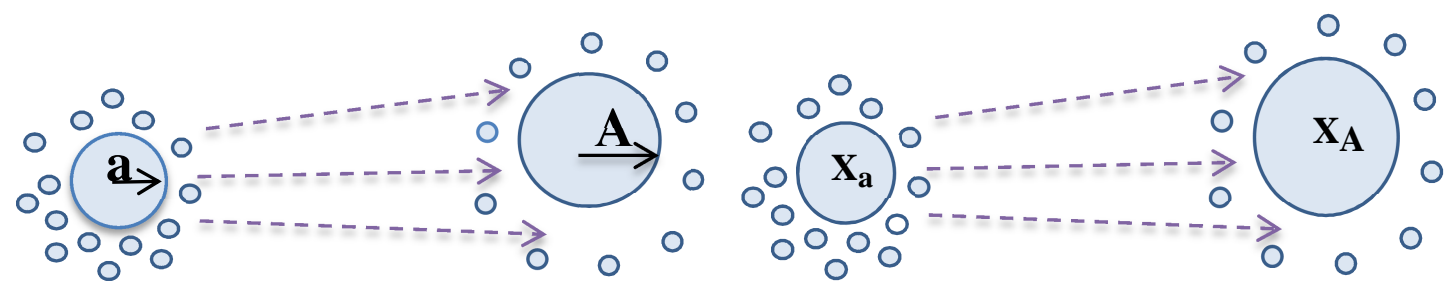

Figura 4.- Transferencia de masa en emulsiones. (Izquierda) Transferencia de masa en emulsión simple; (Derecha) Transferencia de masa en emulsiones dobles ((Kabalnov, 1994).

\subsubsection{Emulsiones dobles del tipo $\mathrm{W}_{1} / \mathrm{O} / \mathrm{W}_{2}$}

Las emulsiones dobles del tipo $\mathrm{W}_{1} / \mathrm{O} / \mathrm{W}_{2}$ consisten en gotas de agua dispersas en grandes glóbulos de aceite los cuales están dispersos a su vez en una fase acuosa continua.

Las emulsiones dobles del tipo $\mathrm{W}_{1} / \mathrm{O} / \mathrm{W}_{2}$ son las más usadas y pueden tener varias aplicaciones: 
- Separaciones: en estos procesos una o varias especies son transportadas de la fase externa hacia la face interna de la emulsión doble.

En el campo de la Ingeniería Química, los primeros trabajos en los que se emplearon emulsiones dobles se realizaron en la campaña Exxon a finales de la década de los sesenta y princio de los setenta, y consistieron en la separación de mezclas de hidrocarburos (alcanos y tolueno o alcanos y benceno) (Cárdenas, 1995).

- Liberación controlada (Cárdenas, 1995): En ésta aplicación, el flujo de compuesto va en sentido inverso al usual para las separaciones con emulsiones dobles: el compuesto que se desea liberar va desde los glóbulos de la fase interna $\left(\mathrm{W}_{1}\right)$ a través de la membrana $(\mathrm{O})$ hacia la fase continua externa $\left(\mathrm{W}_{2}\right)$.

Se ha encontrado que la liberación del medicamento depende de la composición de la membrana, el tipo de droga a transferir, la concentración de la droga en la face interna y la composición de la fase interna y externa.

Uno de los problemas que se encuentran en la liberación controlada empleando emusliones dobles es el rompimiento de las membranas. Éste puede ocurrir debido a una baja estabilidad de la misma o porque las gotas se hinchan hasta llegar a romperse. El hinchamiento de las gotas se debe al paso de agua hacia la fase interna debido al gradiente de presión osmótica.

La mayoría de los estudios con emulsiones dobles se han realizado con emulsiones del tipo $\mathrm{W}_{1} / \mathrm{O} / \mathrm{W}_{2}$. Existen sin embargo estudios en los cuales la droga es liposoluble y se han utilizado emulsiones del tipo $\mathrm{O}_{1} / \mathrm{W} / \mathrm{O}_{2}$. Tal es el caso de la liberación controlada de la Pentazocina.

La inherente inestabilidad de las emulsiones que se asocia a la complejidad estructural de estos sistemas al igual que su uso para aplicaciones en alimentos es más limitado, esto por la falta de emulsificantes y estabilizadores con un grado alimenticio adecuado para las emulsiones interiores y exteriores. Existen varios factores que afectan la estabilidad de una emulsión $\mathrm{W}_{1} / \mathrm{O} / \mathrm{W}_{2}$. Entre los cuales tenemos: El método de preparación, naturaleza de los materiales entrampados, el volumen de las fases, la concentración y tipo de emulsionantes, 
los electrolitos presentes en el sistema y todos los ingredientes adicionados en la fase acuosa externa (Ôzer et al., 2000).

La eficacia y eficiencia en la aplicación de las emulsiones dobles está íntimamente ligada al uso de emulsionantes como surfactantes, biopolímeros, copolímeros, entre otros, que son utilizados para proveer estabilidad cinética y mantener su estructura por un largo periodo de tiempo (Pérez-Orozco et al., 2011).

\subsection{Solución de ecuaciones diferenciales por métodos numéricos}

Los métodos numéricos son una clase de métodos para resolver una amplia variedad de problemas matemáticos. Estos problemas se plantean a partir de la modelización matemática de fenómenos o procesos fisicoquímicos. Esta clase de métodos utilizan únicamente operaciones lógicas y aritméticas; por consiguiente, pueden implementarse sobre computadoras digitales.

Los métodos numéricos fueron desarrollados muchos años antes de la aparición de las computadoras electrónicas digitales. En efecto, un gran número de métodos numéricos usualmente utilizados datan de los comienzos de las matemáticas modernas. Sin embargo, el uso de tales métodos estuvo restringido hasta la aparición de las computadoras personales (PC's), incrementándose dramáticamente con la introducción de las computadoras electrónicas digitales.

La combinación de métodos numéricos y computadoras digitales constituye una poderosa herramienta para el análisis matemático. Por ejemplo, los métodos numéricos son capaces de manejar no linealidades, geometrías complejas y sistemas de ecuaciones acopladas que son necesarias para la simulación segura de muchos sistemas fisicoquímicos que se presentan en la ingeniería. Los enfoques analíticos rara vez son considerados, aún en problemas en donde podrían obtenerse soluciones analíticas, dado que los métodos numéricos son baratos, fáciles de emplear y con frecuencia se dispone de ellos en programas comerciales.

Los métodos numéricos son la parte de análisis numérico la cuál estudia la solución numérica a ecuaciones diferenciales ordinarias (EDO). Este campo también es conocido 
como Integración Numérica, pero alguna gente reserva este término para el cómputo de integrales.

Muchas ecuaciones diferenciales no se pueden solucionar analíticamente, en este caso se tienen que satisfacer con una aproximación a la solución, usando algoritmos que pueden ser utilizados en computadoras para encontrar tal aproximación. Un método alternativo es utilizar técnicas de cálculo para obtener una serie de la extensión de la solución.

Las ecuaciones diferenciales ocurren en muchas disciplinas científicas, por ejemplo en la mecánica, química, biología y economía. Además, algunos métodos de ecuaciones diferenciales parciales numéricas convierte la ecuación diferencial parcial en una ecuación diferencial ordinaria, que entonces puede ser solucionada.

En la búsqueda de una descripción cualitativa (Salvadori y Baron, 1992) de un determinado fenómeno físico, por lo general el ingeniero plantea un sistema de ecuaciones diferenciales ordinarias o parciales, válidas para determinado dominio, e impone sobre dicho sistema las condiciones apropiadas tanto de frontera como iniciales. En esta etapa, el modelo matemático está completo, y es aquí donde aparece la mayor dificultad, siendo que solo la forma más simple de ecuaciones, con fronteras geométricamente triviales es capaz de ser resuelta en forma exacta con los métodos matemáticos disponibles. Las ecuaciones diferenciales ordinarias con coeficientes constantes son uno de los pocos ejemplos para los cuales se dispone de procedimientos matemáticos clásicos de solución. Con el fin de evitar tales dificultades y lograr resolver el problema con la ayuda de computadoras, es necesario presentar el problema de una manera puramente algebraica. Mediante el proceso de discretización, el conjunto infinito de números que representan la función o funciones incógnitas en el dominio continuo es reemplazado por un número finito de parámetros incógnita, y este proceso requiere alguna forma de aproximación.

Los métodos numéricos para el estudio del comportamiento de sistemas dinámicos han cobrado fuerza en los últimos años por varias razones. Probablemente la más importante, es que puede accederse a computadoras altamente eficientes a un costo cada vez más bajo, lo que permite su uso para la resolución de problemas altamente complejos. Una segunda razón es que los métodos numéricos son en muchos casos la única alternativa posible para 
la resolución de los frecuentes problemas no-lineales muchas veces intratables analíticamente (González-Longatt). Por otra parte los problemas lineales continúan creciendo en magnitud, requiriendo un mayor esfuerzo para su solución.

\subsection{Método de Diferencias Finitas}

La teoría de Ecuaciones en Derivadas Parciales (EDP) se ha convertido en uno de los campos de estudio más importantes en matemáticas, debido a su frecuente aplicación en diferentes áreas de la física, ingeniería y otras ciencias. Entre las EDP más representativas se encuentran la Ecuación de Laplace, la Ecuación de onda y la Ecuación de calor.

Los métodos de aproximación analítica a la solución de EDP, proporcionan frecuentemente información útil acerca del comportamiento de la solución en valores críticos de la variable dependiente, pero tienden a ser más difíciles de aplicar que los métodos numéricos para solucionar ciertos tipos de ecuaciones diferenciales ordinarias y en derivadas parciales se encuentran:

1) Los datos de los problemas reales presentan siempre errores de medición, y el trabajo aritmético para la solución está limitado a un número finito de cifras significativas que resultan en errores de redondeo. Por lo tanto, incluso los métodos analíticos proporcionan resultados que son aproximaciones numéricas;

2) La evaluación numérica de las soluciones analíticas es a menudo una tarea laboriosa y computacionalmente ineficiente, mientras que los métodos numéricos generalmente proporcionan soluciones numéricas adecuadas, de manera más simple y eficiente (Smith, 1999).

De los métodos de aproximación numérica disponibles para resolver ecuaciones diferenciales, los más utilizados son el método de elementos finitos y el método de diferencias finitas. Este último es el método con el que se trabajó en este proyecto por lo que se describe a continuación.

El Método de Diferencias Finitas (Ramírez et al., 2005) es un método de carácter general que permite la resolución aproximada de ecuaciones diferenciales en derivadas parciales definidas en recintos finitos. Es de una gran sencillez conceptual y constituye un procedimiento muy adecuado para la resolución de una ecuación bidimensional. Es una 
clásica aproximación para encontrar la solución numérica de las ecuaciones que gobiernan el modelo matemático de un sistema continuo. El primer paso para la aplicación del método consiste en discretizar el recinto del plano en el que se quiere resolver la ecuación a través de un mallado, que por conveniencia es cuadrado. Los puntos de la malla están separados una distancia h en ambas direcciones x e y. Básicamente, en una solución por diferencias finitas, las derivadas son reemplazadas por aproximaciones en diferencias finitas, convirtiendo entonces un problema de ecuaciones diferenciales en un problema algebraico fácilmente resoluble por medios comunes (especialmente matriciales).

\subsection{Modelos de emulsión doble}

La resistencia eléctrica de las membranas de intercambio iónico dependen fuertemente de la concentración de la solución (Piotr et al., 2010). Aunque el número de transporte de iones en la membrana de intercambio iónico puede ser determinado experimentalmente a partir de mediciones de cronopotenciometría, los cálculos del modelo son una herramienta útil para predecir el número de transporte de iones.

Dichos cálculos se hacen sobre la base de la teoría del equilibrio de Donnan [Ecuación (5)], según la cual los cationes pasan a través de la membrana de intercambio catiónico como contra-iones, mientras que los aniones son rechazados (Sata, 2004; Strathmann, 2004) (sucede lo opuesto con membranas de intercambio aniónico.

$$
\ln \left[\left(\frac{a_{a}}{\bar{a}_{a}}\right)^{v_{a}} \cdot\left(\frac{a_{c}}{\bar{a}_{c}}\right)^{v_{c}}\right]=\frac{V_{a c}}{V_{w}} \ln \left(\frac{a_{w}}{\bar{a}_{w}}\right)
$$

Dónde: $a$ es la actividad en la solución y $\bar{a}$ es la actividad en la membrana, $v_{a}$ es el número de moles de aniones $v_{c}$ es el número de moles de cationes y $V$ es el volumen molar parcial de cualquiera de los electrolitos (subíndice ac) o agua (subíndice w). El subíndice a se refiere a anión, c para catión, ca al electrolito y w al agua.

Cuando $V_{a c} / V_{w}$ es igual al coeficiente de actividad $\gamma$, hay cambios en la ecuación de equilibrio de Donnan: 


$$
\frac{\bar{a}_{a}^{v_{a}} \cdot \bar{a}_{c}^{v_{c}}}{\bar{a}_{w}^{\gamma}}=\frac{a_{a}^{v_{a}} \cdot a_{c}^{\nu_{c}}}{a_{w}^{\gamma}}
$$

Cuando $\bar{a}_{w} \approx a_{w}$ y el coeficiente de actividad se supone que es igual a 1 (suposición razonable en el caso de soluciones salinas diluidas) la distribución de los iones $\mathrm{Na}^{+}$y $\mathrm{Cl}^{-}$ entre la membrana y la solución de una membrana de intercambio catiónico se equilibra con una solución acuosa de cloruro de sodio, se puede expresar como:

$$
C_{N a}^{m} \cdot C_{N a}^{m}=C_{N a} \cdot C_{C l}
$$

Dónde: $C_{N a}^{m}$ es la concentración de iones de sodio en la membrana (mol/l), $C_{C l}^{m}$ es la concentración de iones de cloruro en la membrana (mol/l), $C_{N a}$ es la concentración de iones de sodio en la solución (mol/l), $C_{C l}$ es la concentración de iones de cloruro en la solución $(\mathrm{mol} / \mathrm{l})$.

Dado que el Dr. Norman Li invento la membrana de emulsión líquida (ELM) en 1968 (Li, 1968) varios modelos matemáticos se han desarrollado (Bhowal y Datta, 1998, 2001; Chakraborty et al., 2003; El-Said et al., 2003; Kargari et al., 2006; Lee et al., 1998; Liu y Zhang, 1997; Reis y Carvalho, 2004; Yan et al., 1992) sin soluciones analíticas hasta que (Huifang et al., 2012) propusieron un modelo de transporte completo facilitado de tipo II para el proceso de la facilitación de ELM (Huang et al., 2009).

El modelo propuesto por Huifang et al. (2012) es un conjunto de ecuaciones simultaneas de las cuales se obtiene una solución analítica. Señalan que en un determinado intervalo de parámetros la ecuación de valor propio tiene una singularidad que da lugar a un término adicional de la solución analítica, cuyo impacto es más fuerte en la fase inicial de recuperación. Ching-Rong et al. (2009) presentan una solución de forma cerrada para un modelo matemático de una membrana de emulsión líquida, al igual que Huifang utilizan el mismo conjunto de ecuaciones para resolver dichas ecuaciones.

El modelo propuesto es un conjunto de ecuaciones simultáneas de donde se obtiene la solución analítica: 


$$
\begin{gathered}
V_{e} \frac{d C_{e}}{d t}=-N\left(4 \pi R^{2}\right) k\left(C_{e}-C_{e}^{*}\right), \\
-\left.N\left(4 \pi R^{2}\right) D_{e}\left(\frac{\partial C_{m}}{\partial r}\right)\right|_{r=R}=-N\left(4 \pi R^{2}\right) k\left(C_{e}-C_{e}^{*}\right)
\end{gathered}
$$

Condición inicial: $C_{e}=C_{e 0}$ cuando $t=0$

Donde $V_{e}$ es el volumen de la fase externa $\left(\mathrm{dm}^{3}\right), C_{e}$ es la concentración del soluto en la fase externa $\left(\mathrm{mol} / \mathrm{dm}^{3}\right), \mathrm{t}$ es el tiempo (s), $\mathrm{N}$ es el número total de glóbulos de la emulsión, $\mathrm{R}$ es el radio de los glóbulos, $k$ es el coeficiente de transferencia de masa externo $(\mathrm{m} / \mathrm{s})$, $C_{e}^{*}$ es la concentración del soluto en la interfaz de la membrana en la fase externa, $D_{e}$ difusividad efectiva del complejo soluto-portador en la fase de emulsión $\left(\mathrm{m}^{2} / \mathrm{s}\right) C_{m}$ concentración del complejo soluto-portador en la fase membrana $\left(\mathrm{mol} / \mathrm{dm}^{3}\right), \mathrm{r}$ es el radio interior en el glóbulo de la emulsión $(\mathrm{m}), k\left(C_{e}-C_{e}^{*}\right)$ es el flujo de transferencia de masa fuera de la interfaz de la membrana externa y $\left.D_{e}\left(\frac{\partial C_{m}}{\partial r}\right)\right|_{r=R}$ es el flujo de difusión de masa dentro de la interfaz de la membrana externa.

La ecuación de equilibrio es:

$$
C_{m}^{*}=m C_{e}
$$

Donde $C_{m}^{*}$ es la concentración del complejo soluto-portador en la interfaz de la fase exterior de la membrana (en la fase membrana), m es el coeficiente de distribución de extracción en la interfaz de la fase externa de la membrana.

$$
V_{m} \frac{\partial C_{m}}{\partial t}=\left(V_{m}+V_{i}\right) D_{e}\left[\frac{1}{r^{2}} \frac{\partial}{\partial r}\left(r^{2} \frac{\partial C_{m}}{\partial r}\right)\right]-V_{i} R_{x}
$$

Condiciones iniciales: $C_{m}=0$ para todo r, cuando t=0

Condiciones a la frontera: $C_{m}=$ finito, cuando $r=0 ; C_{m}=C_{m}^{*}$ cuando $r=R$ 
Donde $V_{m}$ es el volumen de la fase membrana $\left(\mathrm{dm}^{3}\right), V_{i}$ es el volumen de la fase interna $\left(\mathrm{dm}^{3}\right), R_{x}$ es la velocidad de desaparición del complejo soluto-portador en la fase membrana en la fase interna por extracción $\left(\mathrm{mol} / \mathrm{dm}^{3} / \mathrm{s}\right)$.

$$
R_{x}=\frac{\partial C_{i}(r, t)}{\partial t}
$$

Condición inicial: $C_{i}=0$ cuando t=0

Donde $C_{i}$ es la concentración del soluto en la fase interna.

$$
C_{i}=q C_{m}
$$

Donde q es el coeficiente de distribución de extracción en la interfase de la membrana en la fase interna.

Combinando las ecuaciones (11) y (13), se obtiene:

$$
\left(\frac{V_{m}+q V_{i}}{V_{m}+V_{i}}\right) \frac{\partial C_{m}}{\partial t}=D_{e}\left[\frac{1}{r^{2}} \frac{\partial}{\partial r}\left(r^{2} \frac{\partial C_{m}}{\partial r}\right)\right]
$$

Para un sistema de coordenadas esférico se puede usar la segunda ley de fick para describir el transporte de $\mathrm{NaCl}$ en las emulsiones dobles. El sistema se puede simplificar matemáticamente en un sistema de dos fases formado por el depósito de $\mathrm{NaCl}$ (en el interior de la emulsión $\left.\mathrm{W}_{1} / \mathrm{O}\right)$ y la fase continua $\left(\mathrm{W}_{2}\right)$. La transferencia de masa se explica utilizando difusividades dependientes de la concentración donde $\mathrm{C}_{\mathrm{t}}$ es la concentración de $\mathrm{NaCl}$ en la fase continua en el tiempo t, $\mathrm{r}$ es la coordenada radial discretizada a lo largo del radio del depósito (especificado mediante los datos del tamaño del glóbulo) y $\mathrm{D}_{\mathrm{e}}$ es el coeficiente de difusión dependiente de la concentración de $\mathrm{NaCl}$ de la emulsión interna.

Aplicaciones de liberación controlada en emulsiones dobles del tipo $\mathrm{W}_{1} / \mathrm{O} / \mathrm{W}_{2}$ que contienen cloruro de sodio $(\mathrm{NaCl})$ y gelatina en la fase acuosa interna, fueron desarrolladas por Lanny et al. (2012). 


$$
\frac{\partial C_{t}}{\partial t}=\frac{\partial}{\partial r}\left(D \frac{\partial C_{t}}{\partial r}\right)
$$

El modelo Fujita de volumen libre se puede utilizar para modelar la cinética de liberación de sal

$$
D=D_{e q} \exp \left(-\beta\left(1-\frac{C_{t}}{C_{e q}}\right)\right)
$$

Donde $\mathrm{D}_{\text {eq }}$ es el coeficiente de difusión de $\mathrm{NaCl}$ de la emulsión doble en el equilibrio, $\beta$ es un parámetro de escala adimensional que relaciona las difusividad inicial y de equilibrio y $\mathrm{C}_{\text {eq }}$ es la concentración de equilibrio de $\mathrm{NaCl}$ en la fase continua. Las ecuaciones pueden integrarse mediante un método de diferencias finitas. La ecuación anterior está sujeta a las siguientes condiciones iniciales y de frontera.

Condiciones iniciales:

$$
t=0 ; C_{t}=0
$$

Condiciones a la frontera:

$$
\begin{aligned}
& r=0 ; \frac{\partial C_{t}}{\partial r}=0 \\
& r=R_{e q} ; C_{t}=1
\end{aligned}
$$

Donde $\mathrm{R}_{\mathrm{eq}}$ es el tamaño del glóbulo en equilibrio.

Modelación de transporte de la emulsión en medios porosos es particularmente difícil porque las propiedades reológicas y físicas de las emulsiones son diferentes de los promedios de los componentes. Cortis y Ghezzehei (2007) mostraron una teoría que proporciona un paso importante hacia un modelo generalizado de flujo multifásico.

Los enfoques actuales de modelado se basan en las teorías de filtración, que no son apropiados para tratar adecuadamente las fluctuaciones de escala de poro de permeabilidad 
y la reducción de la permeabilidad absoluta que a menudo se encuentra durante el transporte de la emulsión.

El transporte de una emulsión en medios porosos se describe según Soo y C. J. (1986) como análogo al transporte de una fase coloidal.

El transporte coloidal es comúnmente modelado por la teoría de la filtración coloidal (CFT por sus siglas en inglés) como una extensión de la ecuación de advección-dispersión (ADE por sus siglas en inglés).

$$
\frac{\partial c}{\partial t}+\frac{\rho_{b}}{\theta} \frac{\partial s}{\partial t}=F[c(x, t)]
$$

Las longitudes efectuadas no adimensionales con respecto a la longitud de la columna L son:

$$
F[c(x, t)] \equiv-v\left[\lambda c+\frac{\partial c}{\partial x}-\alpha \frac{\partial^{2} c}{\partial x^{2}}\right]
$$

Es el operador de filtración clásica (con v la velocidad de poro y $\alpha$ la dispersividad) que actúa sobre la concentración del coloide c(x,t), y

$$
\frac{\partial s}{\partial t}=\frac{\theta}{\rho_{b}} k_{f} c-k_{r} s
$$

Donde $\theta$ es la porosidad, $\rho_{b}$ es la densidad del coloide, s es la concentración del coloide adsorbida reversiblemente sobre superficies sólidas y $k_{f}$ y $k_{r}$ son las tasas de adsorción directa e inversa, respectivamente. El parámetro $\lambda$ es el coeficiente de filtración, la fracción de la fase dispersa irreversiblemente absorbida, puede calcularse como:

$$
\lambda=-\ln (f)+\alpha \ln \left(f^{2}\right)
$$

Donde $f$ es la fracción eluída.

Para omitir las grandes complicaciones matemáticas que surgen cuando se intenta resolver un problema en 3D de la liberación en masa de las microesferas, se considera un modelo 
simplificado. De acuerdo a las hipótesis, en lugar de la fase dispersa ("nube" de microesferas) se admite el concepto de pseudo-fase homogénea y la introducción de una función adicional.

Un modelo de transferencia de masa que permite evaluar la influencia de los parámetros consecutivos en el proceso de liberación de fármacos a partir de emulsiones múltiples es propuesto por Wronski et al.(2012).

$C_{s}(t, r)$ que, junto con $C(t, r)$, satisfacen el siguiente problema de simetría esférica con valores en la frontera:

$$
\begin{gathered}
\frac{\partial C(t, r)}{\partial t}-D^{2} \Delta C(t, r)=k_{l} \cdot a \cdot\left[C_{s}(t, r)-C(t, r)\right] \cdot \frac{\phi}{1-\phi} \\
\frac{\partial C_{s}(t, r)}{\partial t}=k_{l} \cdot a \cdot\left[C(t, r)-C_{s}(t, r)\right] \\
\left.D^{2} \frac{\partial C(t, r)}{\partial r}\right|_{r=R}=-\left.h C(t, r)\right|_{r=R} \\
C(t, r)=C_{s}(t, r)=C_{s 0} \phi \text { para } t=t_{0}
\end{gathered}
$$

Donde $C_{s}=C_{s}^{*}$ es la concentración equivalente de la especie en microesferas respecto a la concentración de una especie en líquido en la gota de la emulsión $\left(\mathrm{mol} / \mathrm{m}^{3}\right), \varphi$ fracción de volumen de microesferas en la gota de la emulsión, $D_{2} \equiv D_{a}$ coeficiente de difusión del fármaco en la fase de la emulsión, $k_{l} \cdot a$ coeficiente volumétrico de transferencia de masa entre las microesferas y la fase continua de una gota $(\mathrm{m} / \mathrm{s})$, h coeficiente de transferencia de masa externo de las gotas al ambiente, $\Delta$ es un operador de Laplace en 3D escrito en coordenadas esféricas que en virtud de la simetría del problema se reduce a $\frac{1}{r^{2}} \frac{\partial}{\partial r} r^{2} \frac{\partial}{\partial r}$. El coeficiente de masa volumétrico $k_{l} \cdot a$ puede estimarse de acuerdo al modelo envolvente de Happel: 


$$
k_{l} a=\frac{3 D_{a}}{r_{i}^{2}} \frac{\phi^{1 / 3}}{\left(1-\phi^{1 / 3}\right)}
$$

En los parámetros de las ecuaciones: $h, k_{l} \cdot a, C_{s 0}, \varphi, D^{2}$ son constantes no negativas $(0<\varphi<1)$.

Modelado macroscópico de difusión y reacción química en sistemas de doble emulsión utilizando el método del promedio volumétrico es presentado por Morales-Zárate et al.(2008).

Las ecuaciones locales que regulan la transferencia de masa difusiva con procesos de reacción química son:

Fase $\sigma$ :

$$
\begin{aligned}
& \frac{\partial C_{A \sigma}}{\partial t}=\nabla \cdot\left(D_{A \sigma} \nabla C_{A \sigma}\right)-R_{\sigma} \\
& \frac{\partial C_{R \sigma}}{\partial t}=\nabla \cdot\left(D_{R \sigma} \nabla C_{R \sigma}\right)-R_{\sigma}
\end{aligned}
$$

Fase $\mu$ :

$$
\begin{aligned}
& \frac{\partial C_{A \mu}}{\partial t}=\nabla \cdot\left(D_{A \mu} \nabla C_{A \mu}\right)-R_{\mu} \\
& \frac{\partial C_{B \mu}}{\partial t}=\nabla \cdot\left(D_{B \mu} \nabla C_{B \mu}\right)+R_{\mu}
\end{aligned}
$$

Fase $\gamma$ :

$$
\frac{\partial C_{A \gamma}}{\partial t}=\nabla \cdot\left(D_{A \gamma} \nabla C_{A \gamma}\right)
$$

La cinética de las dos reacciones químicas que se producen en las fases $\sigma$ y $\mu$, se dan respectivamente por: 


$$
\begin{gathered}
R_{\sigma}=k_{\sigma} C_{A \sigma} C_{R \sigma} \\
R_{\mu}=k_{\mu}\left(C_{A \mu}-\frac{C_{B \mu}}{K_{\mu}}\right)
\end{gathered}
$$

La ecuación (31) considera la posibilidad de aumentar la capacidad de separación del sistema mediante la eliminación del soluto en la fase interna, la ecuación (32) incluye el mecanismo de transporte facilitado en la fase de la membrana. De ese modo, aunque las dos expresiones cinéticas se han adaptado para ser simples, la esencia del problema de separación se mantiene. Las ecuaciones de la (26)-(30) se asocian a las siguientes condiciones a la frontera:

En la interfase $\sigma \mu$

$$
\begin{gathered}
-\mathbf{n}_{\mu \sigma} \cdot D_{A \mu} \nabla C_{A \mu}=-\mathbf{n}_{\mu \sigma} \cdot D_{A \sigma} \nabla C_{A \sigma} \\
-\mathbf{n}_{\sigma \mu} \cdot D_{A \sigma} \nabla C_{A \sigma}=P_{\sigma \mu}\left(C_{A \sigma}-K_{e q}^{\mu \sigma} C_{A \mu}\right) \\
-\mathbf{n}_{\sigma \mu} \cdot D_{R \sigma} \nabla C_{R \sigma}=0 \\
-\mathbf{n}_{\mu \sigma} \cdot D_{B \mu} \nabla C_{B \mu}=0
\end{gathered}
$$

En la interfase $\mu \gamma$

$$
\begin{gathered}
-\mathbf{n}_{\mu \gamma} \cdot D_{A \mu} \nabla C_{A \mu}=-\mathbf{n}_{\mu \gamma} \cdot D_{A \gamma} \nabla C_{A \gamma} \\
-\mathbf{n}_{\mu \mu} \cdot D_{A \gamma} \nabla C_{A \gamma}=P_{\gamma \mu}\left(C_{A \gamma}-K_{e q}^{\mu \gamma} C_{A \mu}\right) \\
-\mathbf{n}_{\mu \gamma} \cdot D_{B \mu} \nabla C_{B \mu}=0
\end{gathered}
$$

Los coeficientes $P_{\sigma \mu}, P_{\mu \mu}, K_{e q}^{\mu \sigma}$ y $K_{e q}^{\mu \gamma}$ son funciones de las constantes de equilibrio locales, $n_{\mu \sigma}$ representa el vector normal unitario dirigido desde la fase $\mu$ hacia la fase $\sigma, K_{e q}^{\mu \sigma}$ y $K_{e q}^{\mu \gamma}$ son los coeficientes de distribución de equilibrio para el soluto A, mientras que la permeabilidad de la membrana de las interfaces $\sigma \mu$ у $\mathcal{\mu}$ son $P_{\sigma \mu}$ у $P_{\mu \mu}$ respectivamente. 


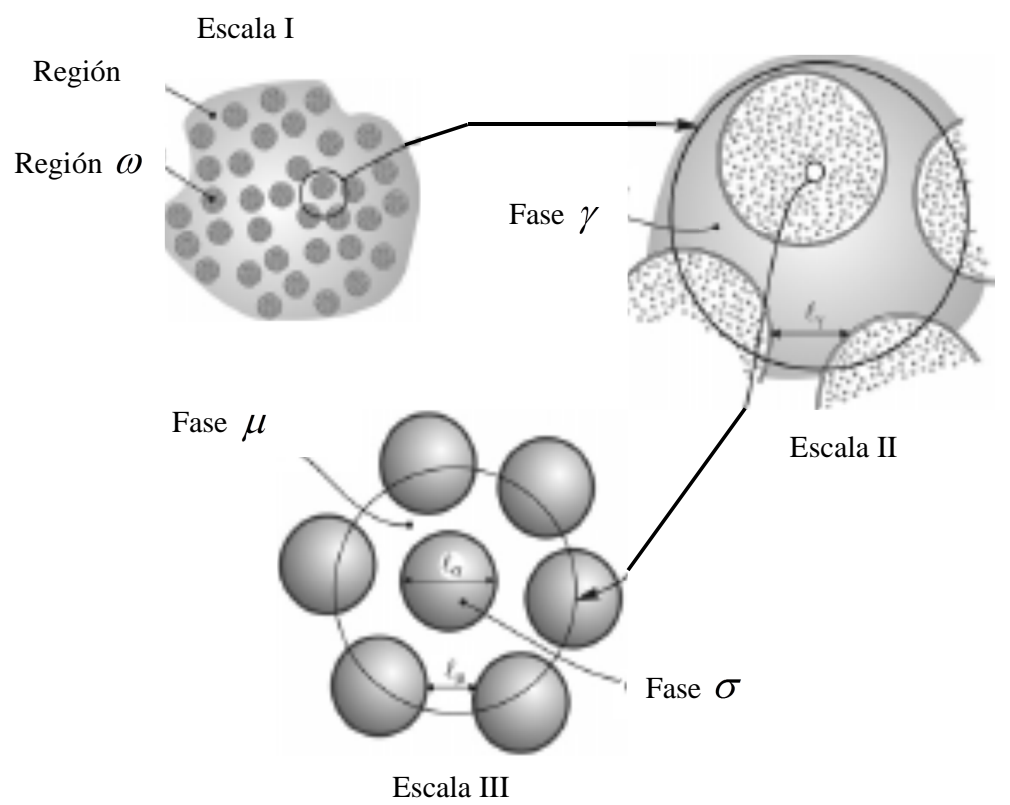

Figura 5. Sistema trifásico. Escala I: corresponde al sistema macroscópico; Escala II: representa una muestra de las gotas de membrana líquida rodeada de la fase externa $\gamma$, mientras que la escala III muestra las gotas en la fase de la membrana.

Un modelo de transferencia de masa general para la extracción de tensoactivo con membranas líquidas mediante el uso de la teoría de operador. El modelo, incorporando distintos parámetros, puede dar cuenta de los mecanismos de transferencia de masa para ambos transportes facilitados de tipo 1 y tipo 2, y los resultados son consistentes con las soluciones de perturbación de orden cero (Guoyu et al., 1997).

El transporte facilitado del tipo I, el soluto A de la fase de alimentación se difunde en la membrana para reaccionar con el reactivo interno $\mathrm{B}$ para producir el producto $\mathrm{P}_{1}$ :

$$
a A+b B \rightarrow P_{1}
$$

En transporte facilitado del tipo II soluto A de la fase de alimentación se difunde en la interfaz de los glóbulos de la emulsión donde reacciona con el portador C para formar complejos $\mathrm{P}_{2}$, y el complejo $\mathrm{P}_{2}$ se difunde en la interfaz de la fase interna donde reacciona con el reactivo interno B para liberar soluto A y portador C:

$$
a A+c C \rightarrow P_{2}
$$




$$
P_{2}+b B \rightarrow a A+c C
$$

Después de hacer algunas hipótesis, se obtienen las ecuaciones de difusión en los glóbulos de la emulsión, como sigue:

Glóbulos de la emulsión

$$
\begin{gathered}
\frac{D_{e}}{r^{2}} \frac{\partial}{\partial r}\left(r^{2} \frac{\partial C}{\partial r}\right)=\frac{\partial C}{\partial t}+\frac{\varphi_{i} K_{i} C}{a_{i}} \quad(0<r<R, t>0) \\
t=0, C=0 \quad(0 \leq r \leq R) \\
r=0, \frac{\partial C}{\partial r}=0 \quad(t \geq 0) \\
r=R, D_{e} \frac{\partial C}{\partial r}=K\left(C_{e}-\frac{C}{a_{e}}\right) \quad(t>0)
\end{gathered}
$$

Fase externa

$$
\begin{gathered}
-V_{e 0} \frac{\partial C_{e}}{\partial t}=\left.\frac{3}{R}\left(V_{i 0}+V_{m 0}\right)\left(l_{1} \frac{\partial C}{\partial r}+l_{2} C\right)\right|_{r=R} \\
t=0, C_{e}=C_{e 0}
\end{gathered}
$$

Donde C es la concentración de soluto dentro del glóbulo de la emulsión promediada sobre la membrana y la fase interna.

Para predecir el proceso de recubrimiento Luo et al. (2005) resentan un modelo numérico el cual mediante adecuados parámetros adimensionales, la ecuación gobernante, con las condiciones asociadas iniciales y de frontera son discretizadas utilizando la ecuación diferencial de Euler, y resuelven el sistema de ecuaciones utilizando MATLAB. 


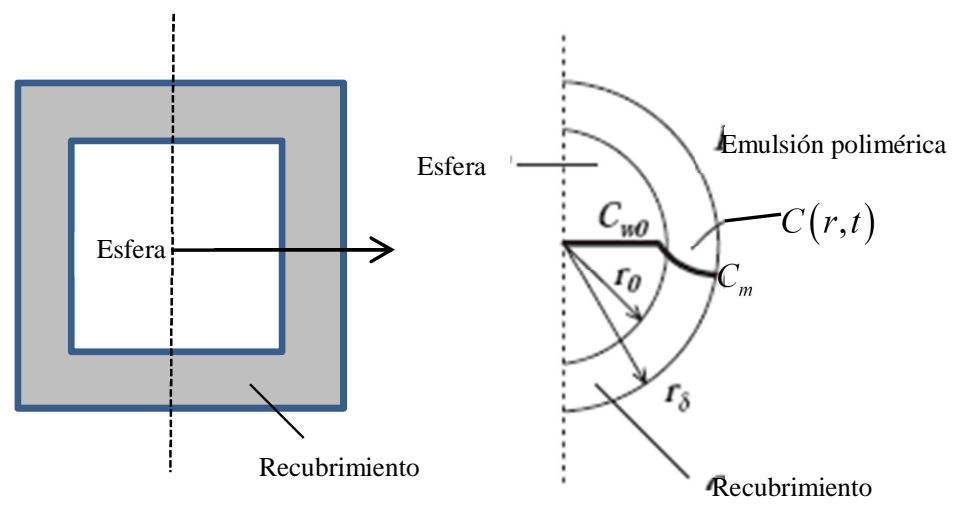

Figura 6. Esquema del proceso de recubrimiento de perlas de hidrogel

Consideran una gota esférica de hidrogel de radio $\mathrm{r}_{0}$, que se sumerge en una emulsión polimérica, la concentración inicial de agua en la gota es $C_{w 0}$. Las microgotas de polímero en contacto con la pared esférica se solidificaran y comenzaran a formar una capa porosa en la superficie exterior. Con el fin de implificar el proceso de modelado, se supone que la difusión del agua en la gota de hidrogel es muy rápida y su concentración se mantiene uniforme pero disminuye con el tiempo, es decir, la gota de hidrogel se puede tratar como un sistema agrupado de parámetros. Realizan algunas hipótesis y llegan a la ecuación gobernante que describe la concentración de agua en la capa de recubrimiento $C(r, t)$ es:

$$
\frac{\partial C}{\partial t}=D_{A B}\left[\frac{1}{r^{2}} \frac{\partial}{\partial r}\left(r^{2} \frac{\partial C}{\partial r}\right)\right]
$$

Condiciones iniciales y de frontera:

$$
\begin{gathered}
C\left(r_{0}, 0\right)=\frac{C_{w 0}}{K} \\
C\left(r_{0}, t\right)=\frac{C_{w}(t)}{K} \\
C\left(r_{\delta}(t), t\right)=C_{m} \\
-\left.D_{A B} \frac{\partial C}{\partial r}\right|_{r=r_{\delta}(t)}=\left(C_{m}-C_{\infty}\right) \frac{d r_{\delta}(t)}{d t}
\end{gathered}
$$




$$
\begin{gathered}
\frac{4}{3} \pi r_{0}^{3}\left[C_{w}(t)-C_{w 0}\right]=\int_{r_{0}}^{r_{\delta}(t)} 4 \pi r^{2}\left(C_{\infty}-C\right) d r \\
r_{\delta}(0)=r_{0}
\end{gathered}
$$

Donde $\mathrm{K}$ es el coeficiente de distribución de $\mathrm{H}_{2} \mathrm{O}$ en las gotas de hidrogel y la capa de revestimiento de polímero. Ecuación (53) es un balance de masa en la superficie de revestimiento y expresa el hecho de que el agua difundida en la superficie se equilibra con el agua absorbida por las microgotas poliméricas que se solidifican y se adhieren a la superficie exterior de las gotas. La ecuación (54) afirma que la pérdida de agua por la gota durante el intervalo de tiempo $(0, \mathrm{t})$ es igual al agua que se consume al aumentar la concentración de agua desde el valor inicial de $\mathrm{C}_{\infty}$ para su concentración final c(r,t) en la capa de recubrimiento. El objeto de este artículo es determinar simultáneamente el perfil de concentración de agua $\mathrm{C}(\mathrm{r}, \mathrm{t})$, la ubicación de las fronteras móviles y la concentración de agua en la gota de hidrogel $\mathrm{C}_{\mathrm{w}}(\mathrm{t})$.

Para proponer un modelo a partir del balance de masa Izquierdo-Gil et al. (2009) investigaron el flujo de sal a través de varias membranas comerciales de intercambio catiónico que separan dos soluciones de $\mathrm{NaCl}$ de alcohol-agua, con la misma concentración de alcohol, pero diferentes concentraciones de $\mathrm{NaCl}$.

El flujo de sal a través de la membrana (J) a partir de un balance de masa está dado por

$$
J=\frac{V}{A} \frac{d c_{1}}{d t}=-\frac{V}{A} \frac{d c_{2}}{d t}
$$

Donde $\mathrm{c}_{1} \mathrm{y}_{2} \mathrm{c}_{2}$ son las concentraciones de sal en las cámaras 1 y 2 (con $\mathrm{c}_{2}>\mathrm{c}_{1}$ ), $\mathrm{V}$ es el volumen de cada cámara y A es el área efectiva de la membrana.

El coeficiente de permeabilidad integral del sistema se define por

$$
P_{m s}=\frac{J}{c_{2}-c_{1}}
$$


Suponiendo que los volúmenes son constantes e iguales en ambas cámaras de las ecuaciones (56) y (57) se obtiene para $c_{1}(\mathrm{t})$ (concentración en la cámara diluida como función del tiempo):

$$
c_{1}(t)=\frac{c_{1}^{0}+c_{2}^{0}}{2}-a \frac{\Delta c^{0}}{2} \exp \left(-\frac{2 A P_{m s} t}{V}\right)
$$

Donde $c_{1}^{0}$ y $c_{2}^{0}$ son, respectivamente, la concentración inicial en la cámara diluida y concentrada, $\Delta c^{0}$ es la diferencia de concentración inicial. El parámetro a es una corrección de origen de tiempo, que considera que el tiempo de inicio del proceso de difusión y la hora inicial del cronómetro no eran exactamente las mismas. En general, el valor de a será muy cercano a uno. La ecuación (58) permite obtener $\mathrm{P}_{\mathrm{ms}}$ directamente del ajuste de los datos experimentales de $\mathrm{c}_{1}$ como una función del tiempo.

Suponiendo que la resistencia externa a la transferencia de masa no podría ser descuidada Simal et al. (2001) desarrollaron un modelo difusional y lo resuelven utilizando el método de diferencias finitas. El coeficiente de transferencia de masa se estimó de la literatura

El modelo propuesto fue desarrollado suponiendo dos componentes difusos durante el proceso. El agua, se mueve desde dentro del sólido hacia la superficie y de allí a la corriente del aire; mientras que el $\mathrm{NaCl}$, solo se mueve dentro del sólido. El estado transitorio de difusión de un componente, se expresa por la segunda ley de Fick.

$$
\begin{gathered}
\frac{\partial W}{\partial t}=D_{w}\left[\frac{\partial^{2} W}{\partial x^{2}}+\frac{\partial^{2} W}{\partial y^{2}}+\frac{\partial^{2} W}{\partial z^{2}}\right] \\
\frac{\partial S}{\partial t}=D_{S}\left[\frac{\partial^{2} S}{\partial x^{2}}+\frac{\partial^{2} S}{\partial y^{2}}+\frac{\partial^{2} S}{\partial z^{2}}\right]
\end{gathered}
$$

Donde W y S son la concentración local del agua y del $\mathrm{NaCl}$ respectivamente, y $\mathrm{D}_{\mathrm{w}}$ y $\mathrm{D}_{\mathrm{s}}$ son los coeficientes de difusión efectiva del agua y del $\mathrm{NaCl}$, que se consideran como constante. Se supone que no hay deformación de la partícula sólida durante el proceso. Como condición inicial consideran que el agua inicial y los perfiles de $\mathrm{NaCl}$ fueron los experimentales en el comienzo del proceso de maduración. 


$$
\begin{aligned}
& W(x, y, z, 0)=W_{\text {exp }}(x, y, z, 0) \\
& S(x, y, z, 0)=S_{\text {exp }}(x, y, z, 0)
\end{aligned}
$$

Las condiciones de frontera para el agua y la transferencia de $\mathrm{NaCl}$ se muestran a continuación

Tabla 1. Condiciones de frontera para el modelo de Simal et al. (2001)

\begin{tabular}{|l|l|}
\hline \multicolumn{1}{|c|}{ Agua } & \multicolumn{1}{|c|}{$\mathrm{NaCl}$} \\
\hline$\frac{\partial W(0, y, z, t)}{\partial x}=0$ & $\frac{\partial S(0, y, z, t)}{\partial x}=0$ \\
$\frac{\partial W(x, 0, z, t)}{\partial y}=0$ & $\frac{\partial S(x, 0, z, t)}{\partial y}=0$ \\
$\frac{\partial W(x, y, 0, t)}{\partial z}=0$ & $\frac{\partial S(x, y, 0, t)}{\partial z}=0$ \\
$-D_{w} \rho_{d m} \frac{\partial W(X, y, z, t)}{\partial x}=k_{c p x}\left(\varphi_{s x}-\varphi_{\infty}\right)$ & $-D_{S} \rho_{d m} \frac{\partial S(X, y, z, t)}{\partial x}=0$ \\
$-D_{w} \rho_{d m} \frac{\partial W(x, Y, z, t)}{\partial y}=k_{c p y}\left(\varphi_{s y}-\varphi_{\infty}\right)$ & $-D_{S} \rho_{d m} \frac{\partial S(x, Y, z, t)}{\partial y}=0$ \\
$-D_{w} \rho_{d m} \frac{\partial W(x, y, Z, t)}{\partial z}=k_{c p z}\left(\varphi_{s z}-\varphi_{\infty}\right)$ & $-D_{S} \rho_{d m} \frac{\partial S(x, y, Z, t)}{\partial z}=0$ \\
\hline
\end{tabular}

El coeficiente de masa para cada superficie fue estimado como sigue:

$$
\begin{aligned}
& k_{c p}=\frac{P_{S} M_{W}}{R T} k_{c} \\
& k_{c}=\frac{8}{\pi} D_{w a} \frac{1}{2 d}
\end{aligned}
$$




\section{Objetivos}

\section{Generales}

Solución y análisis numérico de un modelo de transporte de masa en un sistema de emulsión doble, y su aplicación para la predicción teórica de la liberación de soluto en sistemas $\mathrm{W}_{1} / \mathrm{O} / \mathrm{W}_{2}$.

\section{Objetivos particulares}

- Discretización del modelo por el método de diferencias finitas.

- Programación en Matlab.

- Análisis de estabilidad y consistencia numérica de la solución.

- Estimación de parámetros de transporte mediante el ajuste con datos experimentales reportados en la literatura.

- Predicción teórica en los perfiles de concentración en los sistemas de emulsión doble. 


\section{CAPÍTULO 2}

\section{Modelo matemático y discretización}

\subsection{Modelo de Morales-Zárate et al. (2008)}

Se propone un modelo matemático en coordenadas esféricas variables tanto en el tiempo como en el espacio y controlados por el mecanismo difusivo. Considerando un sistema de tres fases (ilustrado en la Figura 7) donde es posible identificar dos regiones homogéneas. La región $\omega$ está compuesta por una fase de membrana continua (fase $\mu$ ) y pequeñas gotitas dispersas (fase $\sigma$ ), mientras que la región $\eta$ corresponde a la fase continua externa $\gamma$ que rodea a la región $\omega$.

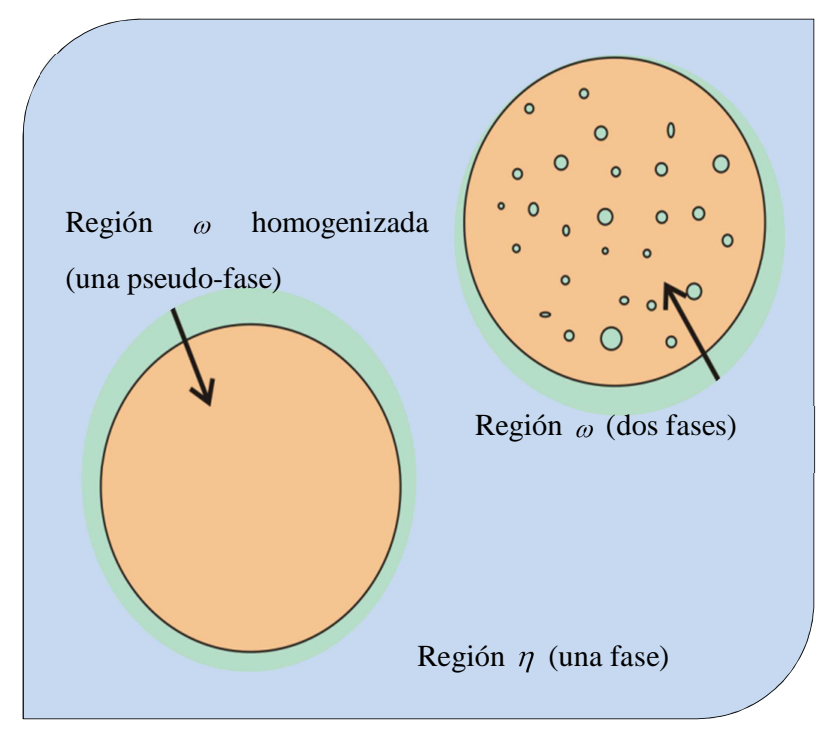

Figura 7. Esquema de un sistema de emulsión doble del tipo W/O/W.

Asumiendo equilibrio de masa local en la escala de Darcy la ecuación de transporte de masa en la región $\omega$ viene dada por (la región $\omega$ representa las gotas más grandes que contienen las gotas pequeñas dispersas en otras como se muestra en la Figura 7:

$$
\left(\varepsilon_{\mu \omega}+\varepsilon_{\sigma \omega} K_{e q}^{\mu \sigma}\right) \frac{\partial C_{\omega}}{\partial t}=\nabla \cdot\left[\mathbf{D}_{\omega} \nabla C_{\omega}\right]=\mathbf{D}_{\omega} \nabla^{2} C_{\omega}
$$


Donde $\varepsilon_{\mu \omega}$ y $\varepsilon_{\sigma \omega}$ son la fracción volumen de la fase membrana continua y las pequeñas gotas dispersas dentro de la región $\omega$, respectivamente; $K_{e q}^{\mu \sigma}$ es el coeficiente de distribución de equilibrio para el soluto entre la fase membrana y las pequeñas gotas, $C_{\omega}$ es la concentración del soluto, $\mathbf{D}_{\omega}$ es el tensor de difusividad efectiva en la región $\omega$ y $t$ es el tiempo.

Fuera de las gotas más grandes, la ecuación que rige los estados es:

$$
\frac{\partial C_{\eta}}{\partial t}=\nabla \cdot\left(D_{\eta} \nabla C_{\eta}\right) \frac{\partial C_{\eta}}{\partial t}=\nabla \cdot\left(D_{\eta} \nabla C_{\eta}\right)
$$

Donde $C_{\eta}$ es la concentración en la región $\eta$ y $D_{\eta}$ es la difusividad molecular en la región $\eta$

Las ecuaciones (65) y (66) se combinan con las condiciones de frontera en la superficie $\omega-\eta$ :

$$
\begin{gathered}
-\mathbf{n}_{\omega \eta} \cdot\left(D_{\eta} \nabla C_{\eta}\right)=-\mathbf{n}_{\omega \eta} \cdot\left(\mathbf{D}_{\omega} \nabla C_{\omega}\right) \\
-\mathbf{n}_{\omega \eta} \cdot\left(\mathbf{D}_{\omega} \cdot \nabla C_{\omega}\right)=P_{e f f}^{\eta}\left(C_{\eta}-K_{e f f}^{\eta \omega} C_{\omega}\right)
\end{gathered}
$$

Y se aplican las siguientes condiciones iniciales:

$$
C_{\omega}=C_{\omega}^{0} ; \quad C_{\eta}=C_{\eta}^{0}
$$

Aquí $\mathbf{n}_{\omega \eta}$ es el vector normal unitario que apunta desde la región $\omega$ a la región $\eta$ y $P_{e f f}^{\eta}$ y $K_{e f f}^{\eta \omega}$ son la permeabilidad efectiva y coeficiente de equilibrio respectivamente. Para una explicación más detallada sobre el modelo aquí expuesto hacemos referencia al trabajo de Morales-Zárate et al.(2008).

Generalmente, el ambiente que rodea a las gotas más grandes pueden asumirse como perfectamente mezclados, debido a algún proceso de agitación, lo que conduce a $C_{\eta}$ se considera independiente de la posición. Bajo estas condiciones podemos integrar la 
ecuación (66) en todo el dominio de $\eta$, y usando el teorema de la divergencia y las condiciones de frontera (68) y (69) se obtiene:

$$
\frac{\partial C_{\eta}}{\partial t}=a_{\eta \omega} P_{e f f}^{\eta}\left(\left.K_{e f f}^{\eta \omega} C_{\omega}\right|_{r=R_{\omega}}-C_{\eta}\right)
$$

Con $a_{\eta \omega}$ siendo el área interfacial entre la región $\eta$ y la región $\omega$ y $R_{\omega}$ es el radio en el dominio $\omega$. Nótese que con este procedimiento se ha obtenido una ecuación diferencial ordinaria en vez de la ecuación diferencial parcial original.

Operador laplaciano en coordenadas esféricas:

$$
\nabla^{2} C=\frac{1}{r^{2}} \frac{\partial}{\partial r}\left(r^{2} \frac{\partial C}{\partial r}\right)+\frac{1}{r^{2} \sin \theta} \frac{\partial}{\partial \theta}\left(\sin \theta \frac{\partial C}{\partial \theta}\right)+\frac{1}{r^{2} \sin ^{2} \theta} \frac{\partial^{2} C}{\partial \phi^{2}}
$$

En virtud de la simetría del problema se desprecian los gradientes en $\theta$ y $\phi$ por lo que (71) se reduce a:

$$
\nabla^{2} C=\frac{1}{r^{2}} \frac{\partial}{\partial r}\left(r^{2} \frac{\partial C}{\partial r}\right)
$$

Sustituyendo (72) en (65):

$$
\left(\varepsilon_{\mu \omega}+\varepsilon_{\sigma \omega} K_{e q}^{\mu \sigma}\right) \frac{\partial C_{\omega}}{\partial t}=D_{\omega}\left[\frac{1}{r^{2}} \frac{\partial}{\partial r}\left(r^{2} \frac{\partial C_{\omega}}{\partial r}\right)\right]
$$

Desarrollando el término entre corchetes de (73):

$$
\left[\frac{1}{r^{2}} \frac{\partial}{\partial r}\left(r^{2} \frac{\partial C_{\omega}}{\partial r}\right)\right]=\frac{1}{r^{2}}\left(r^{2} \frac{\partial^{2} C_{\omega}}{\partial r^{2}}+\frac{\partial C_{\omega}}{\partial r} 2 r\right)=\frac{\partial^{2} C_{\omega}}{\partial r^{2}}+\frac{2}{r} \frac{\partial C_{\omega}}{\partial r}
$$

Sustituimos (74) en (73) para obtener:

$$
\left(\varepsilon_{\mu \omega}+\varepsilon_{\sigma \omega} K_{e q}^{\mu \sigma}\right) \frac{\partial C_{\omega}}{\partial t}=D_{\omega}\left[\frac{\partial^{2} C_{\omega}}{\partial r^{2}}+\frac{2}{r} \frac{\partial C_{\omega}}{\partial r}\right]
$$


Con $r$ siendo la coordenada radial. Además hemos asumido la condición isotrópica del tensor $\mathbf{D}_{\omega}=D_{\omega} \mathbf{I}$.

\subsection{Discretización}

Discretizando la primera derivada obtenemos:

$$
\frac{\partial C_{i}}{\partial r}=\frac{C_{\eta_{i+1}}^{k}-C_{\eta_{i}}^{k}}{\Delta r}
$$

La discretización de la segunda derivada es:

$$
\frac{\partial^{2} C_{\omega}}{\partial r^{2}}=\frac{C_{\omega_{i+1}}^{k}-2 C_{\omega_{i}}^{k}+C_{\omega_{i-1}}^{k}}{(\Delta r)^{2}}
$$

Y la derivada temporal:

$$
\frac{\partial C_{\omega}}{\partial t}=\frac{C_{\omega_{i}}^{k+1}-C_{\omega_{i}}^{k}}{\Delta t}
$$

Dónde i indica la posición espacial y k indica el tiempo.

Sustituimos (76)-(78) en (75) para obtener la discretización en diferencias finitas de la ecuación diferencial en coordenadas esféricas:

$$
\begin{gathered}
\left(\varepsilon_{\mu \omega}+\varepsilon_{\sigma \omega} K_{e q}^{\mu \sigma}\right) \frac{C_{\omega_{i}}^{k+1}-C_{\omega_{i}}^{k}}{\Delta t}=D_{\omega}\left[\frac{C_{\omega_{i+1}}^{k}-2 C_{\omega_{i}}^{k}+C_{\omega_{i-1}}^{k}}{(\Delta r)^{2}}\right]+\frac{2 D_{\omega}}{r_{i}}\left[\frac{C_{\omega_{i+1}}^{k}-C_{\omega_{i}}^{k}}{\Delta r}\right] \\
\left(\varepsilon_{\mu \omega}+\varepsilon_{\sigma \omega} K_{e q}^{\mu \sigma}\right) C_{\omega_{i}}^{k+1}-C_{\omega_{i}}^{k}=\frac{D_{\omega} \Delta t}{(\Delta r)^{2}}\left[C_{\omega_{i+1}}^{k}-2 C_{\omega_{i}}^{k}+C_{\omega_{i-1}}^{k}\right]+\frac{2 D_{\omega} \Delta t}{r_{i} \Delta r}\left[C_{\omega_{i+1}}^{k}-C_{\omega_{i}}^{k}\right] \\
C_{\omega_{i}}^{k+1}=C_{\omega_{i}}^{k}+P\left[C_{\omega_{i+1}}^{k}-2 C_{\omega_{i}}^{k}+C_{\omega_{i-1}}^{k}\right]+\frac{Q}{r_{i}}\left[C_{\omega_{i+1}}^{k}-C_{\omega_{i}}^{k}\right]
\end{gathered}
$$

Dónde:

$$
P=\frac{D_{\omega} w}{\varepsilon_{\mu \omega}+\varepsilon_{\sigma \omega} K_{e q}^{\mu \sigma}} ;
$$




$$
\begin{aligned}
& w=\frac{\Delta t}{(\Delta r)^{2}} \mathrm{y} \\
& Q=\frac{2 * D_{\omega} * \Delta r * w}{\varepsilon_{\mu \omega}+\varepsilon_{\sigma \omega} K_{e q}^{\mu \sigma}}
\end{aligned}
$$

Las condiciones a la frontera en coordenadas esféricas se escriben como:

$$
D_{\omega} \frac{\partial C_{\omega}}{\partial r}=P_{e f f}^{\eta}\left(C_{\eta}-K_{e f f}^{\eta \omega} C_{\omega}\right)
$$

Discretizando ecuación (82):

$$
\begin{gathered}
D_{\omega}\left(\frac{C_{\omega_{n R}}^{k}-C_{\omega_{n R-1}}^{k}}{\Delta r}\right)=P_{e f f}^{\eta}\left(C_{\eta}^{k}-K_{e f f}^{\eta \omega} C_{\omega_{n R}}^{k}\right) \\
\left(\frac{C_{\omega_{n R}}^{k}-C_{\omega_{n R-1}}^{k}}{\Delta r}\right)=\frac{P_{e f f}^{\eta}}{D_{\omega}}\left(C_{\eta}^{k}-K_{e f f}^{\eta \omega} C_{\omega_{n R}}^{k}\right) \\
C_{\omega_{n R}}^{k}-C_{\omega_{n R-1}}^{k}=\frac{P_{e f f}^{\eta} \Delta r}{D_{\omega}}\left(C_{\eta}^{k}-K_{e f f}^{\eta \omega} C_{\omega_{n R}}^{k}\right) \\
C_{\omega_{n R}}^{k}-C_{\omega_{n R-1}}^{k}=S C_{\eta}^{k}-S K_{e f f}^{\eta \omega} C_{\omega_{n R}}^{k}
\end{gathered}
$$

La forma discretizada de la ecuación (82) queda como:

$$
C_{\omega_{n R}}^{k}=\frac{C_{\omega_{n R-1}}^{k}+S C_{\eta}^{k}}{\left(1+S K_{e f f}^{\eta \omega}\right)}
$$

Donde $S=\frac{P_{e f f}^{\eta} \Delta r}{D_{\omega}}$

Discretizando ecuación (66):

$$
\left(\frac{C_{\eta}^{k+1}-C_{\eta}^{k}}{\Delta t}\right)=-a_{\eta \omega} P_{e f f}^{\eta \omega}\left(C_{\eta}^{k}-K_{e f f}^{\eta \omega} C_{\omega_{n R}}^{k}\right)
$$




$$
\begin{gathered}
\frac{C_{\eta}^{k+1}-C_{\eta}^{k}}{\Delta t}=-a_{\eta \omega} P_{e f f}^{\eta \omega}\left(C_{\eta}^{k}-K_{e f f}^{\eta \omega} C_{\omega_{n R}}^{k}\right) \\
C_{\eta}^{k+1}-C_{\eta}^{k}=-a_{\eta \omega} P_{e f f}^{\eta \omega} \Delta t\left(C_{\eta}^{k}-K_{e f f}^{\eta \omega} C_{\omega_{n R}}^{k}\right)
\end{gathered}
$$

La ecuación diferencial de forma discretizada en diferencias finitas es:

$$
C_{\eta}^{k+1}=C_{\eta}^{k}-a_{\eta \omega} P_{e f f}^{\eta \omega} \Delta t\left(C_{\eta}^{k}-K_{e f f}^{\eta \omega} C_{\omega_{n R}}^{k}\right)
$$

En el anexo B se muestra el modelado de la transferencia de masa en la microescala. 


\section{CAPÍTULO 3}

\section{Análisis de la estabilidad y consistencia de la solución numérica}

\subsection{Comportamiento numérico}

En primer lugar, se explora la consistencia de los resultados numéricos en relación con el tamaño del paso del tiempo. La fijación del número de nodos para la dirección $r$ son 100, los valores de $B i_{m}$ y $\psi=1$, y la concentración inicial $c_{\eta}^{0}=0$, en la Figura 8 se muestran la evolución en la concentración en la región $\eta$ para varios valores de la relación de $\gamma$. Nótese que, prácticamente, para $\gamma=0.2973$ hemos llegado a una solución de malla independiente. Observe que, incluso para una malla relativamente gruesa (en comparación con aquellos que se utilizan en métodos simples de diferencia explícita) se evitan inestabilidades numéricas debido a la propagación del error $\mathbf{O}(\Delta \hat{r})^{2}$ y $\mathbf{O}(\Delta \tau)^{2}$.

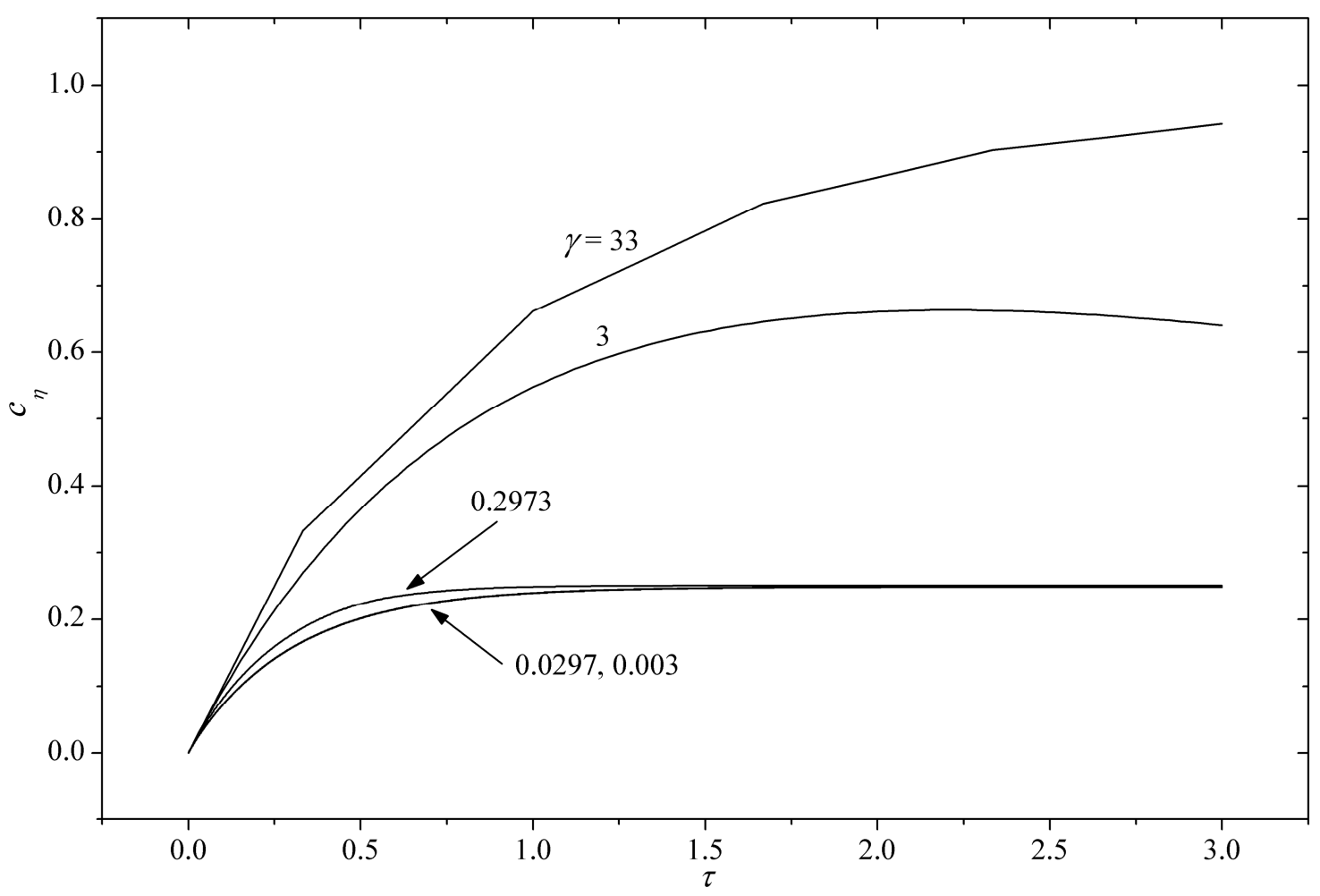

Figura 8. Evolución de la concentración en la región $\eta$ para diferentes valores de $\gamma$. Aquí se tomó

$$
N_{r}=100, c_{\eta}^{0}=0 \mathbf{y} B i_{m}=\psi=1 \text {. }
$$


Dónde:

$$
c_{\eta}=\frac{C_{\eta}-K_{e f f}^{\eta \omega} C_{\eta}^{0}}{K_{e f f}^{\eta \omega}\left(C_{\omega}^{0}-C_{\eta}^{0}\right)} ; B i_{m}=\frac{P_{e f f}^{\eta} R_{\omega} K_{e f f}^{\eta \omega}}{D_{\omega}} ; \psi=\frac{a_{\eta \omega} D_{\omega}^{2}}{R_{\omega}^{3} K_{e f f}^{\eta \omega}\left(\varepsilon_{\mu \omega}+\varepsilon_{\sigma \omega} K_{e q}^{\mu \sigma}\right)}
$$

Ahora, los perfiles de concentración dentro del dominio $\omega$ se muestran en la Figura 9 como función del tiempo. Prácticamente para $\tau=3$ el estado estacionario se alcanza. Siempre y cuando se utilice una malla de rejilla fina, la solución finalmente colapsará en una curva única. Se encontró que por causa de cálculos consistentes con una malla $N_{r}>50$ se puede utilizar.

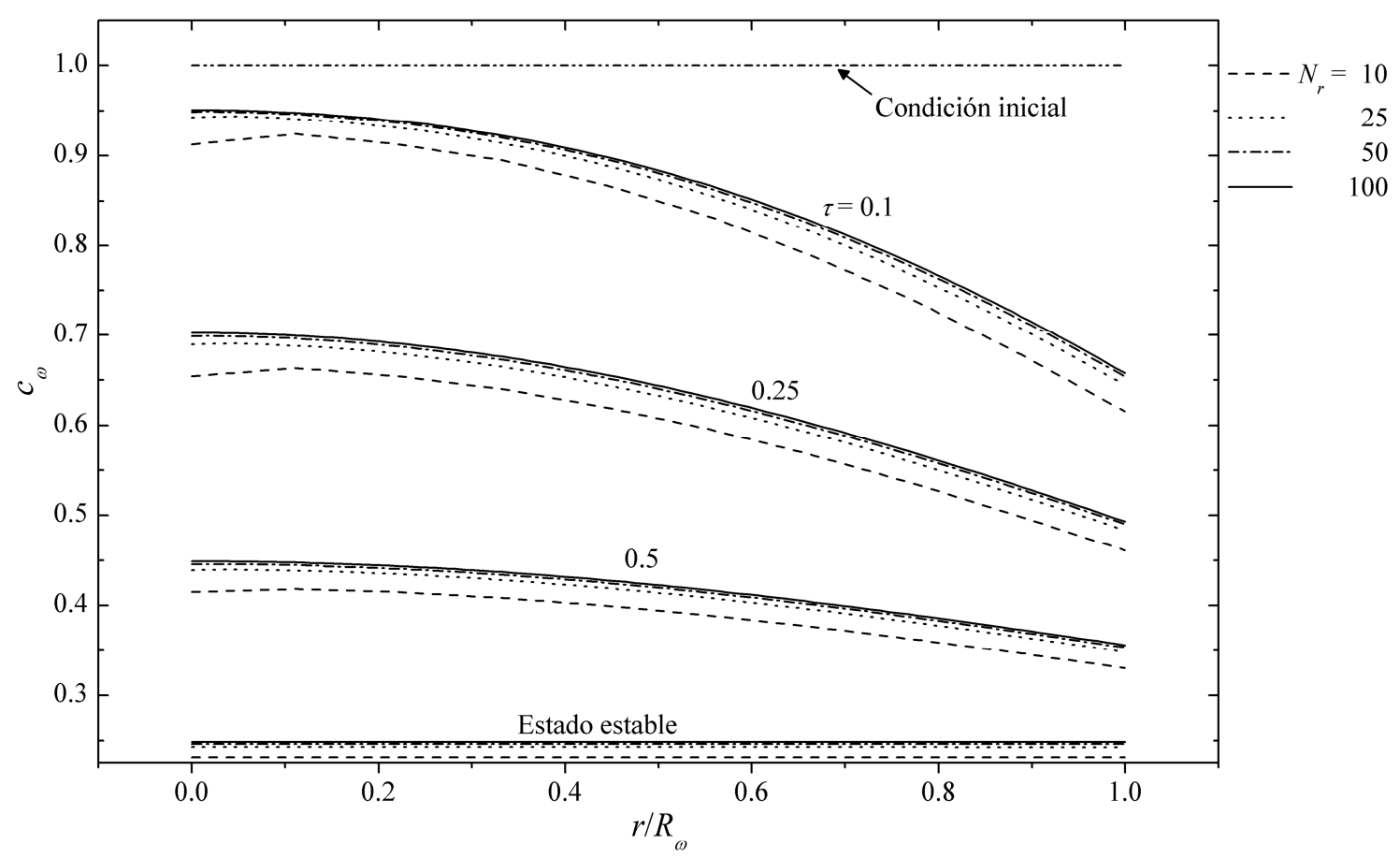

Figura 9. Perfiles de concentración en el dominio $\omega$ como función del radio adimensional y tiempo. Se

fijó el número de nodos en el tiempo para ser $N_{r} 100, c_{\eta}^{0}=0$ y $B i_{m}=\psi=1$.

Con el fin de resumir una región con los valores prescritos del par $B i_{m}-\psi$ con malla independiente de los resultados, una relación mínima de tamaños de paso se define como el valor de $\gamma$ que satisfacen un error menor que $0.01 \%$, en este caso el error es definido como: 


$$
\text { error }=\operatorname{abs}\left(\frac{\left.c_{\eta}\right|_{\tau=T} ^{\gamma-\varepsilon}-\left.c_{\eta}\right|_{\tau=T} ^{\gamma}}{\left.c_{\eta}\right|_{\tau=T} ^{\gamma-\varepsilon}}\right)
$$

Para aclarar la nomenclatura usada aquí, se ejemplifican con el término $\left.c_{\eta}\right|_{\tau=T} ^{\varphi}$ lo que indica que la concentración $c_{\eta}$ es que una resultante de una simulación con una relación de tamaños de la etapa $\varphi$ y evaluados en el tiempo T. en este respecto, el parámetro T se toma como el tiempo total no adimensional de la simulación, y el decremento $\varepsilon$ era tal que la relación $\mathrm{N}_{\mathrm{t}} / \mathrm{N}_{\mathrm{r}}$ siguió la secuencia:

$$
\frac{N_{t}}{N_{r}}=1,101,201,301,401, \ldots
$$

$\mathrm{N}_{\mathrm{t}}$ representa el número de nodos para la discretización de $\tau$.

De esta manera, una zona con malla independiente de los resultados se presenta en la Figura 10. Tenga en cuenta que el número de Biot de masa es el parámetro principal que afecta el valor mínimo de $\gamma\left(\gamma_{\text {min }}\right)$ y esto tiene una dependencia débil con $\psi$. Es obvio que una zona más restringida de malla independiente puede encontrarse mediante la imposición del error definido en la ecuación (93) cumple un valor menor de $0.01 \%$. 


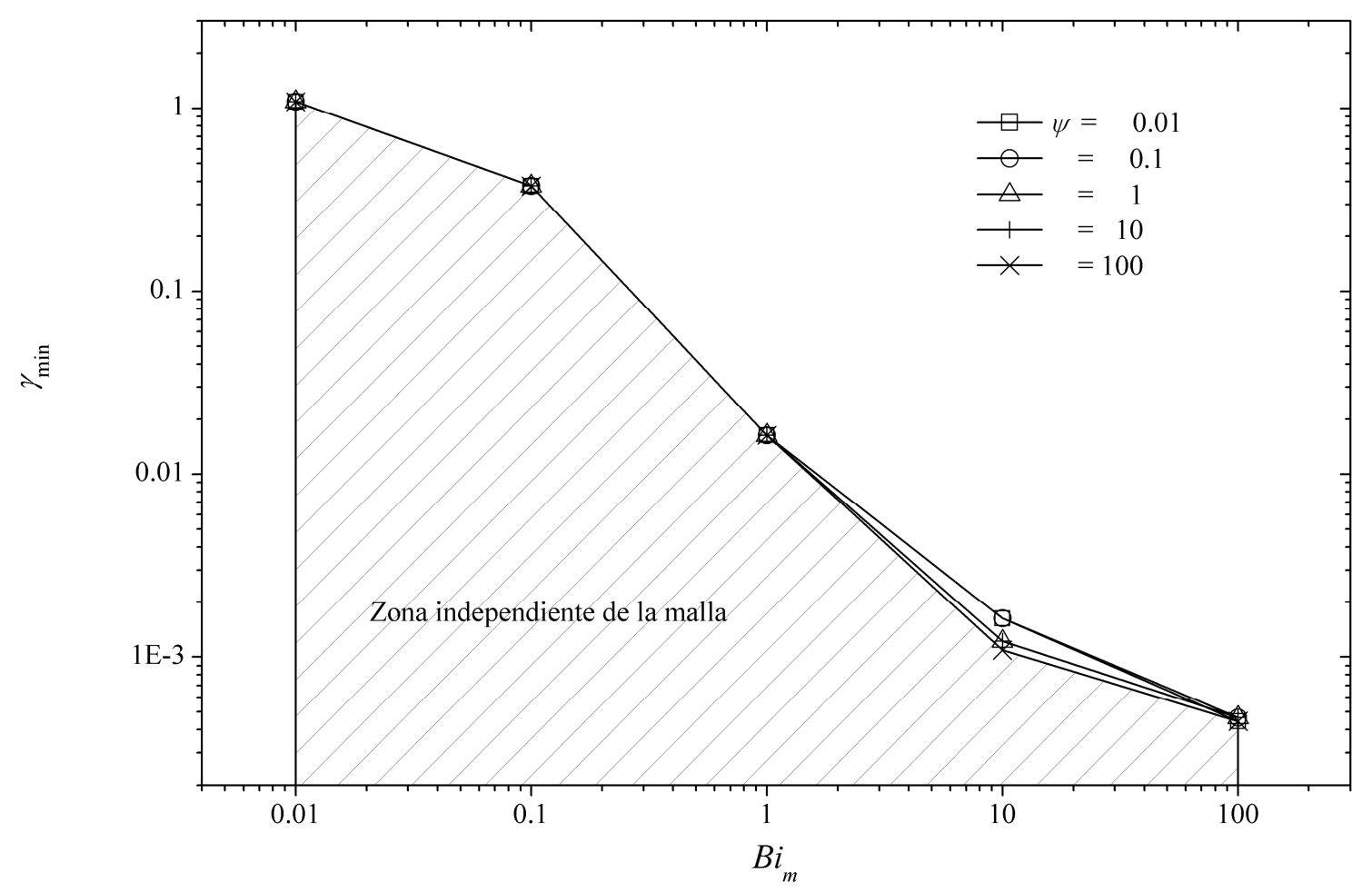

Figura 10. Efecto del número de Biot de masa y el parámetro $\psi$ en la relación mínima de tamaños de paso $\gamma$. Aquí se tomó $\mathbf{N}_{\mathbf{r}}=\mathbf{5 0}$ y $c_{\eta}^{0}=0$.

\subsection{Estudio paramétrico}

En el modelo matemático hay varios coeficientes de equilibrio y transporte eficaces. Sin embargo, algunos de ellos han predefinido los valores como el coeficiente de $K_{\text {eff }}^{\omega \eta}$ que en condiciones de equilibrio termodinámico toma el valor 1 (Morales-Zárate et al., 2008). Además, la fracción de volumen dentro del dominio $\omega \varepsilon_{\mu \omega}$ está limitada por la fracción de volumen restante $\varepsilon_{\sigma \omega}\left(\varepsilon_{\mu \omega}=1-\varepsilon_{\sigma \omega}\right.$ para las dos fases del sistema), o el caso contrario es cierto. Por lo tanto tenemos 3 parámetros efectivos que influyen en el transporte de masa en el sistema de emulsión doble: la difusividad efectiva $D_{\omega}$, cuantificar la velocidad de difusión del soluto en el dominio $\omega$, el producto $a_{\eta \omega} P_{e f f}^{\eta}$ cuantifica la transferencia de masa entre los dominios homogéneos, y el producto de equilibrio $\varepsilon_{\sigma \omega} K_{e q}^{\mu \sigma}$ que dicta el tiempo necesario para alcanzar un estado independiente a tiempo parcial. Sin embargo, tenga en 
cuenta que dichos parámetros están involucrados en el tiempo adimensional $\tau$, el número de Biot de masa $B i_{m}$ y el parámetro $\psi$.

Debido a que la variable independiente t está involucrado en $\tau$, no es posible manipular el estudio paramétrico. Así, solamente tenemos los grados de libertad de $B i_{m}$ у $\psi$.

En la Figura 11 se muestran la concentración no adimensional en el fluido externo, como función del número de Biot de masa y el parámetro $\psi$

Como se indica en la ecuación (92) de una manera general el número de Biot cuantifica la relación de transferencia de masa desde el dominio $\omega$ hasta $\eta$ con el transporte de masa por difusión en el mecanismo del dominio $\omega$. Para una tasa fija del transporte de masa difusivo, se puede pensar en el $\mathrm{Bi}_{\mathrm{m}}$ como la facilidad que el soluto tiene que moverse a través del límite $\omega-\eta$. Aquí hay que destacar que el soluto puede ser transferido desde el dominio $\omega$ al $\eta$, o también en sentido inverso dependiendo de las condiciones iniciales en el sistema. Las ideas aquí expuestas pueden ser observadas en el comportamiento de la concentración no dimensional que se muestra en la Figura 11a). Mientras el número de Biot de masa aumenta, la pendiente inicial de las curvas se hace más grande, lo que significa que una liberación más rápida del soluto está ocurriendo.

En la Figura 11b) el efecto de los parámetros $\psi$ en $c_{\eta}$ es trazado. Como se indica en la ecuación (92), el parámetro $\psi$ es la relación entre el transporte difusivo de masa del soluto y la constante de equilibrio. Así los valores más altos del parámetro $\psi$ indican la liberación rápida de soluto al líquido externo. Es un efecto similar al inducido por el número de Biot de masa, sin embargo, la concentración final estacionaria aumenta y el tiempo necesario para alcanzar un estado estacionario es menor. Claramente, este efecto es debido a la constante de equilibrio contenido en la definición de $\psi$. 
a)

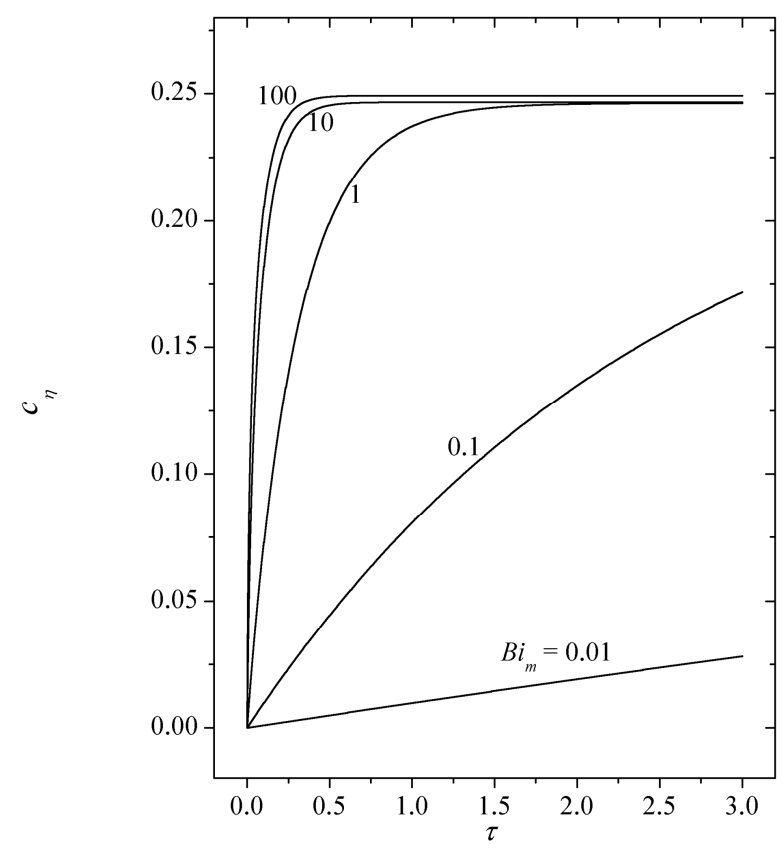

b)

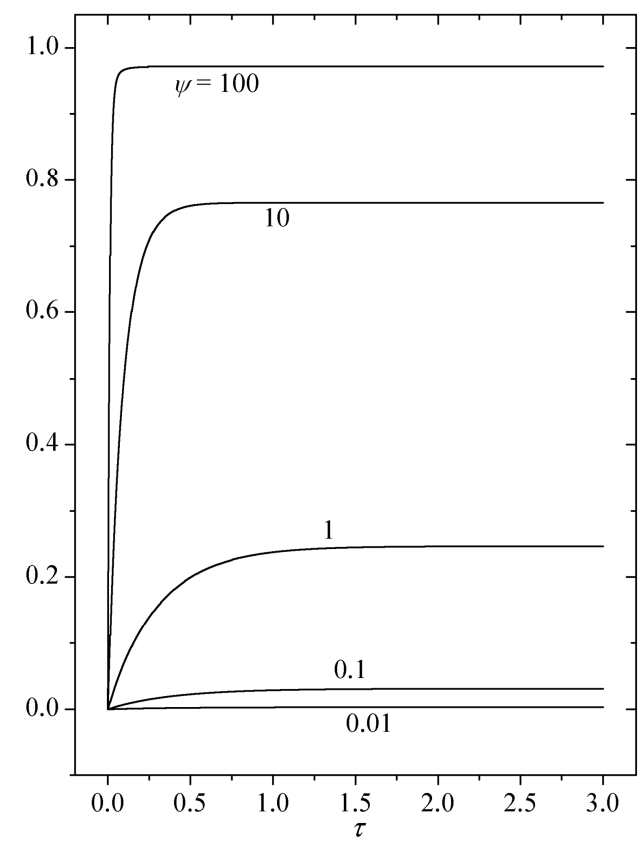

Figura 11. Efecto de a) el número de Biot de masa y b) el parámetro $\psi$ en la concentración de soluto

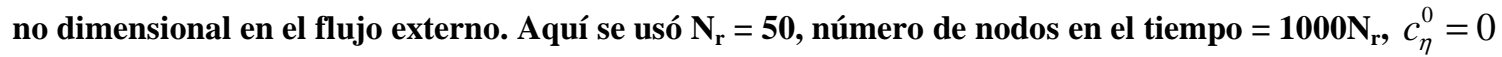
y siempre que sea aplicable $B \mathbf{i}_{\mathrm{m}}$ o $\psi=1$.

Los perfiles de concentración del soluto en el interior de las gotas grandes como funciones del número de Biot de masa y el parámetro $\psi$ se muestran en la Figura 12. Podemos observar que cuasi perfiles lineales se obtienen para la mayoría de los casos, lo que significa que los gradientes de concentración dentro del dominio $\omega$ es, generalmente, pequeña. En este caso los parámetros $\mathrm{Bi}_{\mathrm{m}} \mathrm{y} \psi$ tienen efectos opuestos sobre los perfiles de concentración. Como los resultados corresponden al momento $\tau=1$, algunos casos prácticamente alcanzan el estado estacionario (para $\mathrm{Bi}_{\mathrm{m}}, \psi=10,100$ ) que coincide con los resultados presentados en la Figura 11. Sin embargo, como se mencionó anteriormente, hay que destacar que el número de Biot de masa no modifica la concentración de estado estacionario, mientas que el parámetro $\psi$ lo hace. 
a)

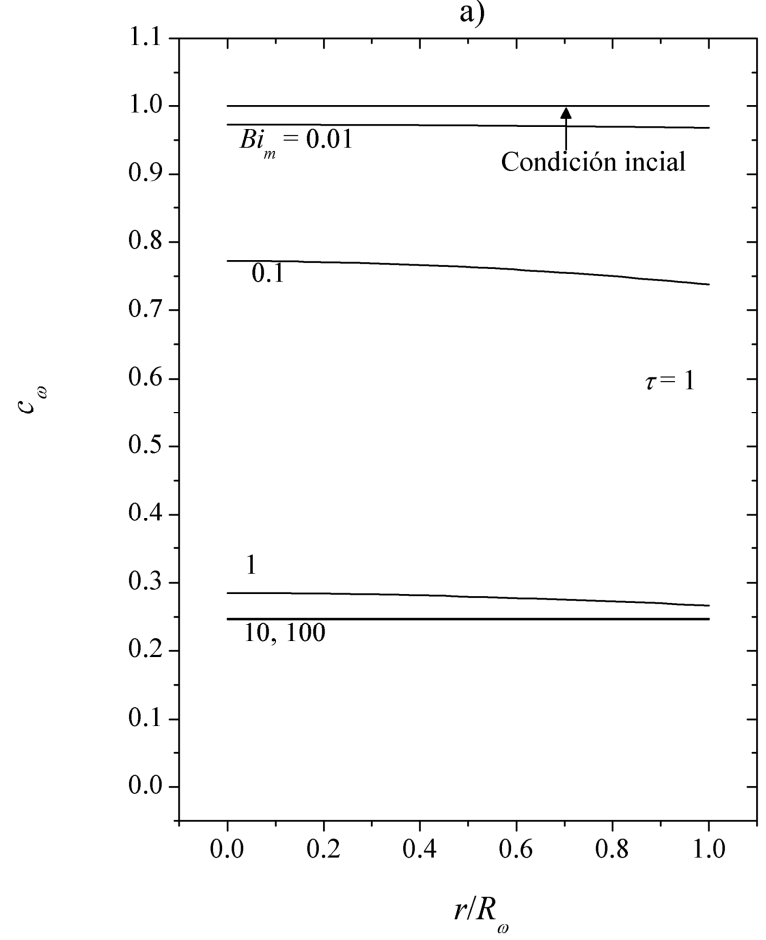

b)

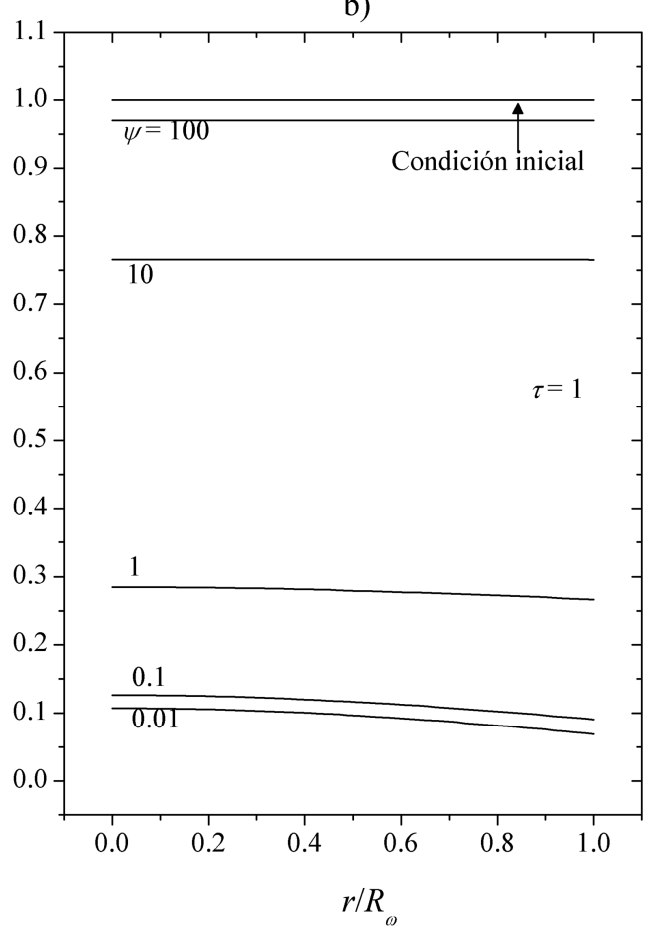

Figura 12. Efecto de a) el número de Biot de masa y b) el parámetro $\psi$ en la concentración de soluto

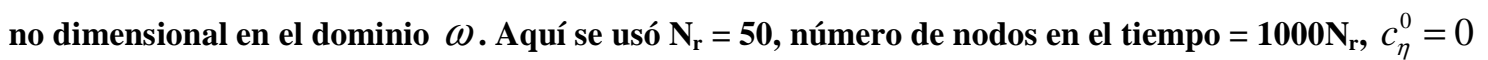
y siempre que sea aplicable $\mathrm{Bi}_{\mathrm{m}} \mathrm{o} \psi=1$.

Complementariamente, se puede concluir que:

$$
\begin{array}{r}
\underbrace{\frac{\partial c_{\eta}}{\partial \tau} \rightarrow \infty,\left.\frac{\partial c_{\omega}}{\partial \hat{r}}\right|_{\hat{r}=1} \rightarrow \infty}_{\text {en los primeros tiempos }} \text { y } \underbrace{\text { cuando } \quad B i_{m} \rightarrow \infty}_{\text {en tiempos posteriores }} \begin{array}{c}
\left.c_{\eta} \rightarrow c_{\omega}\right|_{\hat{r}=1}, \frac{\partial c_{\eta}}{\partial \tau} \rightarrow 0,\left.\frac{\partial c_{\omega}}{\partial \hat{r}}\right|_{\hat{r}=1} \\
\underbrace{\frac{\partial c_{\eta}}{\partial \tau} \rightarrow 0,\left.\frac{\partial c_{\omega}}{\partial \hat{r}}\right|_{\hat{r}=1} \rightarrow 0}_{\text {para todo el tiempo }}
\end{array} \\
\text { cuando } B i_{m} \rightarrow 0
\end{array}
$$

Y

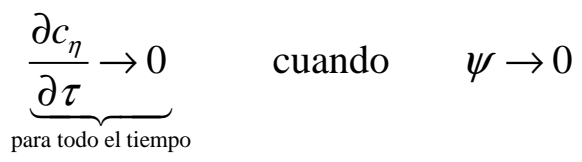


Estos resultados pueden ser corroborados con los resultados mostrados en las Figuras $11 \mathrm{y}$ 12.

En la Figura 13 se presenta el tiempo requerido para alcanzar la solución del estado estacionario $\left(\tau_{s s}\right)$ como función del número de Biot de masa y el parámetro $\psi$. Como uno de los criterios a considerar en el sistema de estado estacionario, hacemos cumplir que la concentración en la región $\eta$ satisface

$$
100 \mathrm{abs}\left(\frac{c_{\eta}^{k+1}-c_{\eta}^{k}}{c_{\eta}^{k+1}}\right) \leq 1 \times 10^{-6}
$$

Aquí $\mathrm{k}$ es el nivel de tiempo discretizada.

Se encontró que para pequeños valores de los parámetros de transporte, aumenta el tiempo necesario para alcanzar notablemente el estado estacionario. En general, con el fin de tener una rápida liberación del soluto se recomienda desarrollar condiciones con altos valores del número de Biot de masa y/o $\psi$. Además, para aplicaciones técnicas donde la condición requerida del soluto sea gradual y lenta, entonces los valores pequeños del $\mathrm{Bi}_{\mathrm{m}} \mathrm{y} \psi$ son los adecuados. Con estas ideas en mente, y mediante el uso de las definiciones en la ecuación (92), se sugieren algunas reglas heurísticas para dos casos de funcionamiento en la Tabla 2. Sostenemos que algunos factores pueden lograrse fácilmente mediante la manipulación de la configuración geométrica de las fases. Por ejemplo, al aumentar el tiempo de agitación durante la preparación de las emulsiones, el tamaño de las gotas se puede reducir y en consecuencia también la zona interfacial es modificada (factores de 2 y 3). Además, durante el proceso de liberación de soluto la agitación puede aumentar el coeficiente de transferencia de masa de intercambio $P_{e f f}^{\eta}$.

Además algunos factores como el 1, 4 y 5 sólo se pueden modificar mediante la variación de la temperatura, presión, tipo de disolventes y/o tensoactivos utilizados, e incluso la concentración de especies químicas en el sistema (agente tensoactivo, soluto, etc.). Estos factores pueden ser más difíciles de entender y llevar a cabo, y en este caso, la experiencia de los investigadores del laboratorio desempeña un papel crucial en el diseño de membranas para una aplicación específica. 


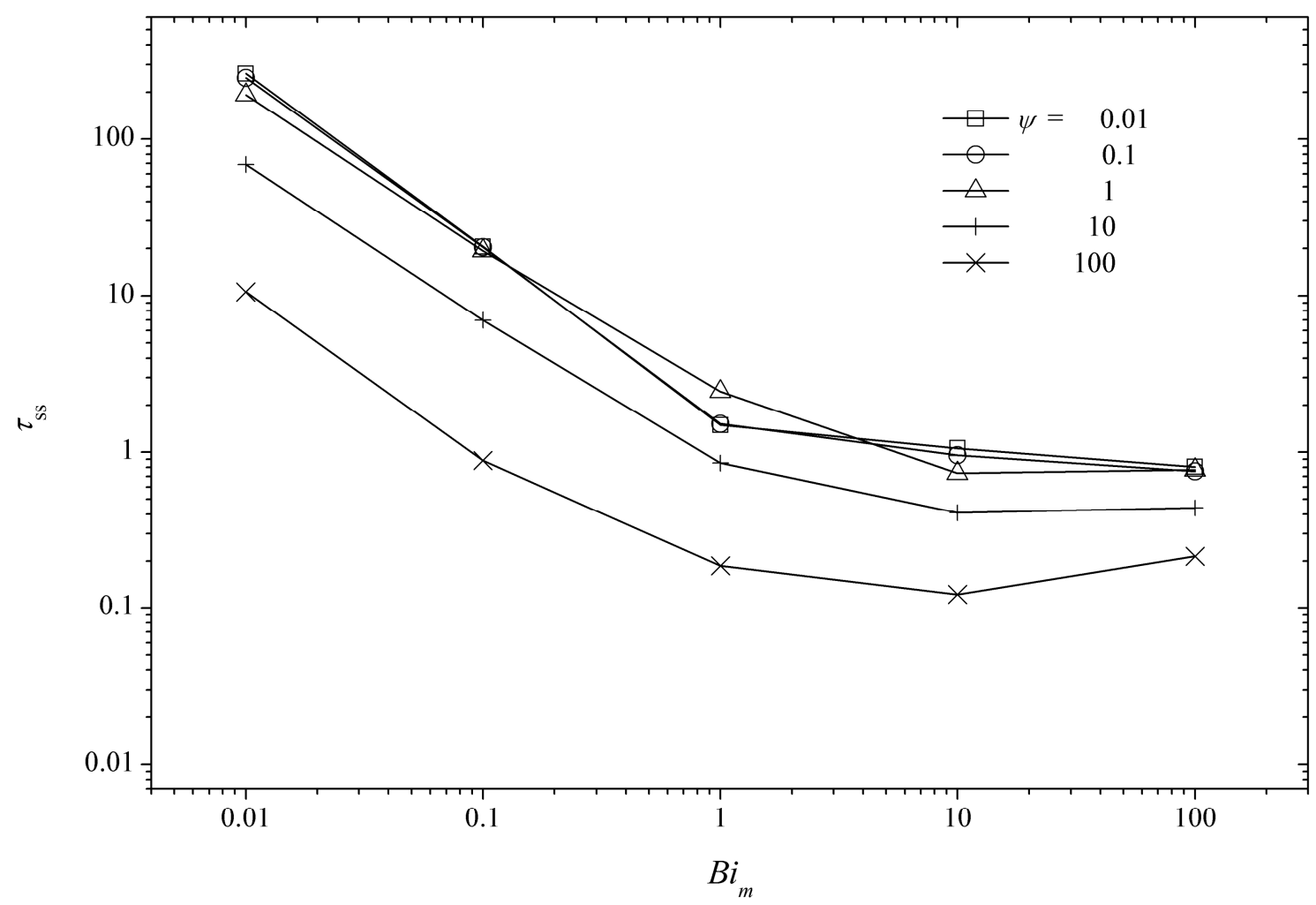

Figura 13. Tiempo necesario para llegar a la solución de estado estacionario en función de número de Biot de masa y el parámetro $\psi$. Aquí se tomó $c_{\eta}^{0}=0, N_{r}=50$ y $\gamma=5.88 \times 10^{-4}$.

Tabla 2. Algunas reglas heurísticas para aumentar o disminuir la liberación de soluto.

\begin{tabular}{lll}
\hline \hline Factor & \multicolumn{1}{c}{ Rápida liberación del soluto } & \multicolumn{1}{c}{ Liberación más lenta del soluto } \\
\hline \hline 1 & -Aumentar el intercambio del & -Disminuir el intercambio del \\
& coeficiente de transferencia de masa & coeficiente de transferencia de masa \\
& $P_{e f f}^{\eta}$ & $P_{e f f}^{\eta}$ \\
2 & - Reducir el tamaño de las gotas $R_{\omega}$ & - -Aumentar el tamaño de las gotas $R_{\omega}$ \\
3 & -Aumentar el área interfacial $a_{\eta \omega}$ & -Disminuir el área interfacial $a_{\eta \omega}$ \\
4 & - Aumentar la difusión efectiva dentro & -Disminuir la difusión efectiva dentro \\
& de las gotas $D_{\omega}$ & de las gotas $D_{\omega}$ \\
5 & - Disminuir la constante de equilibrio & -Incrementar la constante de equilibrio \\
& $K_{e q}^{\mu \sigma}$ & $K_{e q}^{\mu \sigma}$ \\
\hline \hline
\end{tabular}




\section{CAPÍTULO 4}

\section{Resultados y discusión sobre el ajuste de datos experimentales}

En este capítulo se presentan los resultados relacionados con los ajustes del modelo con datos experimentales reportados en la literatura. Se retoman los experimentos llevados a cabo por Sapei et al. (2012). Los investigadores estudiaron emulsiones dobles del tipo $\mathrm{W}_{1} / \mathrm{O} / \mathrm{W}_{2}$ que contenían cloruro de sodio $(\mathrm{NaCl})$ como soluto, y gelatina en la fase acuosa interna para aplicaciones de liberación controlada. Los experimentos fueron registrados aproximadamente por un mes y para diferentes concentraciones inicial de $\mathrm{NaCl}$ y de gelatina.

En los experimentos el tamaño medio de las gotas fue de 46 a 66 micrómetros de diámetro aproximadamente para el día 1, y se presentó la misma distribución para el día 29. Esto para el caso de los experimentos donde la concentración de $\mathrm{NaCl}$ fue variada y se mantuvo fija la concentración de gelatina. En los casos donde se mantuvo fija la concentración de $\mathrm{NaCl}$ en $2 \%$ y se cambió la concentración de gelatina, el tamaño medio de las gotas oscilo desde los 12 a los 42 micrómetros de diámetro aproximdamente en el primer día de experimentos y de 13 a 42 micrómetros para el último día (29) en que fueron monitoreadas las emulsiones.

En la Figura 14 se presentan los perfiles de concentración dentro de las gotas para diferentes tiempos de simulación. Además, se muestra el ajuste del porcentaje de soluto liberado con respecto a los datos experimentales cuando el porcentaje de $\mathrm{NaCl}$ es de $2 \%$ y de gelatina $3 \%$. En general se tiene un buen ajuste y el error aproximado se resume en la Tabla 3 (presentada más adelante). Para el caso que se presenta en la figura, el estado estacionario se alcanza aproximadamente a las 40 horas después de iniciado el experimento, lo cual se puede notar tanto en la cinética de liberación de soluto como en la concentración dentro de los glóbulos. Es de notar que los cambios significativos en la concentración ocurren en un tiempo corto de alrededor de $10 \mathrm{~h}$. 

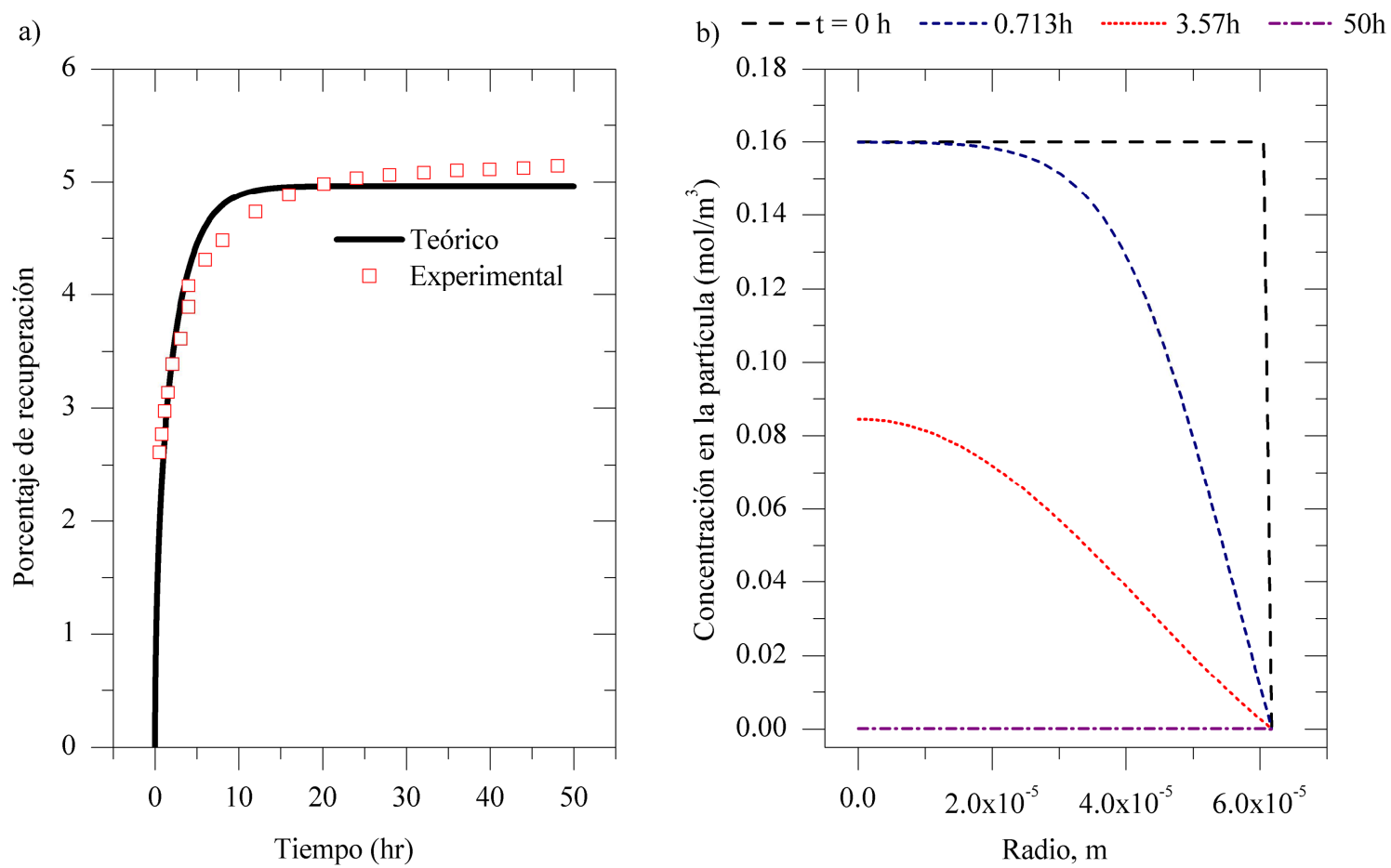

Figura 14. a) Perfiles de liberación de emulsiones dobles del tipo $\mathrm{W}_{1} / \mathrm{O} / \mathrm{W}_{2}$ con $2 \%$ de $\mathrm{NaCl}$, b) perfil de liberación de $\mathrm{NaCl}$ en la gota interior para diferentes tiempos.

En la Figura 15 se muestra el ajuste del porcentaje de soluto liberado con relación a los datos experimentales cuando el porcentaje de $\mathrm{NaCl}$ es de $4 \%$ y el de gelatina se mantiene constante en 3\%. Asimismo se presentan los perfiles de concentración dentro de las gotas para diferentes tiempos de simulación. El porcentaje de recuperación para este caso es del 5\%. El error aproximado se muestra en la Tabla 3. El estado estacionario para este caso, aproximadamente es alcanzado a las 40 horas después de que se inició el experimento, esto se puede apreciar en la cinética de liberación de soluto como en la concentración dentro de los glóbulos. Es necesario decir que los cambios significativos en la concentración ocurren a un tiempo corto de alrededor de 10 horas. En general se tiene un buen ajuste del modelo. 

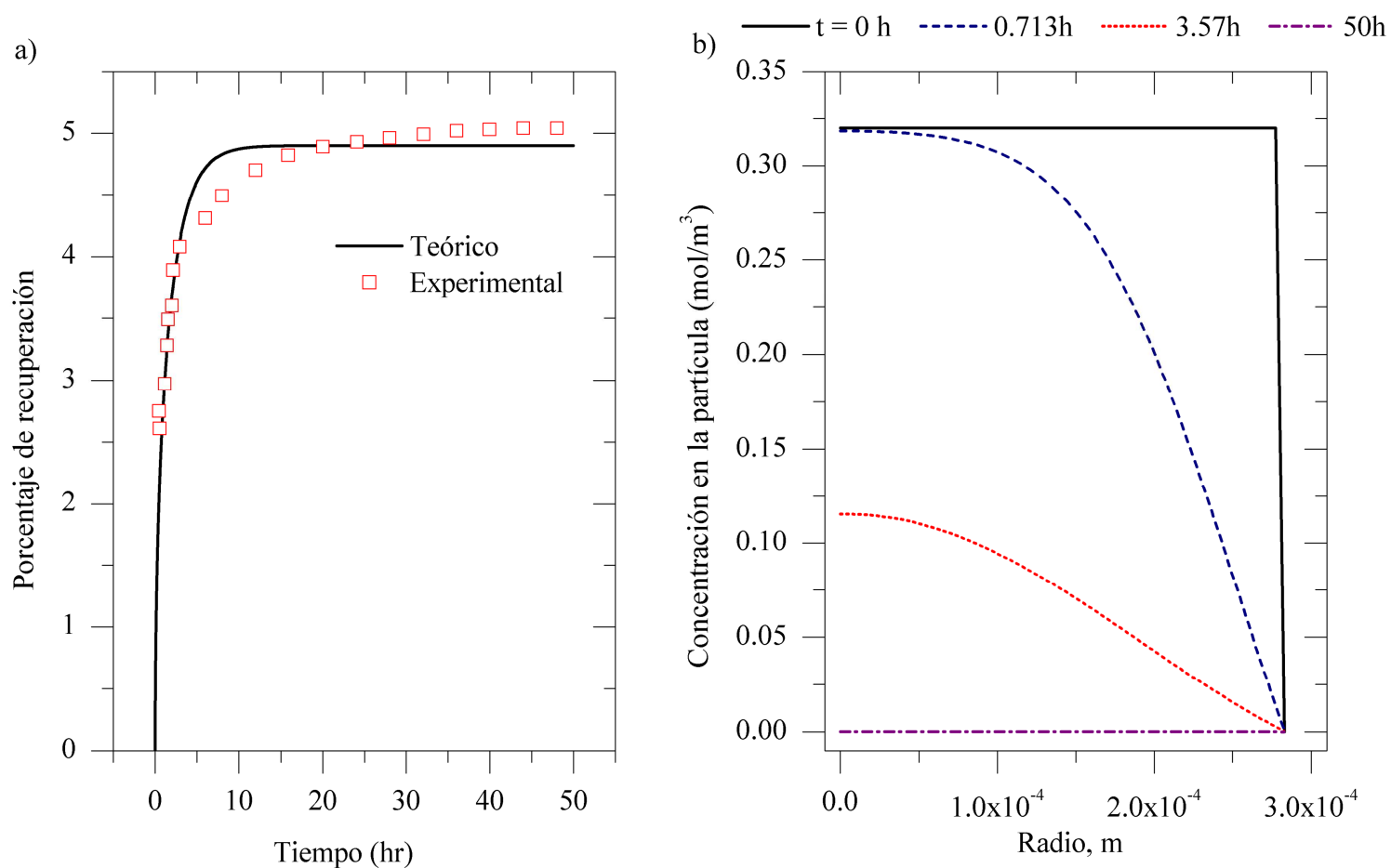

Figura 15. a) Perfiles de liberación de emulsiones del tipo $\mathrm{W}_{1} / \mathrm{O} / \mathrm{W}_{2}$ con $4 \%$ de $\mathrm{NaCl}$, b) Perfil de liberación de $\mathrm{NaCl}$ en la gota interior para diferentes tiempos.

El estado estacionario que se alcanza en la Figura 16 es de 40 horas después de iniciado el experimento, se puede apreciar en la concentración dentro de los glóbulos y en la cinética de liberación del soluto. Por otro lado los cambios significativos que se dan en la concentración suceden en un tiempo corto de aproximadamente 10 horas. Se puede decir que se tiene un buen ajuste del modelo con los datos experimentales, el error aproximado se aprecia en la Tabla 3. Para el caso que se presenta en la figura el ajuste del porcentaje de soluto liberado con relación a los datos experimentales cuando el porcentaje de gelatina es de $3 \%$ y el de $\mathrm{NaCl}$ es de $6 \%$. También se muestran los perfiles de concentración dentro de las gotas para diferentes tiempos de simulación. En este caso el porcentaje de recuperación es de aproximadamente $4.8 \%$. 

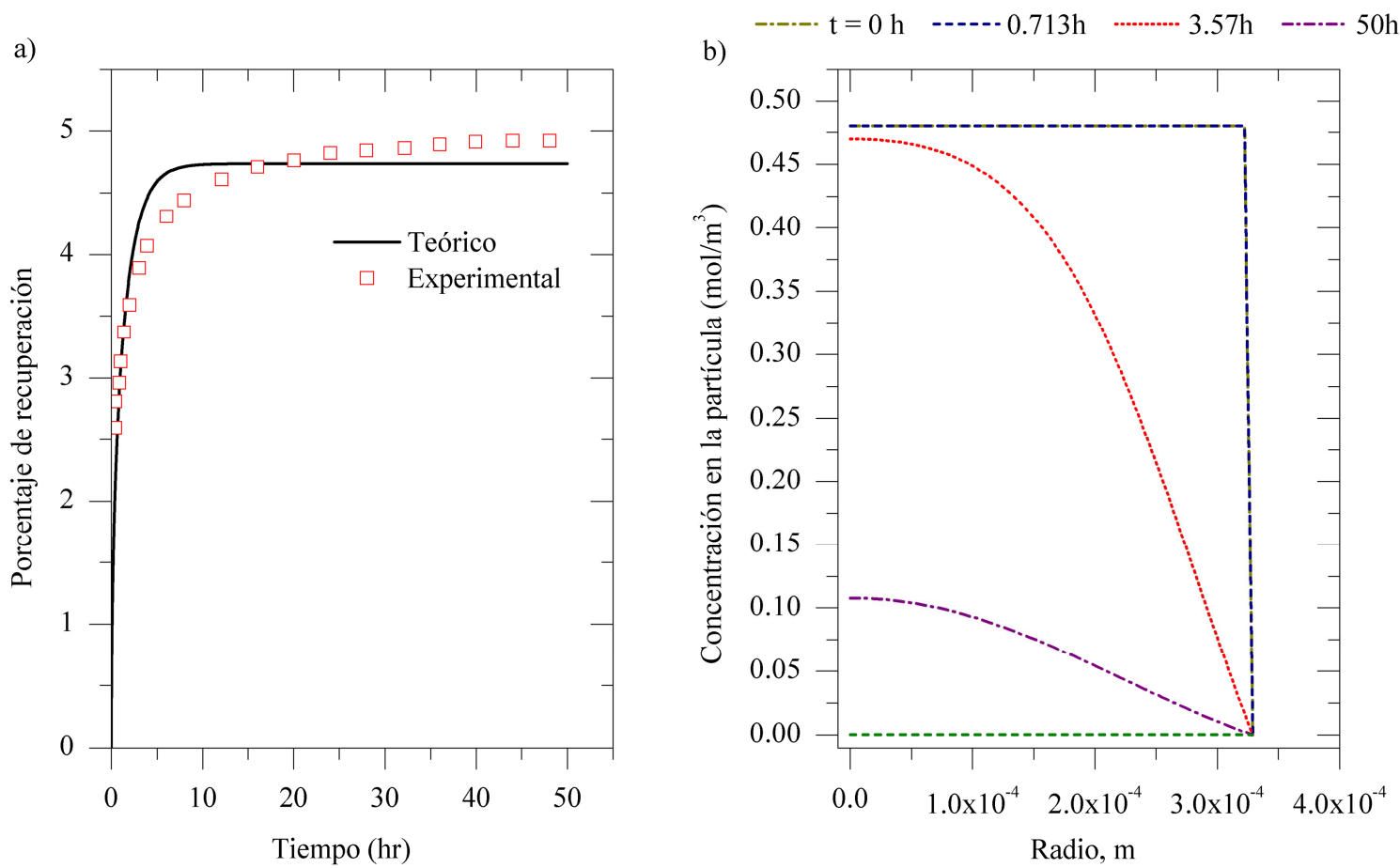

Figura 16. a) Perfiles de liberación de emulsión doble del tipo $\mathrm{W}_{1} / \mathrm{O} / \mathrm{W}_{2}$ con $6 \%$ de $\mathrm{NaCl}$, b) Perfil de liberación de $\mathrm{NaCl}$ de la gota interior para diferentes tiempos.

En la Figura 17 se observa el ajuste del porcentaje de soluto liberado con relación a los datos experimentales, cuando el porcentaje de $\mathrm{NaCl}$ es de $8 \%$ y el de gelatina como ya se mencionó en las figuras anteriores se mantiene constante en $3 \%$. Se aprecia también los perfiles de concentración en las gotas internas para diferentes tiempos de simulación. El porcentaje de recuperación en este caso es de $4.5 \%$. Los cambios significativos que se dan en la concentración suceden en un tiempo aproximado de 10 horas, lo cual indica que se dan en un tiempo corto. En este caso el estado estacionario se alcanza 40 horas después de que se inició el experimento, esto se observa en la concentración dentro de los glóbulos y en la cinética de liberación del soluto. En general el ajuste del modelo es bueno. El error aproximado que se obtuvo para este caso se aprecia en la Tabla 3. 

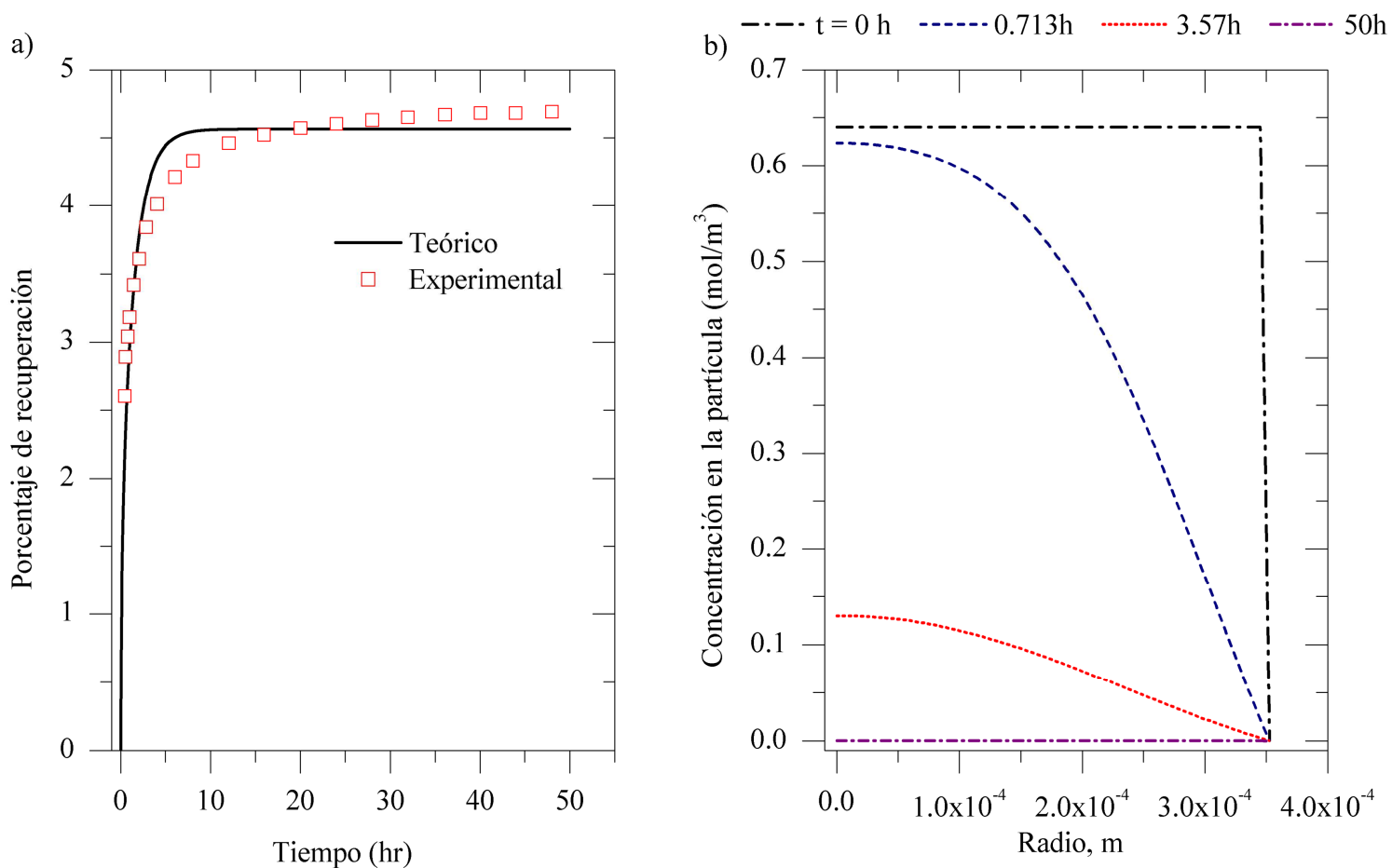

Figura 17. a) Perfil de liberación de emulsión doble del tipo $\mathrm{W}_{1} / \mathrm{O} / \mathrm{W}_{2}$ con $8 \%$ de $\mathrm{NaCl}$, b) Perfil de liberación de $\mathrm{NaCl}$ en la gota interna para diferentes tiempos.

En las Figuras 18-20 se grafican los datos experimentales de Sapei et al. (2012) y las predicciones del modelo teórico para la liberación de sal $(\mathrm{NaCl})$ en las emulsiones dobles conteniendo $2 \%$ de sal $(\mathrm{NaCl})$ en la fase acuosa interna y estabilizadas con diferentes concentraciones de gelatina. El ajuste del porcentaje de soluto liberado con relación a los datos experimentales cuando el porcentaje de $\mathrm{NaCl}$ se mantiene constante en $2 \%$ y el de gelatina es de $3 \%$, se muestra en la Figura 18, donde también se observan los perfiles de concentración dentro de las gotas para diferentes tiempos de simulación. En general el ajuste del modelo es bueno, y el error aproximado que se obtuvo se resume en la Tabla 3. En este caso el estado estacionario se alcanza a las 40 horas aproximadamente después de que se inició el experimento, esto se muestra en la concentración dentro de los glóbulos y en la cinética de liberación del soluto. Los cambios significativos que se dan en la concentración suceden en un tiempo corto de aproximadamente 10 horas, y el porcentaje de recuperación es de alrededor de $4.5 \%$ según las predicciones teóricas. Con los datos 
experimentales el porcentaje de liberación es casi del $5 \%$, aunque en este caso no es posible estimar con precisión el tiempo en el que se alcanza el estado estacionario, por lo que es importante mencionar que se necesita más tiempo de monitoreo para las emulsiones aquí estudiadas.

a)

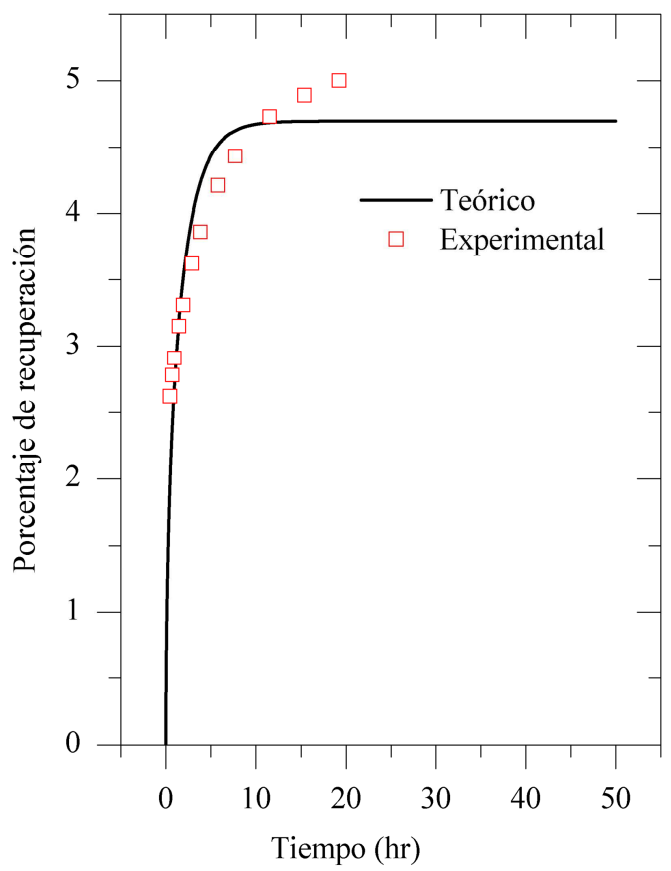

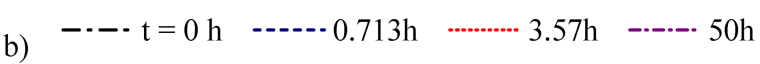

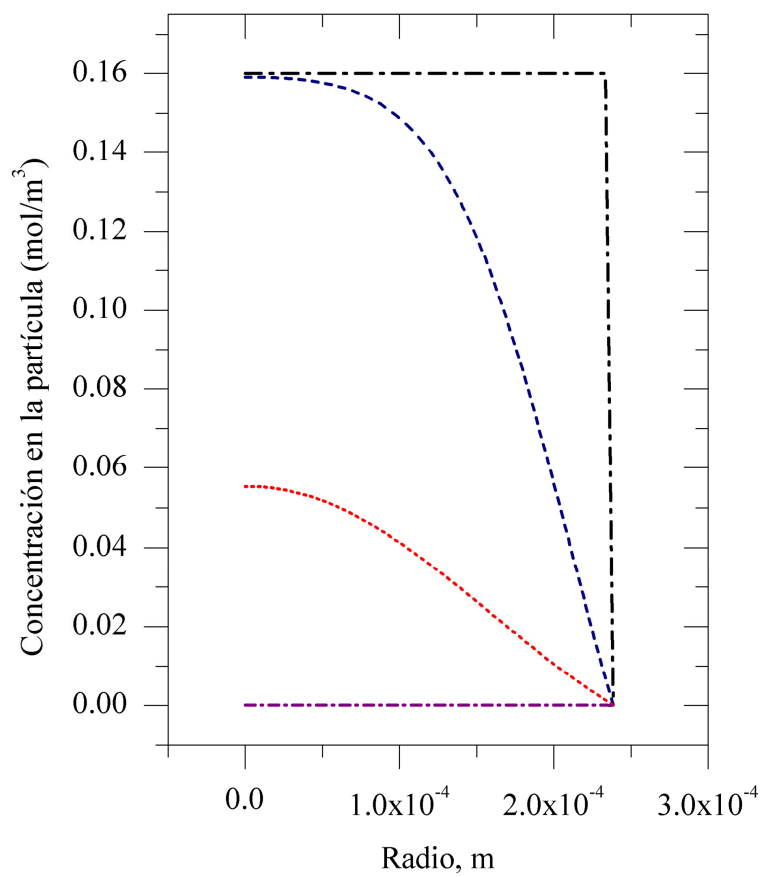

Figura 18. Perfiles de liberación de emulsiones dobles del tipo $W_{1} / O / W_{2}$ con $3 \%$ de gelatina, b) Perfil de liberación de $\mathrm{NaCl}$ de la gota interna para diferentes tiempos.

La Figura 19 muestra los perfiles de concentración dentro de las gotas para diferentes tiempos de simulación, y el ajuste del porcentaje de soluto liberado con relación a los datos experimentales cuando el porcentaje de gelatina es de $10 \%$ y el de $\mathrm{NaCl}$ es de $2 \%$. El error aproximado se resume en la Tabla 3. El ajuste para este caso es bueno, y el estado estacionario se alcanza 30 horas después de iniciado el experimento, esto se observa en la concentración dentro de los glóbulos y en la cinética de liberación del soluto. Los cambios significativos se dan en un tiempo relativamente corto, aproximadamente 10 horas, el 
porcentaje de recuperación es de cerca del $3.8 \%$, al igual que en la figura anterior los datos experimentales no permiten observar con claridad donde se alcanza el estado estacionario, ya que hacen falta más puntos experimentales o un monitoreo por más tiempo
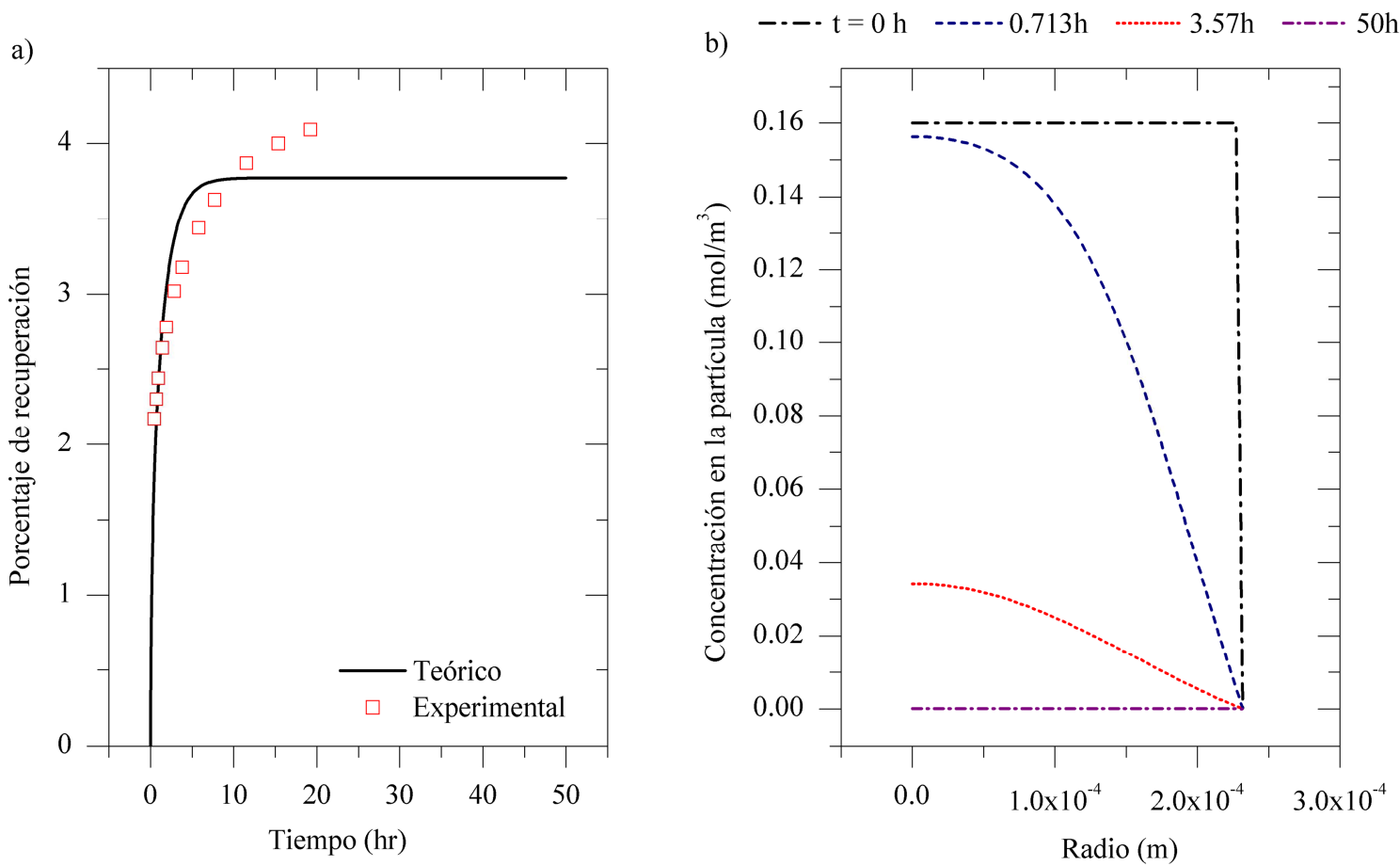

Figura 19. Perfiles de liberación de emulsiones dobles del tipo $W_{1} / O / W_{2}$ con $10 \%$ de gelatina, b) Perfil de liberación de $\mathrm{NaCl}$ para diferentes tiempos.

En la Figura 20 se presentan los perfiles de concentración dentro de las gotas para diferentes tiempos de simulación. Además se muestra el ajuste del porcentaje de soluto liberado con respecto a los datos experimentales cuando el porcentaje de $\mathrm{NaCl}$ es de $2 \%$ y de gelatina $10 \%$. En general se tiene un buen ajuste y el error aproximado se resume en la Tabla 3. Para el caso que se presenta en la figura, el estado estacionario se alcanza aproximadamente a las 40 horas después de iniciado el experimento, lo cual se puede notar tanto en la cinética de liberación de soluto como en la concentración dentro de los glóbulos. Es de notar que los cambios significativos en la concentración ocurren en un tiempo corto de alrededor de $10 \mathrm{~h}$. 
El porcentaje de recuperación que se calcula con el modelo para este caso es de aproximadamente $5.5 \%$. Una vez más es importante señalar que los datos experimentales aún están se encuentran en la etapa de transición temporal y aún se alcanza el estado estacionario. Esto dificulta el análisis teórico y no se puede hacer un ajuste más preciso con los datos experimentales.
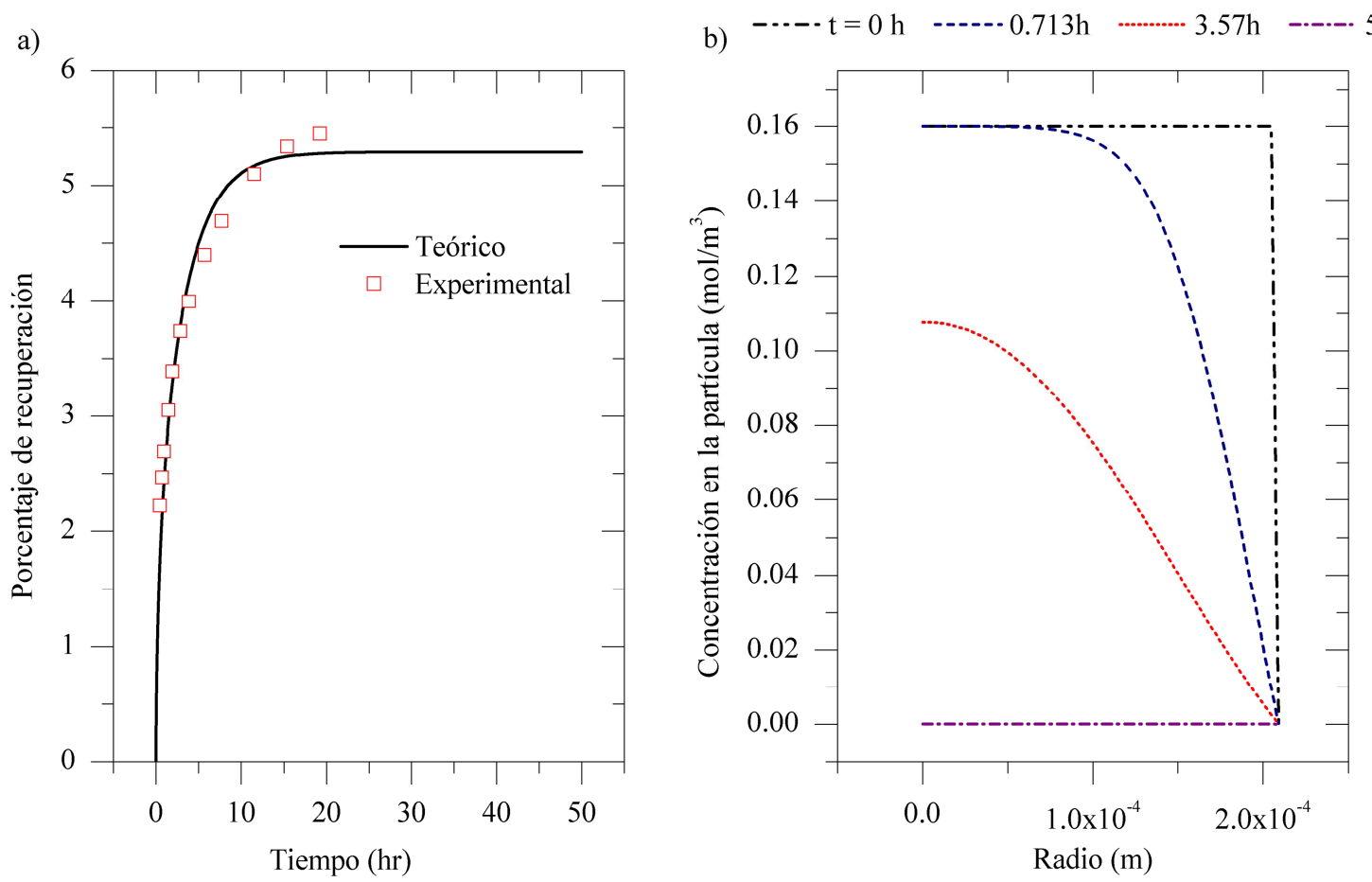

Figura 20. Perfiles de liberación de emulsiones dobles del tipo $W_{1} / O / W_{2}$ con $0 \%$ de gelatina, b) Perfil de liberación de $\mathrm{NaCl}$ de la gota interna para diferentes tiempos

A continuación se retoman los experimentos llevados a cabo por Lutz et al (2009). Los investigadores estudiaron emulsiones dobles del tipo $\mathrm{W}_{1} / \mathrm{O} / \mathrm{W}_{2}$ que contenían cloruro de sodio $(\mathrm{NaCl})$ como soluto, y usando en la fase oleosa diferentes aceites como el $\mathrm{R}(+)$ Limoneno y MCT (triglicéridos de cadena media) para aplicaciones de liberación controlada. Los experimentos fueron registrados aproximadamente por un mes y para diferentes concentraciones iniciales de $\mathrm{NaCl}$.

Es importante resaltar que los investigadores utilizaron para la preparación de las emulsiones dobles diferentes agentes estabilizantes como el WPI/C63 (aislado de proteína) 
y el WPI/U63. Se resalta lo anterior para justificar los perfiles de concentración donde se utiliza el mismo porcentaje de $\mathrm{NaCl}(4.4 \%)$.

Se presentan en la Figura 21 los perfiles de concentración dentro de las gotas para diferentes tiempos de simulación. Además se muestra el ajuste del porcentaje de soluto liberado con respecto a los datos experimentales cuando el porcentaje de $\mathrm{NaCl}$ es de $4.4 \%$, utilizando en la fase oleosa R(+)-Limoneno y como agente estabilizador el WPI/U63. En general se tiene un buen ajuste y el error aproximado se presenta en la Tabla 3. Para el caso que se presenta en la figura, el estado estacionario se alcanza aproximadamente a las 800 horas después de iniciado el experimento, lo cual se puede notar tanto en la cinética de liberación de soluto como en la concentración dentro de los glóbulos. Es de notar que los cambios significativos en la concentración ocurren en un tiempo de alrededor de $700 \mathrm{~h}$.

Los datos experimentales muestran que se requiere de más tiempo para alcanzar el estado estacionario ya que se observa que la $\mathrm{NaCl}$ no se ha liberado completamente.
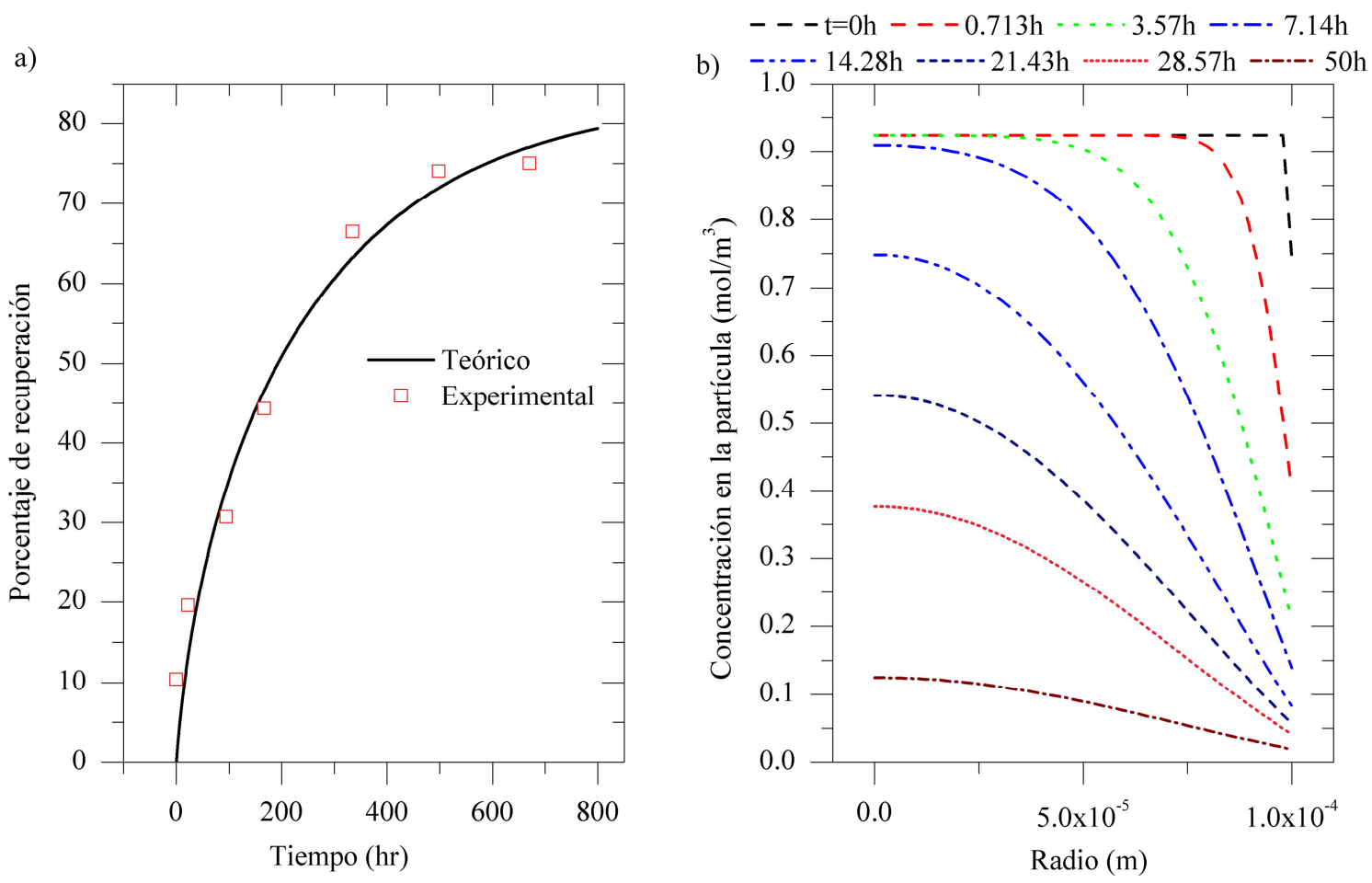

Figura 21. a) Perfil de liberación (\%) de $\mathrm{NaCl}$, para una concentración de $4.4 \%$ de NaCl, estabilizada con WPI, b) Perfil de liberación de $\mathrm{NaCl}$ de la partícula interna para diferentes tiempos. 
El ajuste del porcentaje de soluto liberado con respecto a los datos experimentales cuando el porcentaje de $\mathrm{NaCl}$ es de $1 \%$, utilizando el aceite MCT y como agente estabilizador WPI/C63 se presenta en la Figura 22. Además, se presentan los perfiles de concentración dentro de las gotas para diferentes tiempos de simulación. Se observa un buen ajuste del modelo y el error aproximado se resume en la Tabla 3. Para el caso que se presenta en la figura, el estado estacionario se alcanza aproximadamente a las 800 horas después de iniciado el experimento, lo cual se puede notar tanto en la cinética de liberación de soluto como en la concentración dentro de los glóbulos. Cabe resaltar que no se puede estimar con precisión a que tiempo se presentan los cambios significativos en la concentración. Esto debido a que no hay suficientes datos experimentales, lo cual se nota en el lado b) de la figura ya que se observa que la $\mathrm{NaCl}$ no se ha liberado completamente. Mientras tanto, en el lado a) de dicha figura se muestra que el porcentaje de recuperación es cercana al $100 \%$.
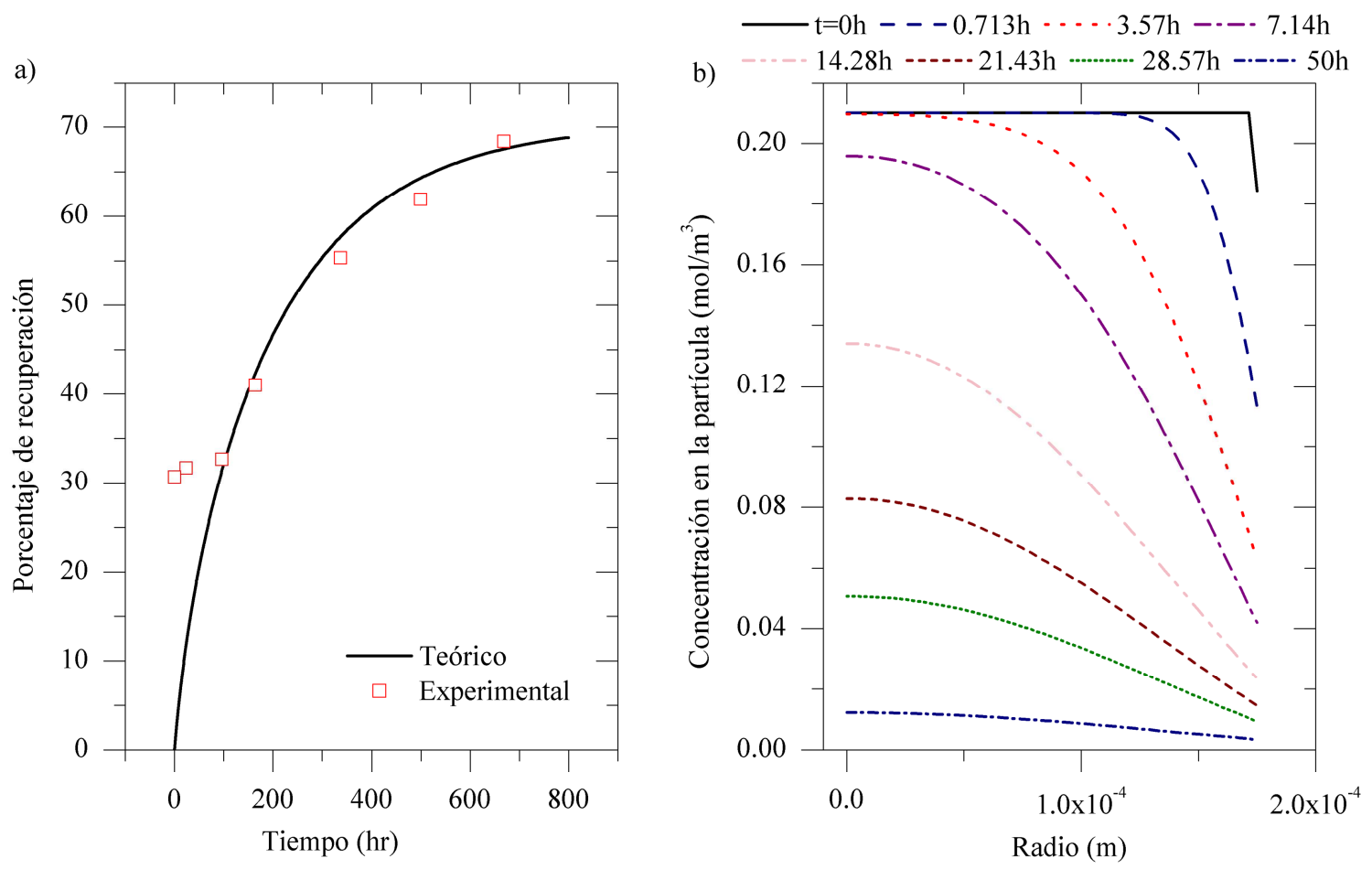

Figura 22. Perfil de liberación de $\mathrm{NaCl}$, para una concentración de $1 \%$ de $\mathrm{NaCl}$, estabilizada con WPI, b) Perfil de liberación de $\mathrm{NaCl}$ de la partícula interna para diferentes tiempos. 
La Figura 23 presenta el ajuste del porcentaje de soluto liberado con respecto a los datos experimentales cuando el porcentaje de $\mathrm{NaCl}$ es de $1.5 \%$, utilizando MCT como fase oleica y WPI/C63 como agente estabilizador. También se presentan los perfiles de concentración dentro de las gotas para diferentes tiempos de simulación. Se observa que se tiene un buen ajuste, el error aproximado se resume en la Tabla 3. Para el caso que se presenta en la figura, el estado estacionario se alcanza aproximadamente a las 750 horas después de iniciado el experimento, lo cual se puede notar tanto en la cinética de liberación de soluto como en la concentración dentro de los glóbulos.

En el lado b) de la figura se observa que la liberación de $\mathrm{NaCl}$ no se ha llevado a cabo completamente, sin embargo se ha liberado la mayoría de soluto por lo que el tiempo cuando suceden cambios significativos es de aproximadamente 600 horas. Además, en el lado a) se muestra que el porcentaje de liberación es cerca del $50 \%$.

a)

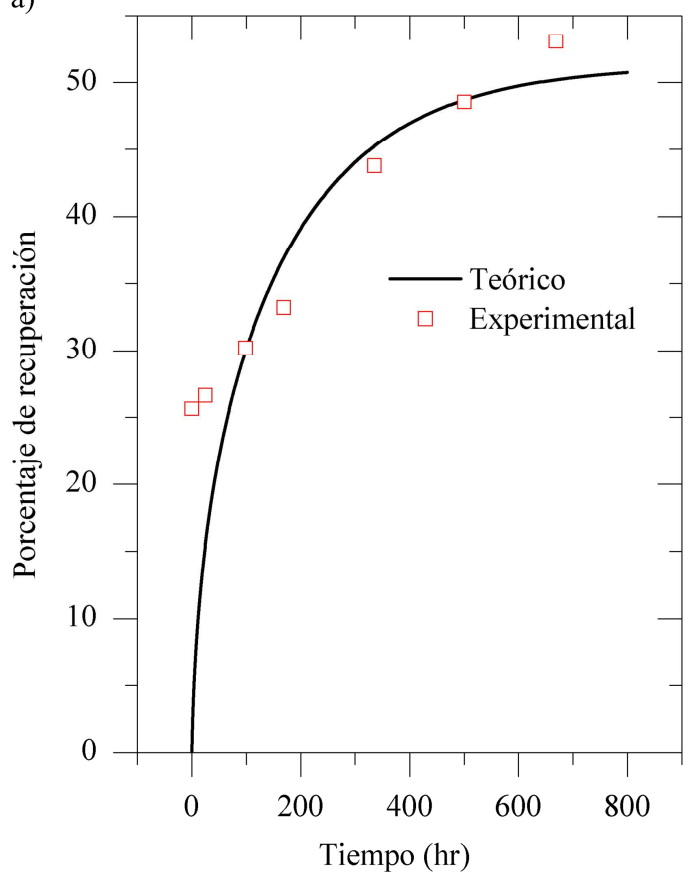

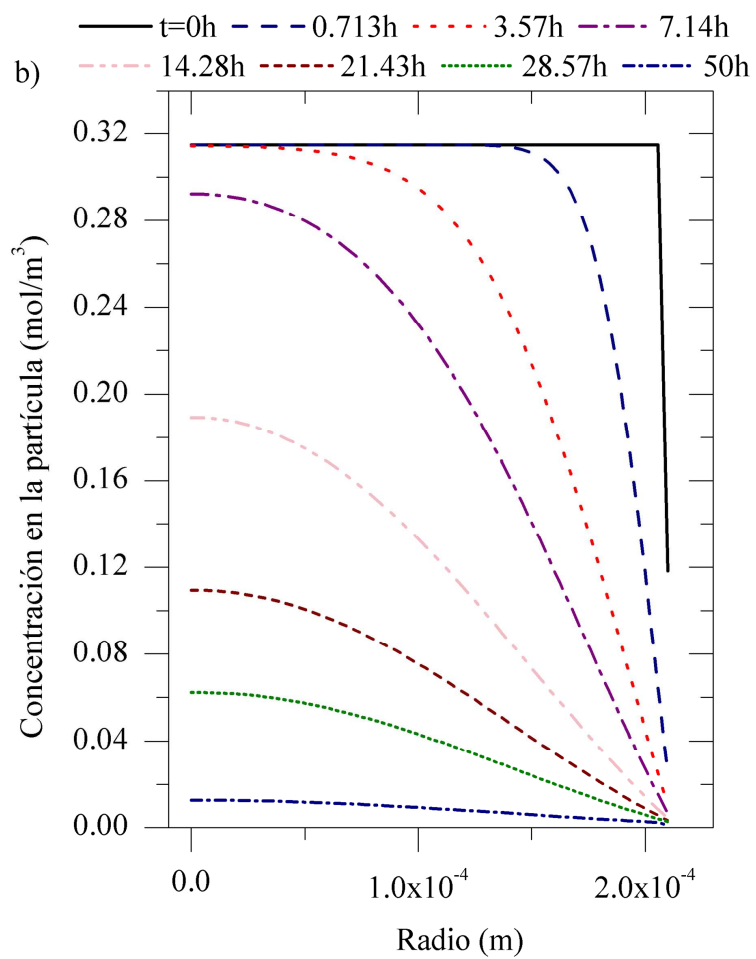

Figura 23. Perfil de liberación de $\mathrm{NaCl}$, para una concentración de $1.5 \%$ de $\mathrm{NaCl}$, estabilizada con WPI, b) Perfil de liberación de $\mathrm{NaCl}$ de la partícula interna para diferentes tiempos 
En la Figura 24 se presenta el ajuste del porcentaje de soluto liberado con respecto a los datos experimentales cuando el porcentaje de $\mathrm{NaCl}$ es de $4.4 \%$ utilizando MCT como fase oleosa MCT y WPI/C63 como agente estabilizador. También se presentan los perfiles de concentración dentro de las gotas para diferentes tiempos de simulación. Se observa que se tiene un buen ajuste, el error aproximado se resume en la Tabla 3. En el lado a) de la figura es difícil estimar el tiempo al que se alcanzara el estado estacionario, esto debido a la falta de tiempo al monitorear el sistema experimental. No obstante, en el lado b) de la figura muestra que prácticamente se ha liberado el soluto.
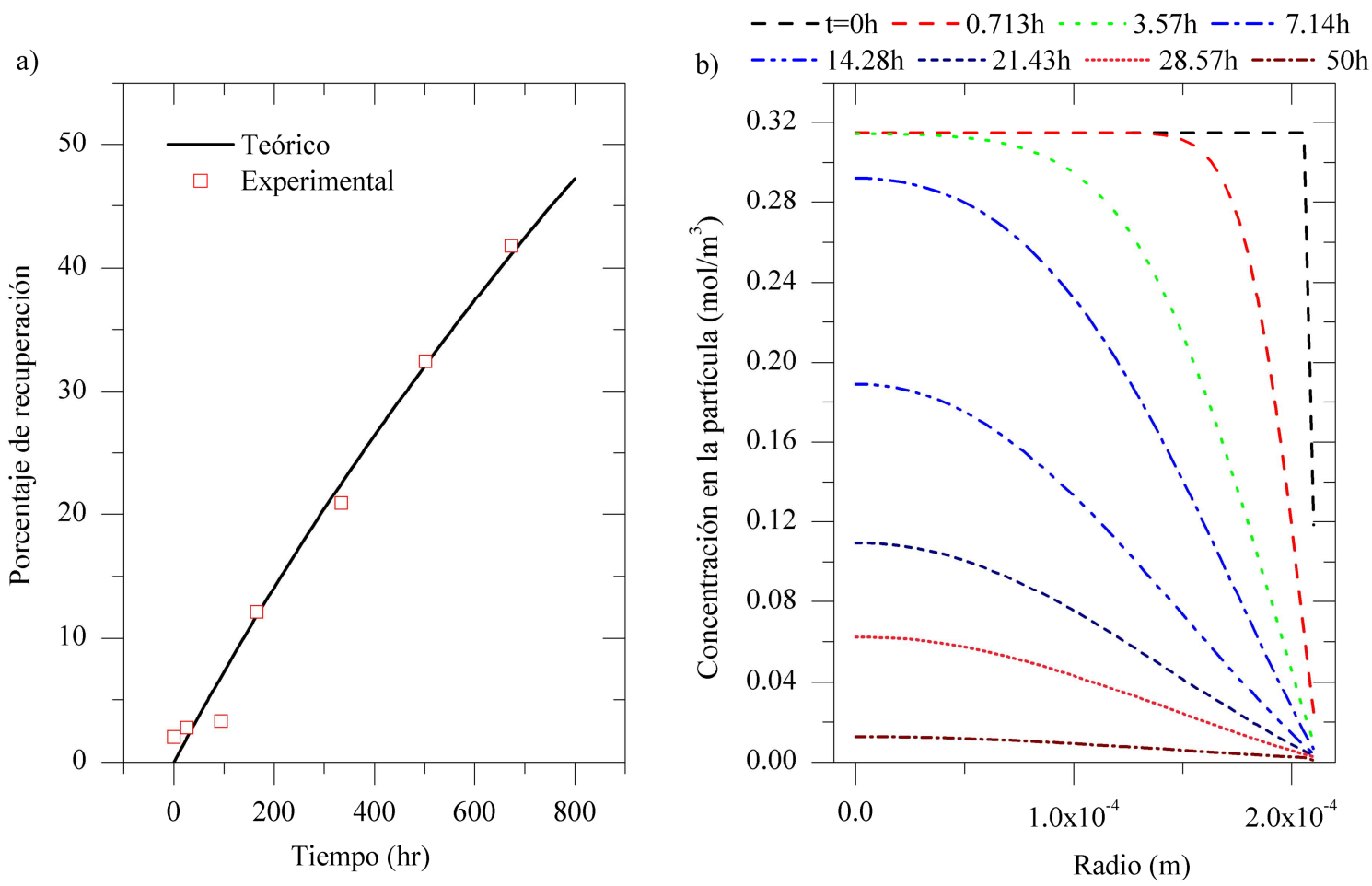

Figura 24. Perfil de liberación de $\mathrm{NaCl}$, para una concentración de $4.4 \%$ de $\mathrm{NaCl}$, estabilizada con WPI, b) Perfil de liberación de $\mathrm{NaCl}$ de la partícula interna para diferentes tiempos

En la Figura 25 se presentan los perfiles de concentración dentro de las gotas para diferentes tiempos de simulación, así como el ajuste del porcentaje de soluto liberado con respecto a los datos experimentales, cuando el porcentaje de $\mathrm{NaCl}$ es de $4.4 \%$ utilizando 
como aceite en la fase oleosa MCT y como agente estabilizador WPI/C63. Por lo general el ajuste del modelo es aceptable, y el error encontrado para este caso se presenta en la Tabla 3. Como comentario adicional, al igual que en la figura anterior, se hace hincapié en la falta de más datos experimentales, esto con la finalidad de tener una mayor certeza del tiempo en el que se alcanza el estado estacionario. Aunque para este caso en particular en el lado b) de la figura se observa que el soluto no se ha liberado completamente. Esto debido a la falta de tiempo en el desarrollo experimental. A diferencia de la figura anterior la liberación de $\mathrm{NaCl}$ es lenta.
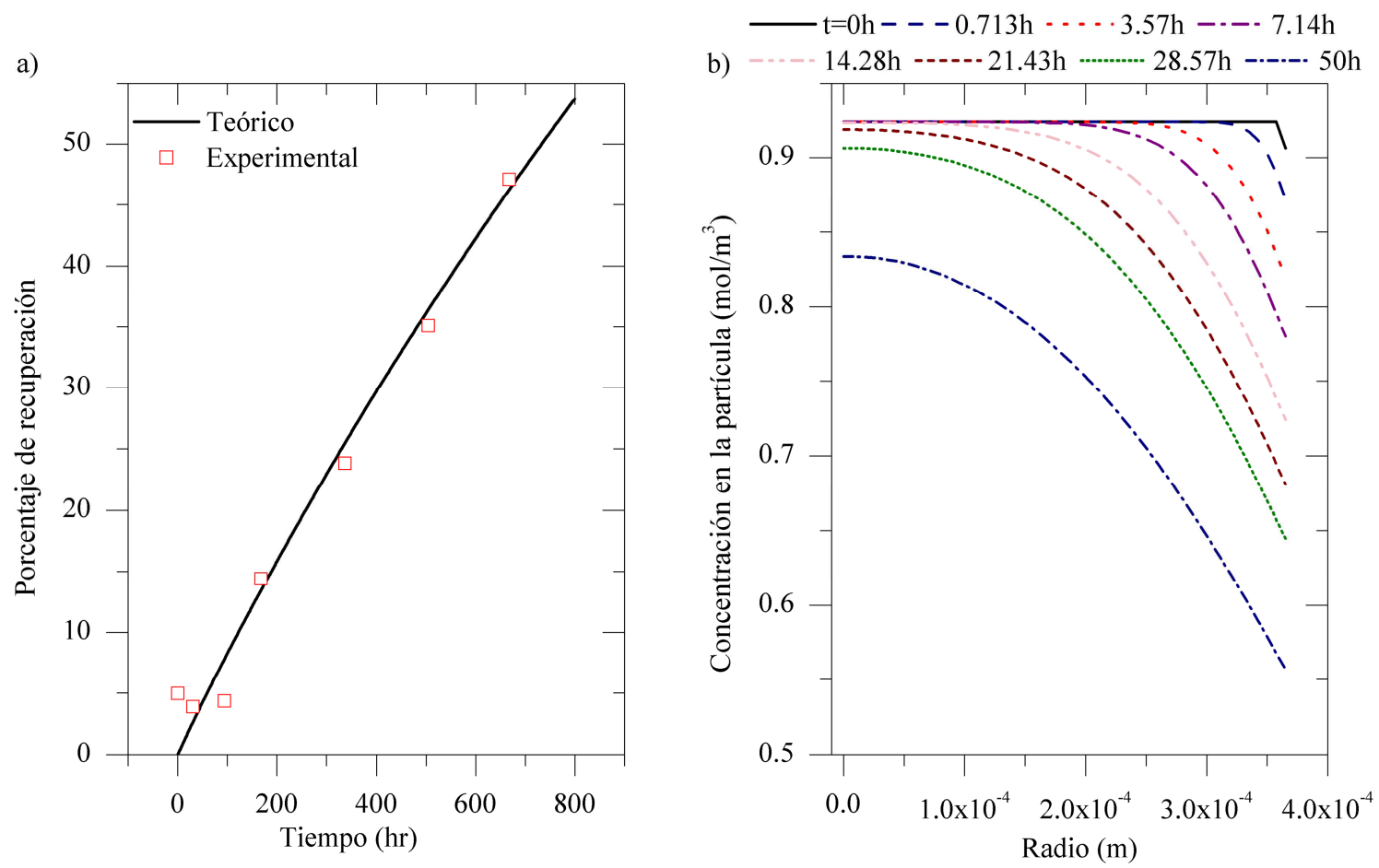

Figura 25. Perfil de liberación de $\mathrm{NaCl}$, para una concentración de $4.4 \%$ de $\mathrm{NaCl}$, estabilizada con WPI/C63, b) Perfil de liberación de $\mathrm{NaCl}$ de la partícula interna para diferentes tiempos.

La Figura 26 presenta los perfiles de concentración de las gotas para diferentes tiempos de simulación, y el ajuste del porcentaje de soluto liberado con respecto a los datos experimentales cuando el porcentaje de $\mathrm{NaCl}$ es de $4.4 \%$. La emulsión doble se estabilizó 
con WPI/U63 y para la fase oleosa se utilizó MCT. El error encontrado se presenta en la Tabla 3. En general el ajuste del modelo es bueno. Se observa que igual que en figuras anteriores los datos experimentales no son suficientes para mostrar el tiempo en el que se alcanza el estado estacionario. Esto se complementa con los datos graficados en el lado b) de la Figura 26, donde se nota una liberación lenta del soluto.
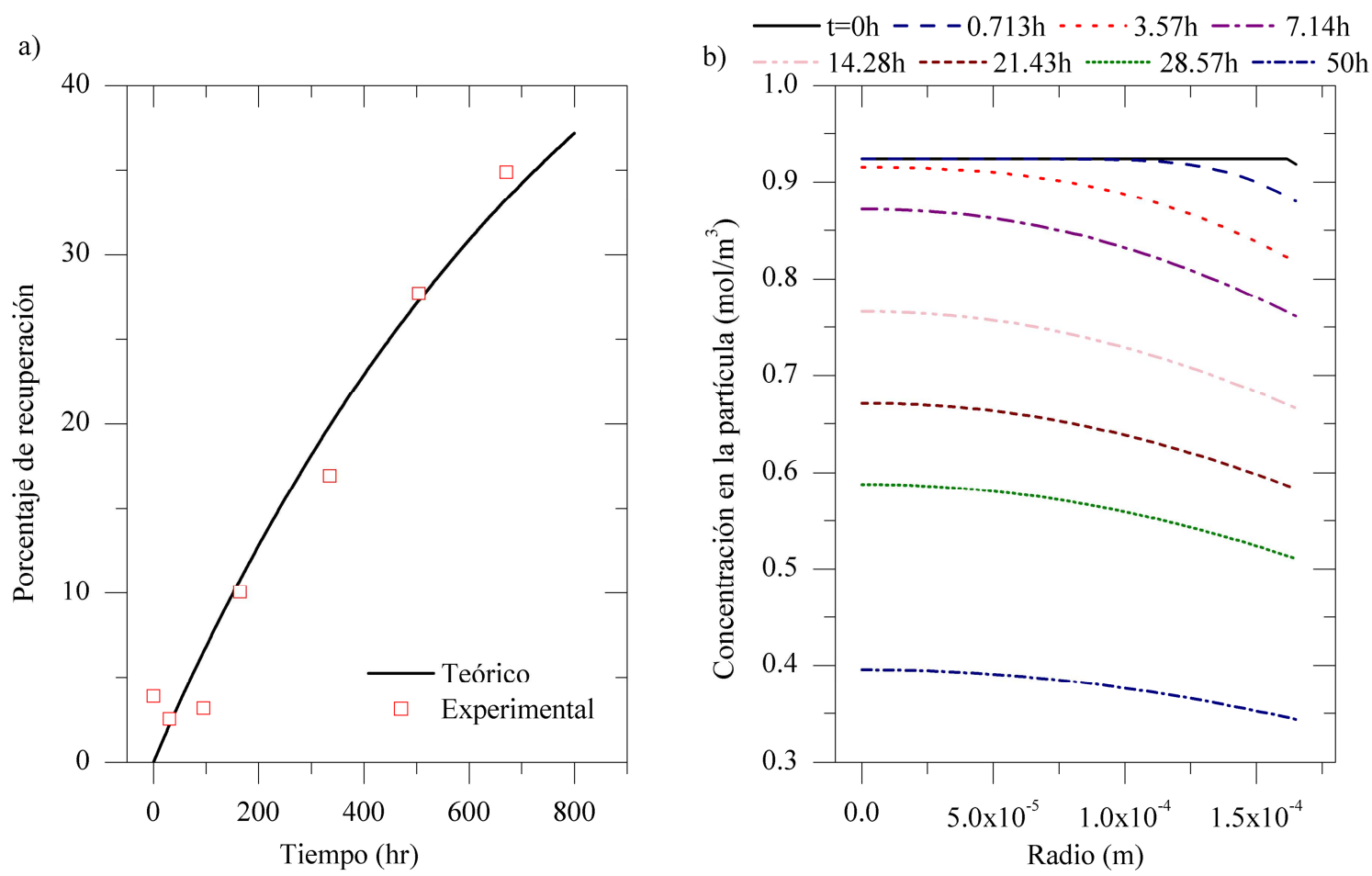

Figura 26. Perfil de liberación de $\mathrm{NaCl}$, para una concentración de $4.4 \%$ de $\mathrm{NaCl}$, estabilizada con WPI/U63, b) Perfil de liberación de $\mathrm{NaCl}$ de la partícula interna para diferentes tiempos.

Finalmente, en la Tabla 3 se resumen diversas cantidades calculadas durante el ajuste de datos experimentales como son: el coeficiente de equilibrio, la permeabilidad efectiva, la difusividad efectiva y el error del ajuste. El error se definió como:

$$
\text { Error }=\sum_{n}\left(\psi_{n}^{\mathrm{exp}}-\psi_{n}^{\mathrm{teo}}\right)^{2}
$$


Donde $n$ es el índice del dato experimental, $\psi_{n}^{\exp }$ representa cualquier cantidad experimental que va a ser ajustada como la concentración o el porcentaje de soluto liberado, mientras que $\psi_{n}^{\text {teo }}$ es la cantidad análoga calculada con el modelo teórico. Para encontrar los valores óptimos de las constantes reportadas en la Tabla 3 se implementó una rutina en Matlab para minimizar el error. De esta forma, los datos reportados corresponden al mínimo del error definido en (94). El método de solución del modelo por diferencias finitas y el procedimiento para minimizar el error se presentan en el Apéndice A.

La rapidez de difusión del soluto $(\mathrm{NaCl})$ dentro de los glóbulos se cuantifica principalmente a través del coeficiente de difusión efectiva $D_{\omega}$. De los resultados reportados en la Tabla 3 se concluye que para obtener mejor transferencia de soluto dentro del sistema de emulsión doble es recomendable utilizar aceite de canola con gelatina como agente tensoactivo y altas concentraciones de soluto.

La facilidad que presenta cierto soluto a pasar a través de la interfaz de los glóbulos más grandes hacia el líquido exterior es cuantificada con el producto del coeficiente de permeabilidad efectiva $a_{\eta \omega} P_{e f f}^{\eta}$. Dicho coeficiente presenta gran variedad en los ajustes realizados, pero en general se puede concluir que los sistemas de emulsión doble con valores más grandes de este coeficiente son aquellos conformados por: aceite de canola con gelatina y altas concentraciones de soluto. Esta combinación también maximiza el valor de $D_{\omega}$, por lo que se discutió en el párrafo anterior. De lo cual se deduce que, posiblemente, altas tasas de liberación de soluto mejoran el transporte interno dentro del sistema de emulsión. Lo cual no necesariamente es cierto, dado que, por ejemplo, teóricamente puede haber sistemas con difusión interna alta, pero bajo intercambio de soluto en la interfaz, es decir, existir un cuello de botella en las fronteras del sistema.

Ahora, el tiempo requerido para alcanzar el estado estable en el sistema puede cuantificarse con el coeficiente de equilibrio $K_{e q}^{\mu \sigma}$. Evidentemente, altos valores del coeficiente indican que el sistema de emulsión doble requiere de más tiempo para estabilizarse (considerando las velocidades de liberación de soluto). Los sistemas con más altos valores del coeficiente 
de equilibrio son los que utilizan $\mathrm{R}(+)$-Limoneno y MCT como aceite, y los más bajos corresponden a los de aceite de canola.

La utilidad de los resultados resumidos en la Tabla 3 radica en ofrecer una rápida configuración del sistema de emulsión doble de acuerdo a las características deseadas de liberación de soluto. Para liberación de $\mathrm{NaCl}$, una rápida liberación se obtiene utilizando aceite de canola con gelatina como agente tensoactivo. Mientras que para una liberación más prolongada entonces es recomendable utilizar MCT o $\mathrm{R}(+)$-Limoneno como fase oleica combinada con WPI como agente tensoactivo. Evidentemente, este tipo de análisis puede extenderse a otros sistemas de emulsiones dobles reportados en la literatura.

Cabe señalar que se encontraron dificultades en los ajustes para sistemas que utilizan MCT como fase oleica, debido a la falta de más datos experimentales para definir con mayor precisión el tiempo mínimo para alcanzar el estado estacionario. Esto puede observarse con los errores reportados en la Tabla 3.

Tabla 3. Parámetros estimados y errores calculados.

\begin{tabular}{|c|c|c|c|c|c|c|}
\hline $\begin{array}{c}\text { Tipo de } \\
\text { aceite }\end{array}$ & $\begin{array}{c}\text { Agente } \\
\text { tensoactivo }\end{array}$ & $\begin{array}{c}\text { Concentraci } \\
\text { ón de NaCl } \\
{[\%]}\end{array}$ & $K_{e q}^{\mu \sigma}$ & $\begin{array}{c}a_{\eta \omega} P_{e f f}^{\eta} \\
{[1 / \mathrm{s}]}\end{array}$ & $D_{\omega}\left[\mathrm{m}^{2} / \mathrm{s}\right]$ & Error \\
\hline Canola & $\begin{array}{c}3 \% \text { de } \\
\text { gelatina }\end{array}$ & 2 & 92 & 0.92 & $1.19 \times 10^{-12}$ & 1.24 \\
\hline Canola & $\begin{array}{c}3 \% \text { de } \\
\text { gelatina }\end{array}$ & 4 & 18.2 & 47.32 & $7 \times 10^{-12}$ & 1.21 \\
\hline Canola & $\begin{array}{c}3 \% \text { de } \\
\text { gelatina }\end{array}$ & 6 & 14.8 & 47.36 & $1.01 \times 10^{-11}$ & 1.21 \\
\hline Canola & $\begin{array}{c}3 \% \text { de } \\
\text { gelatina }\end{array}$ & 8 & 13.1 & 95.63 & $1.09 \times 10^{-11}$ & 1.05 \\
\hline Canola & $\begin{array}{c}0 \% \text { de } \\
\text { gelatina }\end{array}$ & 2 & 27.6 & 27.6 & $3.4 \times 10^{-12}$ & 0.58 \\
\hline Canola & $\begin{array}{c}3 \% \text { de } \\
\text { gelatina }\end{array}$ & 2 & 21 & 12.6 & $5.8 \times 10^{-12}$ & 1.08 \\
\hline
\end{tabular}




\begin{tabular}{|c|c|c|c|c|c|c|}
\hline Canola & $\begin{array}{c}10 \% \text { de } \\
\text { gelatina }\end{array}$ & 2 & 17 & 10.2 & $5.8 \times 10^{-12}$ & 0.75 \\
\hline MCT & WPI & 1 & 480 & $3.12 \times 10^{-8}$ & $8 \times 10^{-13}$ & 1357 \\
\hline MCT & WPI & 1.5 & 290 & $2.33 \times 10^{-8}$ & $6 \times 10^{-13}$ & 806 \\
\hline MCT & WPI/C63 & 4.4 & $1.05 \times 10^{-2}$ & $5.2 \times 10^{-6}$ & $1 \times 10^{-14}$ & 21.43 \\
\hline $\begin{array}{c}\text { R(+)- } \\
\text { Limoneno }\end{array}$ & WPI & 4.4 & 1000 & $4.4 \times 10^{-8}$ & $3.6 \times 10^{-13}$ & 190 \\
\hline MCT & WPI/C63 & 4.4 & 640 & $3.2 \times 10^{-9}$ & $1.2 \times 10^{-12}$ & 43.85 \\
\hline MCT & WPI/U63 & 4.4 & 440 & $2.64 \times 10^{-9}$ & $1.5 \times 10^{-12}$ & 38.44 \\
\hline
\end{tabular}




\section{Conclusiones}

En este trabajo se utilizó el modelo de Morales-Zárate et al. (2008) para el modelado del transporte de soluto en sistemas de emulsión doble. Utilizando el método de diferencias finitas, se discretizó y resolvió el modelo en un código en Matlab. Se encontró que el esquema discretizado es estable y se tienen resultados consistentes (independientes del número de nodos) cuando los coeficientes de transporte satisfacen ciertos requerimientos. Esto se reporta en el Capítulo 3.

El modelo teórico se utilizó para el análisis de transporte de $\mathrm{NaCl}$ en diferentes sistemas de emulsión doble reportados en la literatura. Mediante ajustes de datos pertinentes de laboratorio, se reportaron los valores de la permeabilidad efectiva, difusión efectiva y del coeficiente de equilibrio en sistemas que utilizan combinaciones de aceite de canola, MCT o $\mathrm{R}(+)$-Limoneno como la fase continua, y gelatina y WPI/C63 como agentes estabilizadores. Se encontró que los sistemas con aceite de canola y gelatina presentan una liberación mucho más rápida de soluto en comparación con aquellos que utilizan MCTWPI. Esto es importante para el diseño adecuado de sistemas de membranas con liberación controlada.

Con los valores óptimos de los parámetros efectivos de transporte, se predijeron con el modelo teórico los perfiles de concentración de soluto dentro del sistema globular. En todos los casos se encontraron perfiles donde la concentración máxima se encuentra en el centro de las gotas. El tiempo en el que se alcanza el estado estacionario dentro de las gotas es el mismo cuando ya no existen cambios importantes en la liberación de soluto. 


\section{Referencias}

Becher, P. (1972). Emulsiones teoría y práctica. Madrid: Editorial Blume.

Betancourt-Grajales, R. (1991). Fenomenos de Transporte (1a ed.). Manizales: Universidad Nacional de Colombia.

Bhowal, A., y Datta, S. (1998). A mathematical model for the analysis of extraction data of weak acids/bases in liquid surfactant membranes. Journal Membrane Science, 139, 103-108.

Bhowal, A., y Datta, S. (2001). Studies on transport mechanism of Cr(VI) extraction from an acidic solution using liquid surfactant membranes. Journal Membrane Science, $188,1-8$.

Bird, R.B., Stewart, W.E., y Lightfoot, E.N. (1987). Fenómenos de Transporte.

Cárdenas, A. (1995). Membranas, generalidades. Universidad de los Andes, Mérida

Cortis, A., y Ghezzehei, T.A. (2007). On the transport of emulsions in porous media. Journal of Colloid Interface Science, 313, 1-4.

Chakraborty, M., Bhattacharya, C., y Datta, S. (2003). Effect of drop size distribution on mass transfer analysis of the extraction of nickel (II) by emulsion liquid membrane. Colloids and Surfaces A: Physicochem. Eng. Aspects, 224, 65-74.

Ching-Rong, H., Huifang, F., y Dingwei, Z. (2009). A closed-form solution for a mathematical model of emulsion liquid membrane. Journal Membrane Science, $339,233-238$.

Edelstein-Keshet, L. (1988). Mathematical Models in Biology. N. York.

El-Said, N., El-Sheref, E., y Borai, E. (2003). Modeling of transport of Cs(137) by emulsion liquid membrane(18C6) in xylene promoted by ephedrine hydrochloride in stripping phase. Journal Membrane Science, 211, 183-191.

González-Longatt, F.M. Métodos Numéricos para la solución de Ecuaciones Diferenciales: Un ejemplo comparativo.

Guoyu, M., Yuanli, J., y Chang, K.s. (1997). A general mass transfer model for liquid surfactant membranes. Chemical Engineering Journal, 52 (3), 433-441.

Huang, C.R., Fan, H., y Zhou, D. (2009). A closed-form solution for a mathematical model of emulsion liquid membrane. Journal Membrane Science, 339, 233-238. 
Huifang, F., Haiyun, Z., Hui, X., y Shilin, Q. (2012). Singularity in mathematical model of emulsion liquid membrane. Applied Mathematical Modelling, 36, 3736-3742.

Izquierdo-Gil, M.A., Barragán, V.M., Godino, M.P., Villaluenga, J.P.G., Ruiz-Bauzá, C., y Seoane, B. (2009). Salt diffusion through cation-exchange membranes in alcoholwater solutions. Separation and Purification Technology, 64, 321-325.

Kabalnov, A. (1994). Can micelles mediate a mass transfer between oil doplets? Langmuir, 680-684.

Kargari, K., Kaghazchi, T., y Soleimani, M. (2006). Mathematical modeling of emulsion liquid membrane pertraction of gold (III) from aqueous solutions. Journal Membrane Science, 279, 380-388.

Lanny, S., Muhammad, A.N., y Dérick, R. (2012). Stability and release properties of double emulsions for food applications. Food Hydrocolloids, 27, 316-323.

Lee, S.C., Chang, J.H., Ahn, B.S., y Lee, W.K. (1998). Mathematical modeling of Penicillin $\mathrm{G}$ extraction in an emulsion liquid membrane system containing only a surfactant in the membrane phase. Journal Membrane Science, 149, 39-49.

Li, N.N. (1968). US patent 3410794.

Liu, X., y Zhang, X. (1997). Simplified model for extraction of rare-earth ions using emulsion liquid membrane. Journal Membrane Science, 128, 223-229.

Luo, G.S., Chen, F., y Wang, Y.J. (2005). Mass transport and modeling of emulsion phaseinversion coating of hydrogel beads. Chemical Engineering Journal, 60, 1995-2003.

Lutz, R., Aserin, A., Wicker, L., y Garti, N. (2009). Release of electrolytes from W/O/W double emulsions stabilized by a soluble complex of modified pectin and whey protein isolate. Colloids and Surfaces B: Biointerfaces, 74, 178-185.

Morales-Zárate, E., Valdés-Parada, F.J., Goyeau, B., y Ochoa-Tapia, J.A. (2008). Diffusion and reaction in three-phase systems: Average transport equations and jump boundary conditions. Chemical Engineering Journal, 138, 307-332.

Murray, J.D. (1991). Mathematical Biology (second edition ed.). Springer, N. York.

Muschiolik, G. (2007). Multiple emulsions for food use. Current Opinion in Colloid \& Interface Science, 12, 213-220.

Okubo, A. (1980). Diffusion and Ecological Problems: Mathematical Models. N. York. 
Ôzer, Ô., Muguet, V., Roy, E., Grossiord, J.L., y Seiller, M. (2000). Stability study of W/O/W viscosified multiple emulsions. Drug Development and Industrial Pharmacy, 26 (11), 1185-1189.

Pérez-Orozco, J.P., Barrios-Salgado, E., Róman-Guerrero, A., y Pedroza-Islas, R. (2011). Interacción goma de mezquite-quitosano en la interfase y su influencia en la estabilidad de emulsiones múltiples W1/O/W2 Revista Mexicana de Ingeniería Química, 10 (3), 487-499.

Piotr, D., Benoit, A., Sybrand, J.M., Kitty, N., y Matthias, W. (2010). Transport limitations in ion exchange membranes at low salt concentrations. Journal Membrane Science, $346,163-171$.

Ramírez, J.J., Vanegas, C.A., y Villegas, A.M. (2005). Método de diferencias Finitas y Elemento Finito para la Solución de Ecuaciones Estocásticas.

Raynal, S., Grossiord, J.L., Seiller, M., y Clausse, D. (1993). J. Control. Release, 26, 129.

Reis, M.T.A., y Carvalho, J.M.R. (2004). Modeling of zinc extraction from sulphate solutions with bis(2-ethylhexyl)thiophosphoric acid by emulsion liquid membranes. Journal Membrane Science, 97-107.

Salager, J.L. (1999). Formulación, composición y Fabricación de Emulsiones para obtener las Propiedades deseadas. Universidad de los Andes, Mérida

Salvadori, y Baron. (1992). Numerical Methods in Engineering.

Sapei, L., Ali Naqvi, M., y Rousseau, D. (2012). Stability and release properties of double emulsions for food applications. Food Hydrocolloids, 27, 316-323.

Sata, T. (2004). Ion Exchange Membranes, Edition. The Royal Society of Chemistry Cambridge.

Seifritz, W. (1925). Study of emulsions. J. Phys. Chem., 29, 738-748.

Simal, S., Sánchez, E.S., Bon, J., Femenia, A., y Rosselló, C. (2001). Water and salt di usion during cheese ripening: e ect of the external and internal resistances to mass transfer. Journal of Food Engineering, 48, 269-275.

Smith, G. (1999). Numerical Solution of Partial Differential Equations: Finite Difference Methods. Oxford University Press, New York.

Soo, H. (1986). A filltration model for the flow of dilute, stable emulsions in porous mediaI. Theory. Chemical Engineering Science, 41(2), 263-272. 
Strathmann, H. (2004). Ion-Exchange Membrane Separation Processes. Membrane Science and Technology, 9, First Edition, Elserver.

Wood, B.D., y Whitaker, S. (2000). Multi-species diffusion and reaction in biofilms and celular media. Chemical Engineering Science, 55, 3397-3418.

Wronski, S., Vladimirov, V., y Adach, A. (2012). Modelling of mass transfer from multiple emulsions. International Journal of Heat and Mass Transfer, 55, 4241-4245.

Yan, N., Shi, Y., y Su, Y.F. (1992). A mass transfer model for type I facilitated transport in liquid membranes. Chemical Engineering Science, 47(17/18), 4365-4371.

Yunus, C., y Afshin, G. (2011). Transferencia de calor y masa (4 ed.): McGraw-Hill. 


\section{Anexo A}

\section{Código de Matlab}

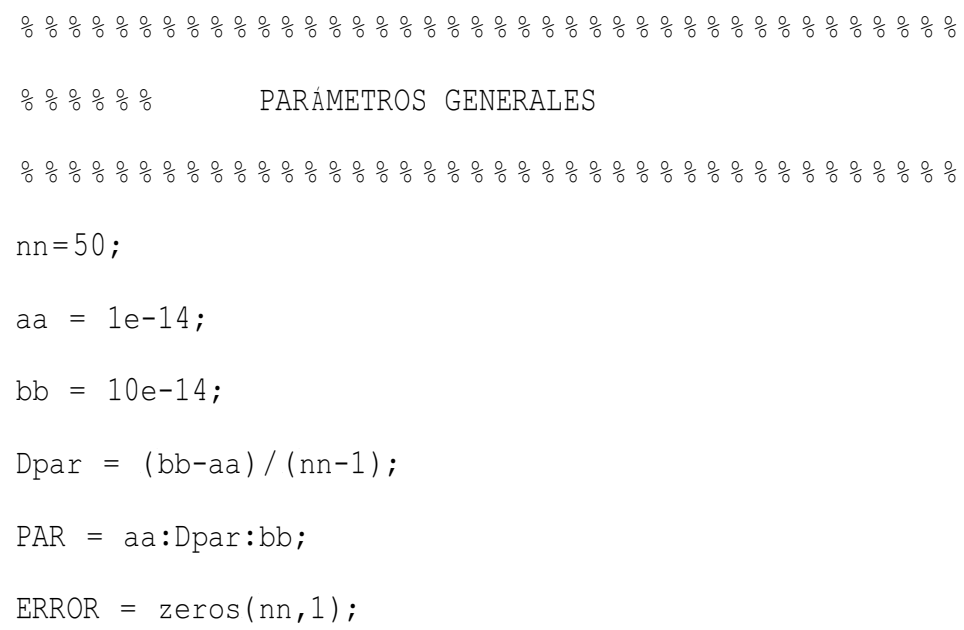

Emw $=0.6776 ;$ ofracción volumétrica; MOVER

CondIni $=0.924 ; \%$ Condición inicial, MOVER

Factor $=100 * 80 /$ CondIni; $\%$ Factor que multiplica a $\mathrm{Cw}$ para obtener la misma variable del eje y que en los datos experimentale, MOVER

Tsimulacion $=800 ; \%$ horas MOVER

$\mathrm{R}=1.85 \mathrm{e}-4 ;$ \% radio de las gotas grandes, $\mathrm{m} ;$ MOVER

$\mathrm{T}=\mathrm{Tsimulacion} * 3600 ;$ otiempo de simulación

$\mathrm{n} \_\mathrm{r}=50 ;$ onumero de puntos en la coordenada radial 
$n_{-} t=700 * n_{-} r ; \%$ numero de puntos en el tiempo

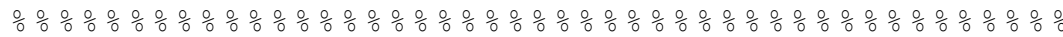

음 PARÁMETROS DEL MODELO DE MORALES-ZÁRATE ET AL. (2009)

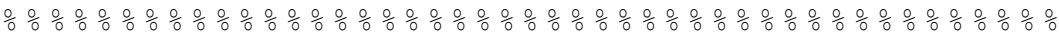

Erw = 1 - Emw; ofracción volumétrica

$\mathrm{av}=1 ;$ ónea interfacial volumétrica de gota $\left(\mathrm{m}^{\wedge}-1\right)$;fija

Peff $=\mathrm{P} *$ Keq $;$ o permeabilidad efectiva $(\mathrm{m} / \mathrm{s})$ fija

Keff $=1 ;$ o coeficiente de equilibrio efectivo; fija

$\div \operatorname{Vn}=1 ;$ volumen de la fase eta contenida en el volumen promedio $\left(\mathrm{m}^{\wedge} 3\right) ;$ fija

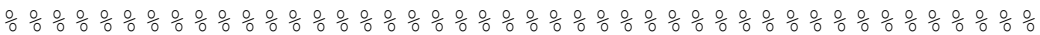

응 datos articulos

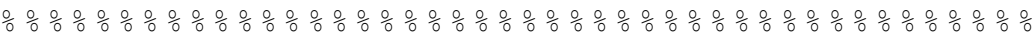

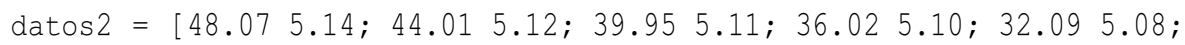
$28.035 .06 ; 24.035 .03 ; 20.174 .98 ; 15.984 .88 ; 11.984 .73 ;$

$8.064 .48 ; 5.994 .31 ; 3.994 .08 ; 3.993 .89 ; 3.06 \quad 3.61 ;$

$2.063 .39 ; 1.53 \quad 3.14 ; 1.132 .97 ; 0.802 .77 ; 0.53 \quad 2.61] ;$

datos $4=\left[\begin{array}{lll}48.005 .04 ; 44.015 .04 ; 39.95 & 5.03 ; 36.025 .02 ; 32.094 .99 ;\end{array}\right.$

$28.034 .96 ; 24.104 .93 ; 20.044 .89 ; 15.914 .82 ; 11.984 .70 ;$

$7.994 .49 ; 5.99 \quad 4.31 ; 2.934 .08 ; 2.13 \quad 3.89 ; 2.00 \quad 3.60 ;$

$1.533 .49 ; 1.40 \quad 3.28 ; 1.132 .97 ; 0.472 .75 ; 0.53 \quad 2.61]$;

datos $6=[48.074 .92 ; 44.014 .92 ; 39.954 .91 ; 36.024 .89 ; 32.164 .86 ;$

$27.964 .84 ; 24.034 .82 ; 20.044 .76 ; 16.054 .71 ; 12.124 .61 ;$

$7.994 .44 ; 6.06 \quad 4.31 ; 3.934 .07 ; 3.06 \quad 3.89 ; 2.00 \quad 3.59 ;$

$1.403 .37 ; 1.003 .13 ; 0.872 .96 ; 0.472 .81 ; 0.47 \quad 2.60] ;$

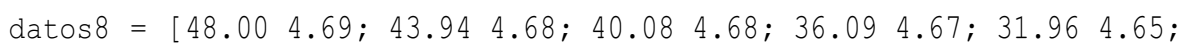

$28.034 .63 ; 24.034 .60 ; 20.044 .57 ; 15.984 .52 ; 12.054 .46 ;$

$8.064 .33 ; 6.064 .21 ; 4.064 .01 ; 2.863 .84 ; 2.06 \quad 3.61 ;$

$1.463 .42 ; 1.003 .18 ; 0.803 .04 ; 0.532 .89 ; 0.47 \quad 2.60] ;$ 


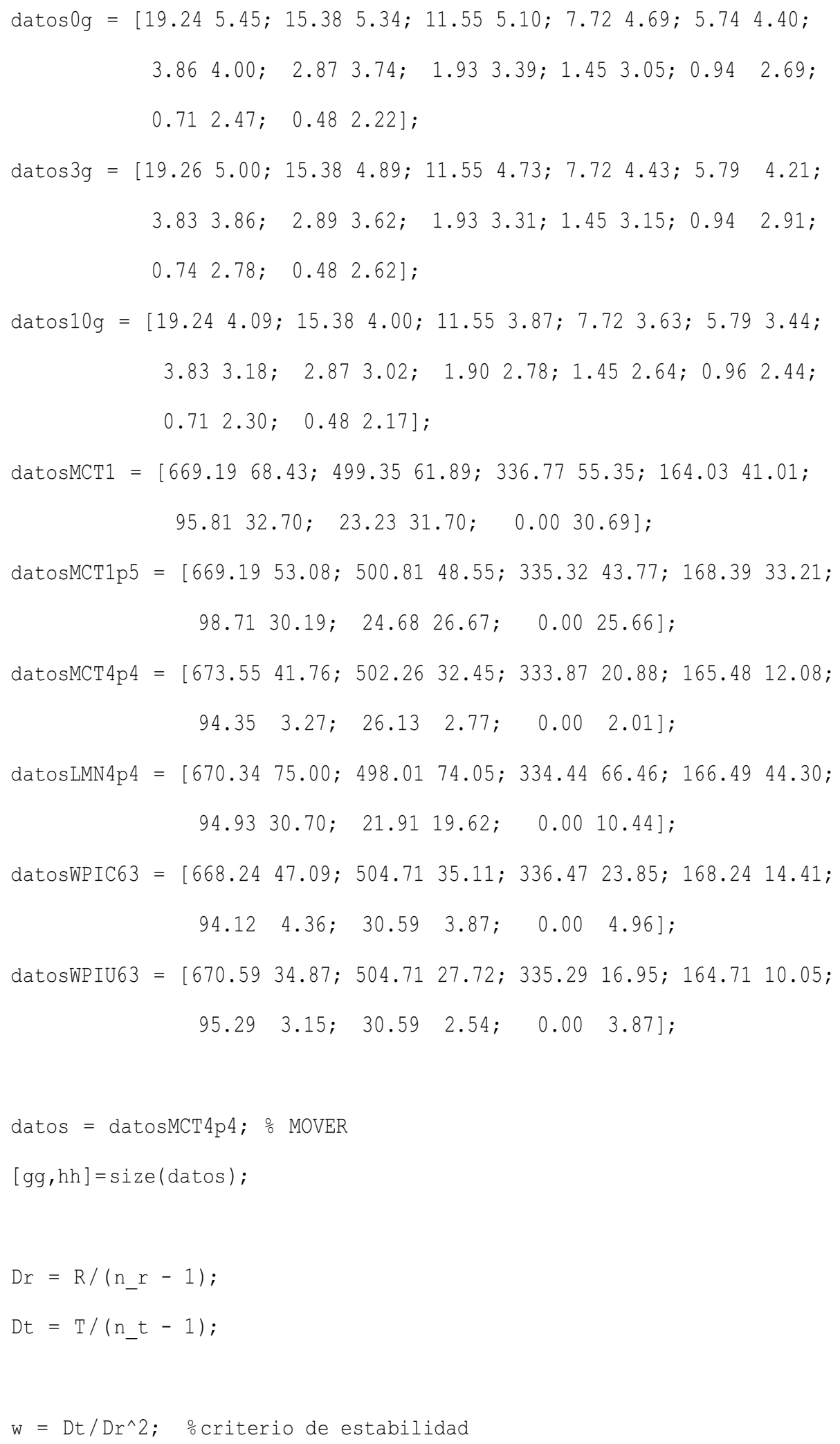


$C s(k+1)=C s(k)+k l a * D t *(C(i, k)-C s(k)) ;$

end

$\mathrm{CW}_{\mathrm{w}}(1, \mathrm{k}+1)=\mathrm{CW}_{\mathrm{w}}(2, \mathrm{k}+1)$; $\frac{\mathrm{o}}{\mathrm{a}} \mathrm{actualización} \mathrm{de} \mathrm{condición} \mathrm{de} \mathrm{frontera} \mathrm{en} \mathrm{r}=0$

$C(1, k+1)=C(2, k+1) ; \quad \frac{o}{0}$ condición de frontera en $r=0$

$\mathrm{CW}_{\mathrm{w}}\left(\mathrm{n} \_\mathrm{r}, \mathrm{k}+1\right)=\left(\mathrm{Cw}\left(\mathrm{n} \_-1, \mathrm{k}+1\right)+\mathrm{S}{ }^{*} \mathrm{Ch}(\mathrm{k}+1)\right) /(1+\mathrm{S}$ Keff $)$; \%actualización de condición de frontera en $r=R$ en $r=R$

$C\left(n \_r, k+1\right)=C\left(n \_r-1, k+1\right) *(1-h * D r / D a)$; octualización condición de frontera

$C h(k+1)=C h(k)-Z^{*}\left(C h(k)-\operatorname{Keff*Cw}\left(n \_r, k\right)\right) ;$

end

$[$ ndatos, $q$ q $]=\operatorname{size}($ datos $)$;

postime $=$ zeros $($ ndatos, 1$)$;

for $j=1$

for $i=n_{-} t:-1: 1$

if $t(i) / 3600<\operatorname{datos}(j, 1)$

postime $(j)=i$;

break

end

end

end

for $j=2$ :ndatos

for $i=\operatorname{postime}(j-1):-1: 1$

if $t(i) / 3600<\operatorname{datos}(j, 1)$

postime $(j)=i ;$ 


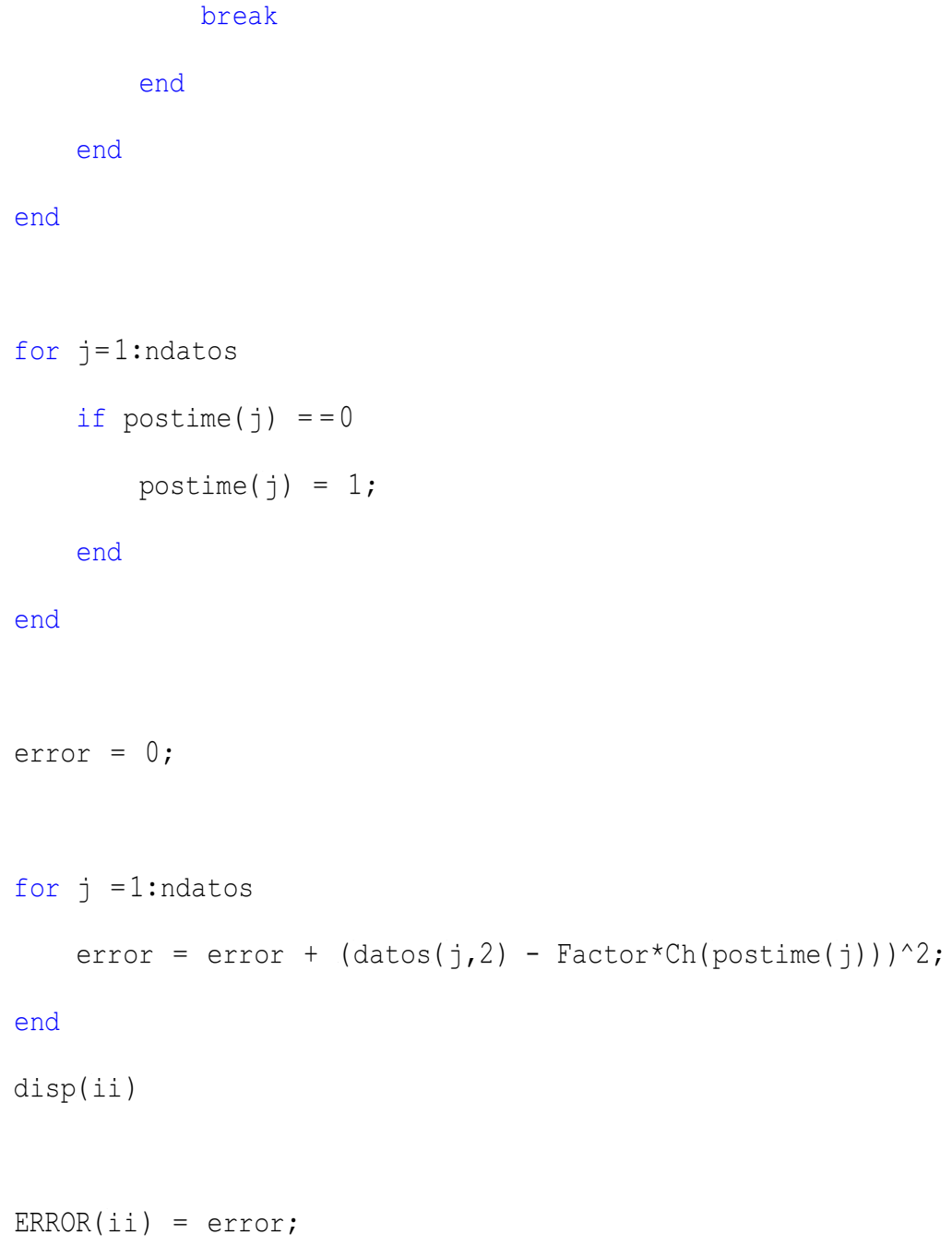


Anexos

optimo $=\operatorname{PAR}(\mathrm{pp} 2) ;$ 


\section{Anexo B}

\section{Modelado de la transferencia de masa en la microescala}

En este anexo se muestran las ecuaciones gobernantes para el modelo en la microescala, las cuales fueron resueltas utilizando COMSOL. En principio, es posible resolver las ecuaciones utilizando un modelo con geometría en 3D (ver Figura 27); sin embargo, los esfuerzos de computo se incrementan notablemente por lo que se decidió resolver un modelo en 2D con geometría más sencilla. En la Figura 27 se observa la geometría en 3D del modelo propuesto. En el lado a) de la figura se muestra la gota externa de la emulsión doble y en el lado b) las gotas internas de dicha emulsión.
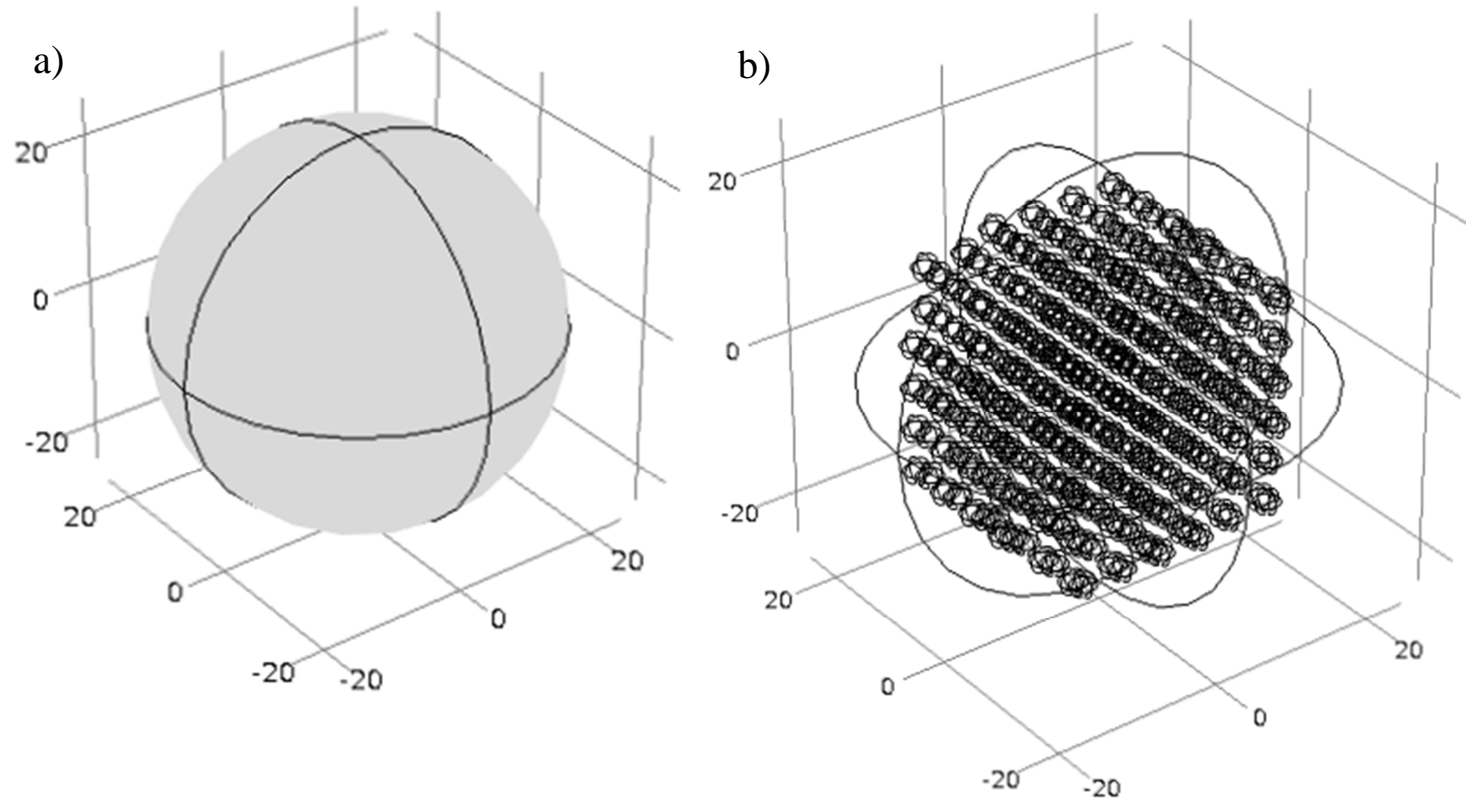

Figura 27. Geometría en 3D para un sistema de emulsión doble.

Las ecuaciones utilizadas para el modelo en la microescala se retoman del trabajo de Morales-Zárate et al., (2008) y se plantean a continuación.

Las ecuaciones locales que gobiernan el transporte de masa difusivo son: 
Para la fase $\sigma$ :

$$
\frac{\partial C_{\sigma}}{\partial t}=\nabla \cdot\left(D_{\sigma} \nabla C_{\sigma}\right)
$$

Para la fase $\mu$ :

$$
\frac{\partial C_{\mu}}{\partial t}=\nabla \cdot\left(D_{\mu} \nabla C_{\mu}\right)
$$

Para la fase $\gamma$ :

$$
\frac{\partial C_{\gamma}}{\partial t}=\nabla \cdot\left(D_{\gamma} \nabla C_{\gamma}\right)
$$

Aquí cada una de las fases se identifican en la Figura 5. Las ecuaciones (95)-(97) están asociadas a las siguientes condiciones de frontera interfaciales.

En la interface $\sigma \mu$ :

$$
\begin{gathered}
-\mathbf{n}_{\mu \sigma} \cdot D_{\mu} \nabla C_{\mu}=-\mathbf{n}_{\mu \sigma} \cdot D_{\sigma} \nabla C_{\sigma} \\
-\mathbf{n}_{\sigma \mu} \cdot D_{\sigma} \nabla C_{\sigma}=P_{\sigma \mu}\left(C_{\sigma}-K_{e q}^{\mu \sigma} C_{\mu}\right)
\end{gathered}
$$

En la interface $\mu \gamma$ :

$$
\begin{gathered}
-\mathbf{n}_{\mu \gamma} \cdot D_{\mu} \nabla C_{\mu}=-\mathbf{n}_{\mu \gamma} \cdot D_{\gamma} \nabla C_{\gamma} \\
-\mathbf{n}_{\mu \mu} \cdot D_{\gamma} \nabla C_{\gamma}=P_{\gamma \mu}\left(C_{\gamma}-K_{e q}^{\mu \gamma} C_{\mu}\right)
\end{gathered}
$$

Las ecuaciones (98)-(101) han sido derivadas siguiendo a Wood y Whitaker (2000).

Dónde:

$P_{\sigma \mu}, P_{\gamma \mu}, K_{e q}^{\mu \sigma}$ y $K_{e q}^{\mu \gamma}$ son funciones de las constantes de equilibrio locales. En las ecuaciones anteriores $\mathbf{n}_{\mu \sigma}$ representa el vector normal unitario dirigido desde la fase $\mu$ hacia la fase $\sigma$ 
$K_{e q}^{\mu \sigma}$ y $K_{e q}^{\mu \gamma}$ son los coeficientes de distribución de equilibrio, mientras que la permeabilidad de la membrana de las interfases $\sigma \mu$ y $\gamma \mu$ están dadas por $P_{\sigma \mu}$ y $P_{\gamma \mu}$, respectivamente.

Los valores utilizados en el modelo en la microescala se reportan a continuación:

Para la fase acuosa:

$D_{\sigma}$ y $D_{\gamma}=5 \times 10^{-15} \mathrm{~m}^{2} / \mathrm{s}$

Para la fase oleosa:

$D_{\mu}=1 \times 10^{-15} \mathrm{~m}^{2} / \mathrm{s}$

Para la permeabilidad:

$P_{\sigma \mu}$ у $P_{\gamma \mu}=1 \mathrm{~m} / \mathrm{s}$

Para la constante de equilibrio:

$K_{e q}^{\mu \sigma}$ y $K_{e q}^{\mu \gamma}=1$

La geometría bidimensional utilizada para resolver las ecuaciones anteriores se muestra en la Figura 28. Donde se observa la gota exterior y las gotas interiores. 


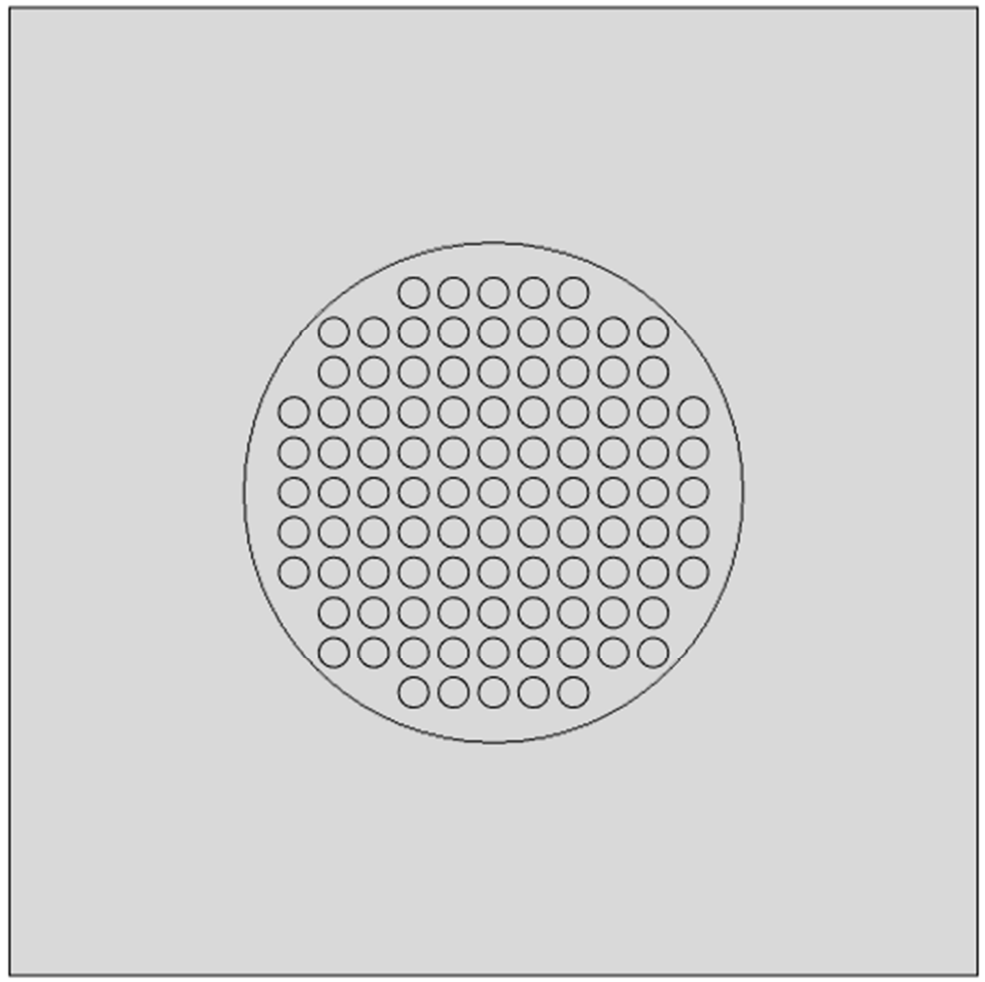

Figura 28. Geometría en 2D del modelo propuesto.

La Figura 29 muestra la liberación del soluto para diferentes tiempos. Para el tiempo cero se observa que todo el soluto se encuentra en las gotas internas, al paso de una hora el soluto empieza a salir hacia el exterior, al llegar a las 9 horas gran parte del soluto se encuentra en las gotas exteriores, cuando el experimento llega a la etapa final, es decir las 50 horas de monitoreo en las gotas internas ya no es posible observar el soluto. Lo que se puede resumir de la figura es que la liberación del soluto se ha dado en una forma rápida. 
\ 0.1207

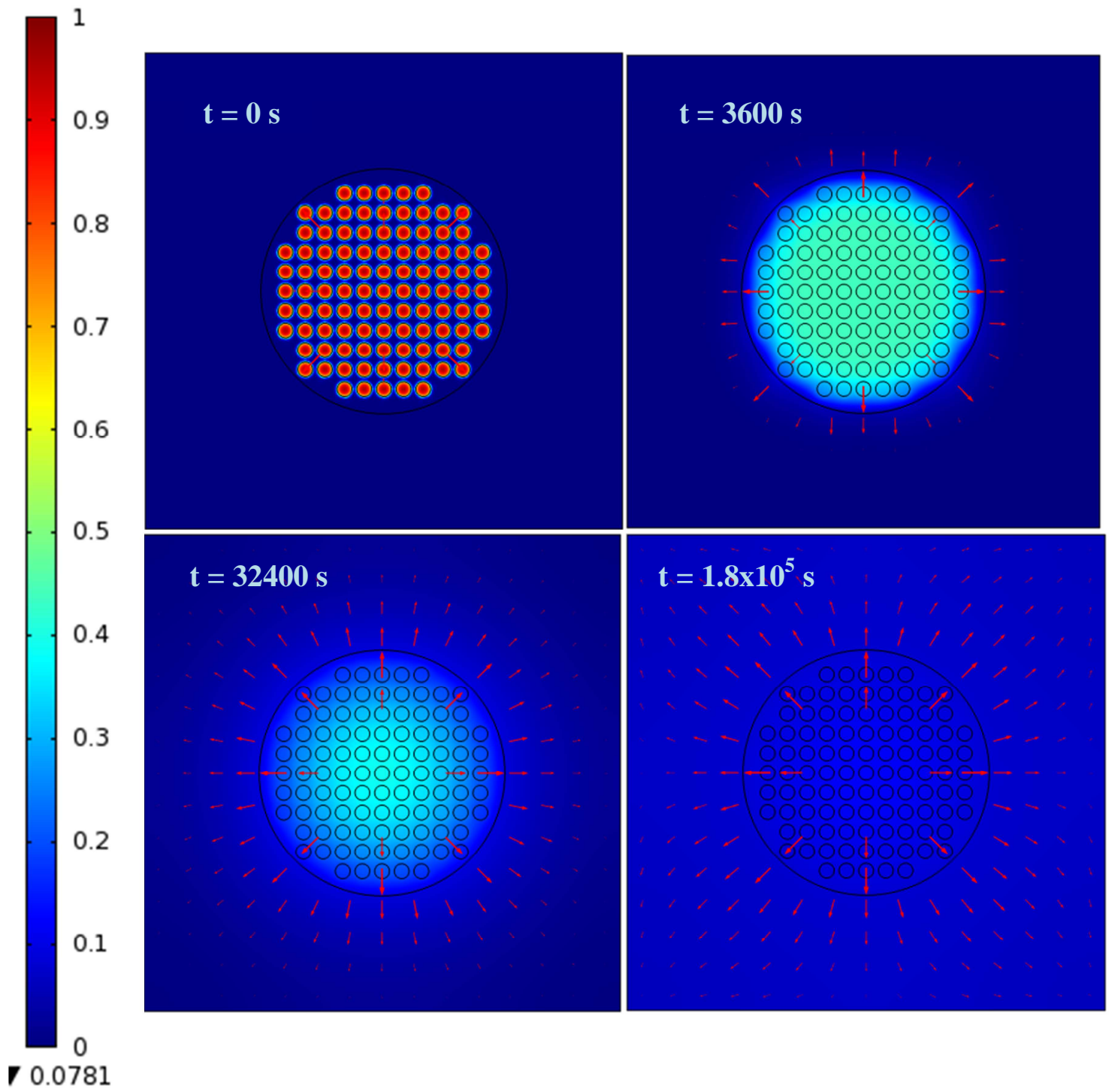

Figura 29. Esquema de liberación de soluto para diferentes tiempos.

La Figura 30 presenta los perfiles de liberación en la dirección radial. Para medir la concentración en este caso, la geometría se dividió a la mitad por una línea recta. La forma oscilatoria de la figura se debe a que en el arco de la onda superior el soluto se encuentra aún en la gota interior. Es decir, que la liberación del soluto se calculando en la gota 
interior que se encuentra dentro de la gota exterior, y el arco en la parte inferior indica que la liberación se está evaluando fuera de las gotas interiores pero dentro de la gota exterior.

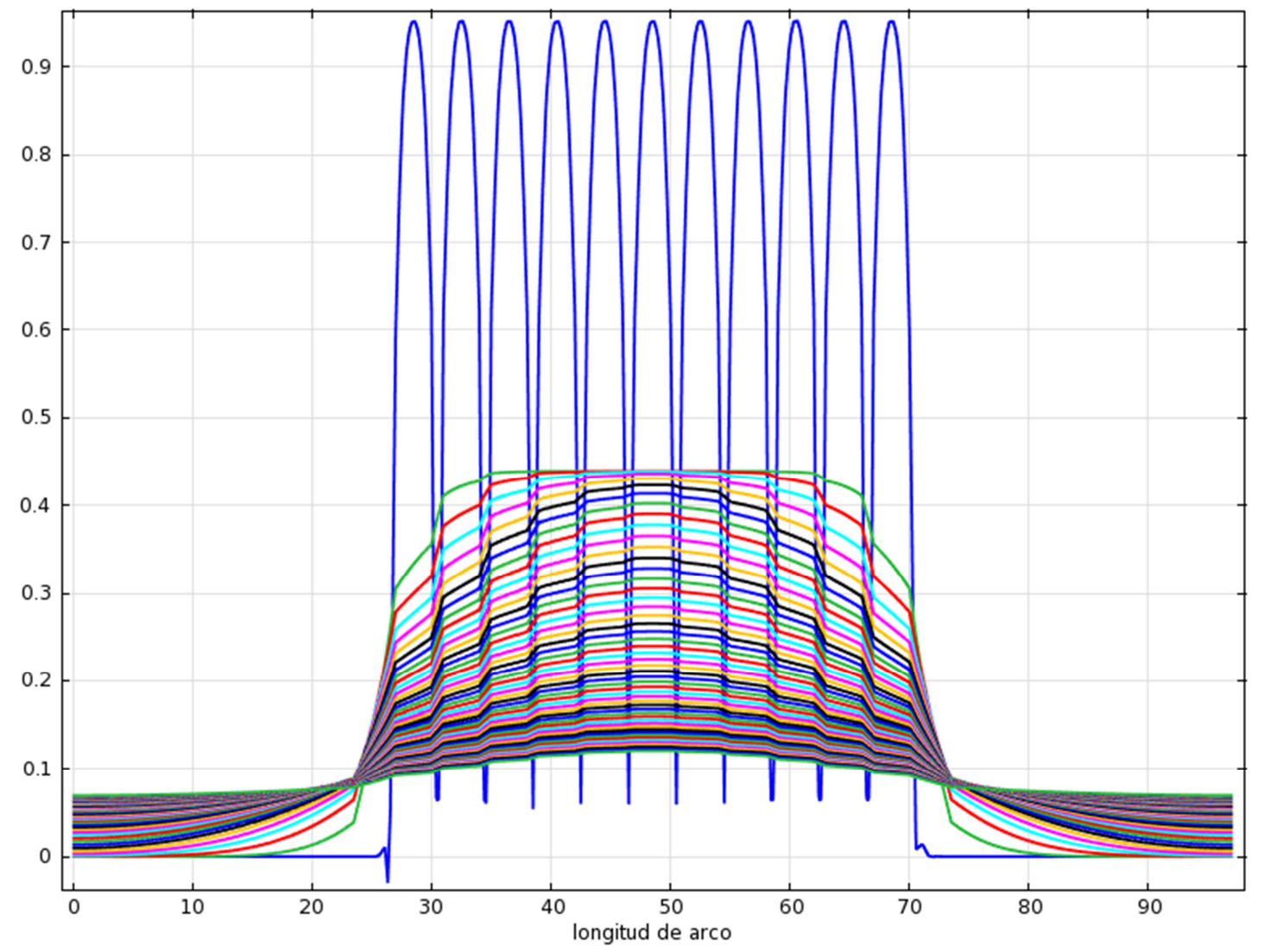

Figura 30. Perfiles de concentración en la dirección radial.

El perfil de liberación de soluto se observa en la Figura 31, con un porcentaje de liberación de soluto de aproximadamente 0.07 , en la figura no se observa un cambio significativo pero se aprecia que el estado estacionario se alcanzara en un tiempo mayor al que se le dio al realizar los experimentos (50 horas). La Figura 31 coincide con lo dicho en la Figura 29. 


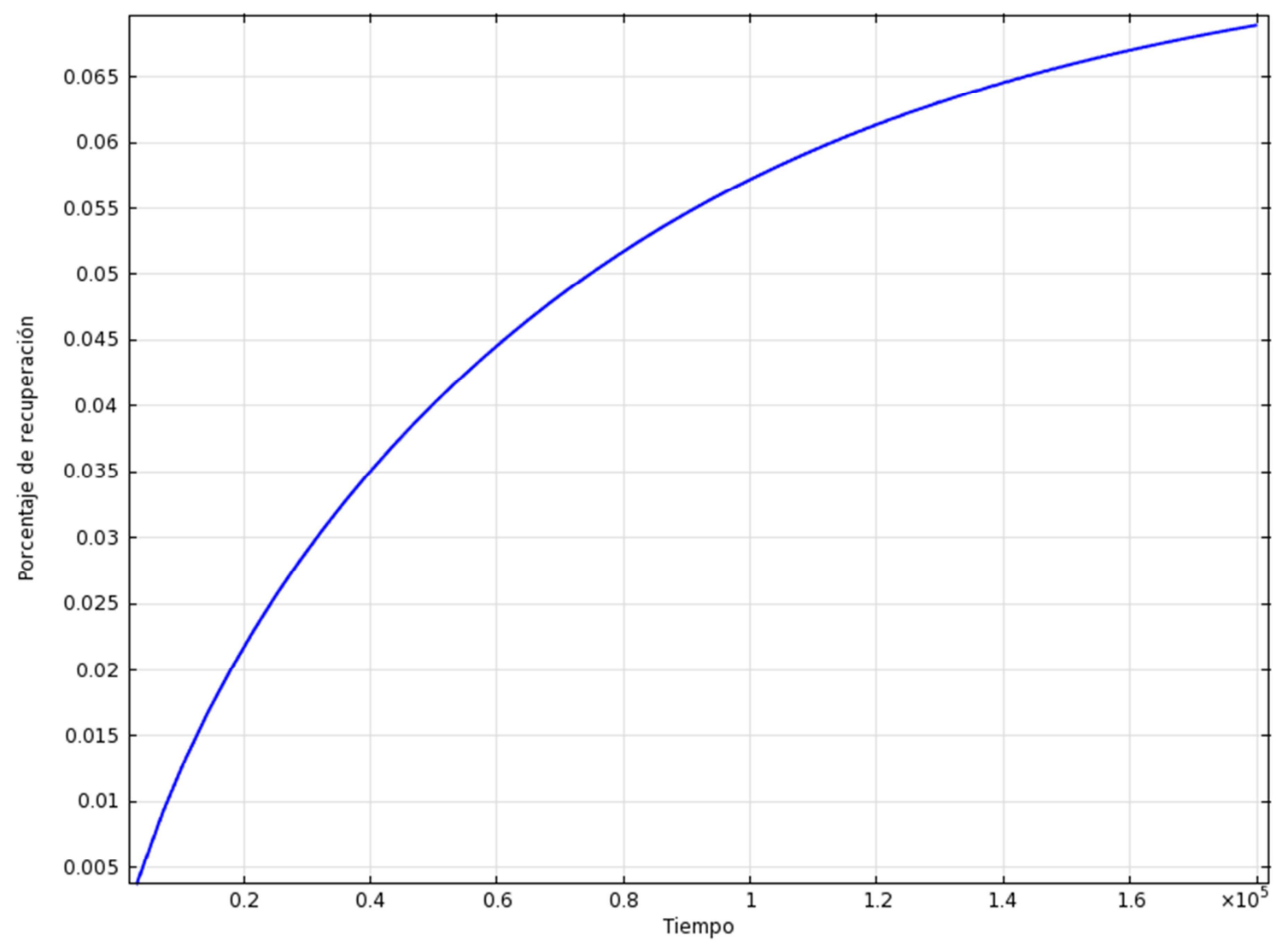

Figura 31. Perfil de liberación de soluto 
